\title{
THERMOPHYSICAL PROPERTIES OF PENETRANTS
}

IN POLYMERS VIA A PIEZOELECTRIC QUARTZ

CRYSTAL MICROBALANCE

\author{
By \\ SIVA PRASAD MIKKILINENI \\ Bachelor of Engineering \\ and \\ Master of Science \\ Birla Institute of Technology and Science \\ Pilani, India \\ 1992 \\ Submitted to the Faculty of the \\ Graduate College of the \\ Oklahoma State University \\ in partial fulfillment of \\ the requirements for \\ the Degree of \\ MASTER OF SCIENCE \\ December, 1995
}




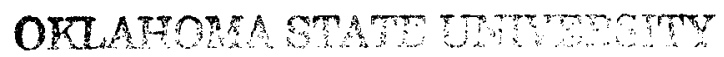

THERMOPHYSICAL PROPERTIES OF PENETRANTS IN POLYMERS VIA A PIEZOELECTRIC QUARTZ CRYSTAL MICROBALANCE

Thesis Approved:

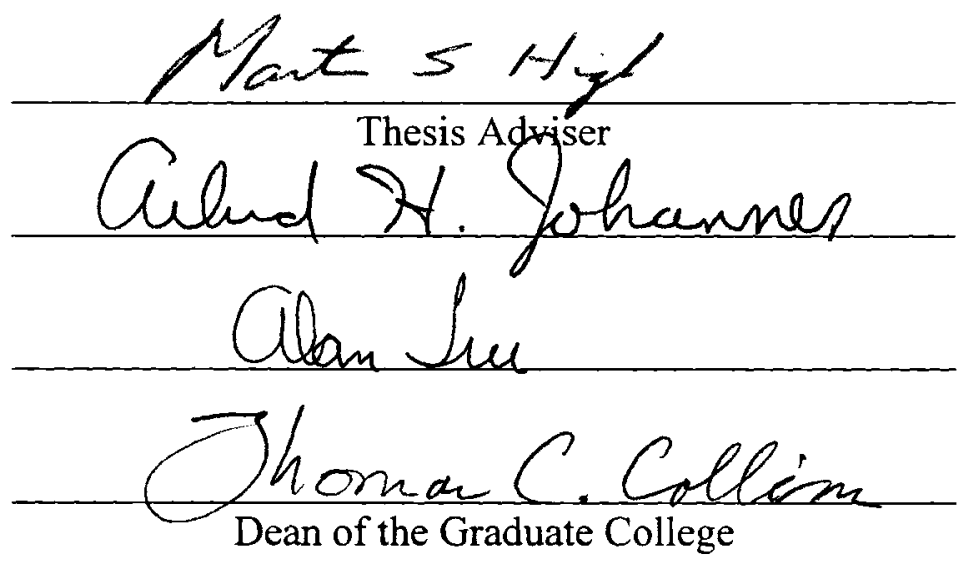

ii 


\section{ACKNOWLEDGMENTS}

I would like to express my appreciation to my advisor, Dr. Martin S. High, for his sincere, prompt, and persistent intellectual and financial support on this project. I would also thank him for being so approachable and patient. I would also like to thank Dr. Alan Tree for his guidance and suggestions.

I am greatly indebted to my family for having supported me emotionally. Lastly, I would like to thank my roommates and friends at Stillwater for their company, friendship and support.

Financial support from Rexham Graphics Inc. and Dow Chemical Company is greatly appreciated. 


\section{TABLE OF CONTENTS}

Chapter Page

I. INTRODUCTION .1

II. LITERATURE REVIEW

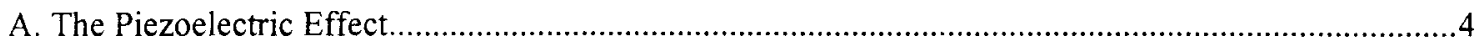

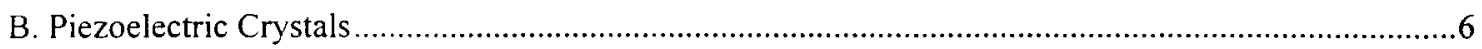

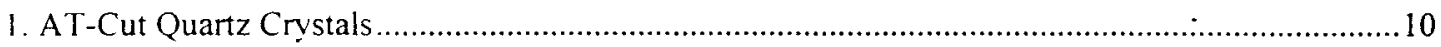

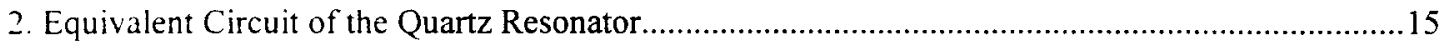

3 The Temperature Coefficient of Frequency for Quartz Crystals ..................................................18

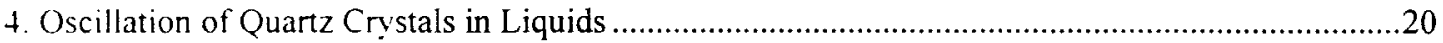

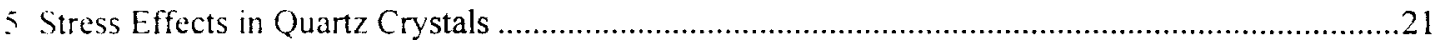

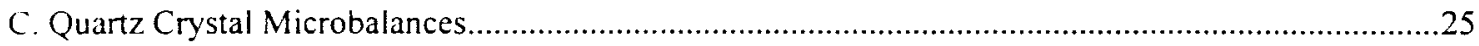

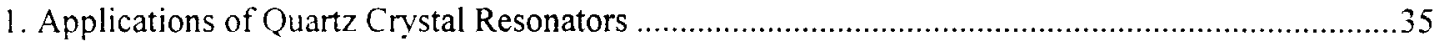

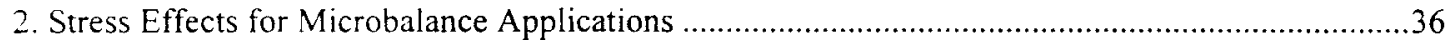

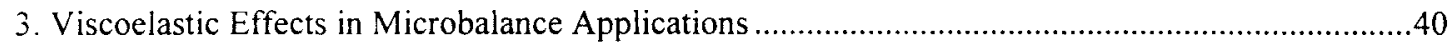

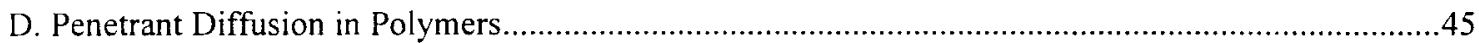

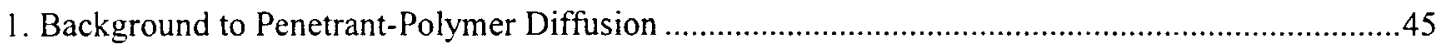

2. Classical Experimental Methods for Penetrant-Polymer Diffusion................................................50

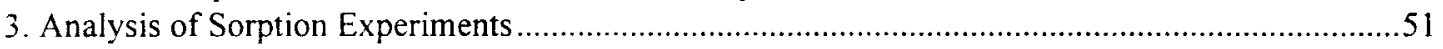

4. Free Volume Model for Polymer-Penetrant Diffusionon ......................................................5

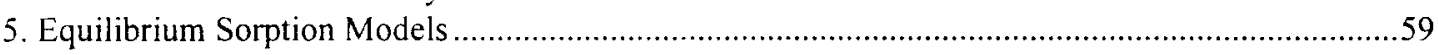

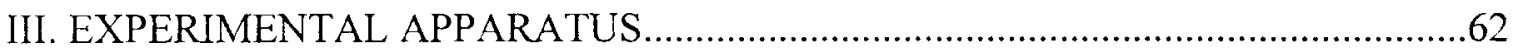

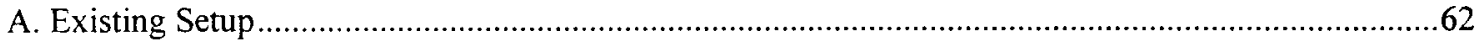

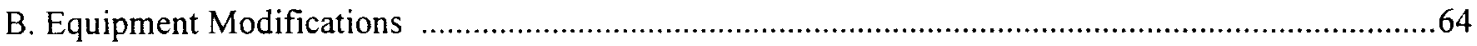

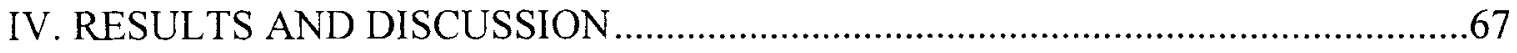

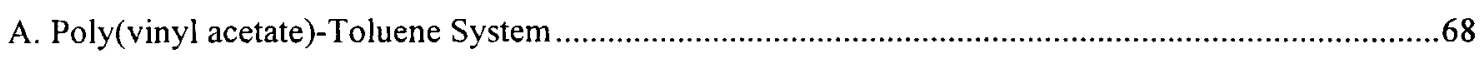

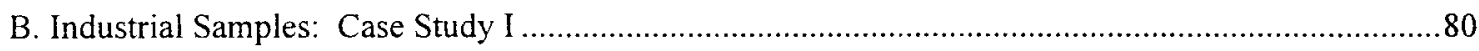




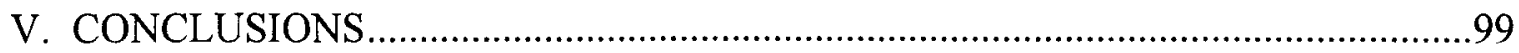

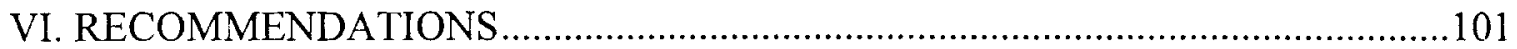

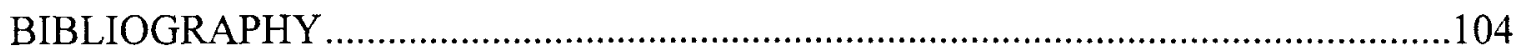

APPENDIX A: OPERATING INSTRUCTIONS FOR THE QUARTZ CRYSTAL

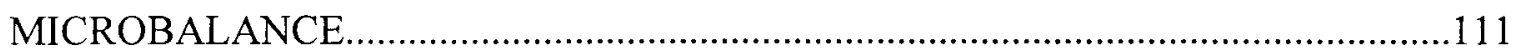

APPENDIX B: ERROR AND SENSITIVITY ANALYSIS ......................................... 


\section{LIST OF TABLES}

Table Page

I. Applications of Quartz Crystals 37

II. Equilibrium Sorption of Toluene and Poly(vinyl acetate) Measured via the Quartz Crystal Microbalance

III. Equilibrium Sorption of Toluene in the 8883 Sample Measured via the Quartz Crystal Microbalance 82

IV. Equilibrium Sorption of Toluene in the 8899 Sample Measured via the Quartz Crystal Microbalance

V. Sensitivity Analysis for Frequency Changes 124

VI. Sensitivity Aalysis for Canges in Tickness and Slope of the Sorption Curve. 


\section{LIST OF FIGURES}

Figure

1. Angle of Cut and Orientation of an AT-cut Quartz Crystal...........................................11

2. Equivalent Circuits of Electromagnetic, Electromechanical and

Electrostatic Transducers .................................................................................16

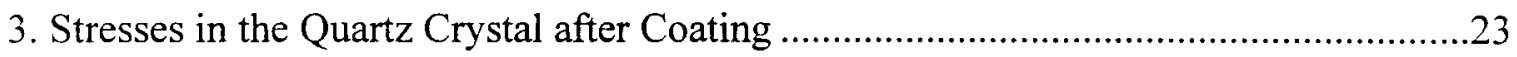

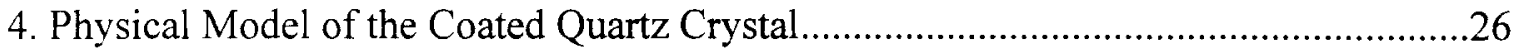

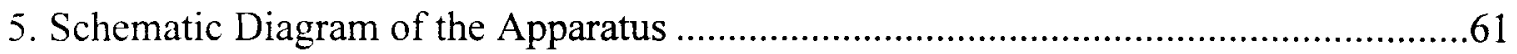

6. Temperature Dependence of the Frequency of the Bare and

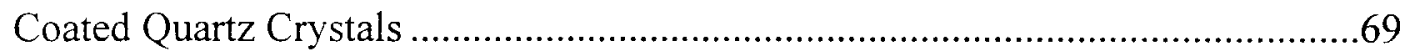

7. Mutual Diffusivity of Toluene and Poly(vinyl acetate) at $40^{\circ} \mathrm{C}$ for the Quartz Spring Balance (Ju, 1981), Quartz Crystal Microbalance and

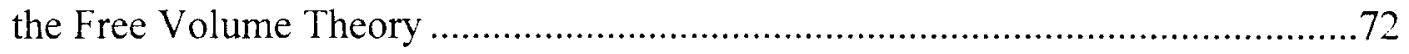

8. Equilibrium Sorption Experiments of Toluene and Poly(vinyl acetate) at $40^{\circ} \mathrm{C}$..........73

9. Mutual Diffusivity of Toluene and Poly(vinyl acetate) at $60{ }^{\circ} \mathrm{C}$ for Quartz Spring Balance (Hou, 1986), Quartz Crystal Microbalance and the Free Volume Theory

10. Equilibrium Sorption Experiments of Toluene and Poly(vinyl acetate) at $60^{\circ} \mathrm{C}$........75

11. Mutual Diffusivity of Toluene and Poly(vinyl acetate) at $80{ }^{\circ} \mathrm{C}$ for the Quartz Spring Balance (Hou, 1986), Quartz Crystal Microbalance and the Free Volume Theory

12. Equilibrium Sorption Experiments of Toluene and Poly(vinyl acetate) at $80^{\circ} \mathrm{C}$.........77

13. Mutual Diffusivity of Toluene and 8883 Sample at $60{ }^{\circ} \mathrm{C}$ for the Quartz Crystal Microbalance 
14. Mutual Diffusivity of Toluene and 8883 Sample at $80{ }^{\circ} \mathrm{C}$ for the Quartz Crystal Microbalance.

15. Mutual Diffusivity of Toluene and 8899 Sample at $60{ }^{\circ} \mathrm{C}$ for the Quartz Crystal Microbalance.

16. Mutual Diffusivity of Toluene and 8899 Sample at $80{ }^{\circ} \mathrm{C}$ for the Quartz Crystal Microbalance

17. Equilibrium Sorption Experiments of Toluene and 8883 Sample at $60{ }^{\circ} \mathrm{C}$

18. Equilibrium Sorption Experiments of Toluene and 8883 Sample at $80^{\circ} \mathrm{C}$ .90

19. Equilibrium Sorption Experiments of Toluene and 8899 Sample at $60^{\circ} \mathrm{C}$. 91

20. Equilibrium Sorption Experiments of Toluene and 8899 Sample at $80{ }^{\circ} \mathrm{C}$ .92

21. Frequency vs Time During Desorption of the Polymer Sample in order to Determine the Frequency of the Polymer and the Crystal .94

22. Frequency vs Time after the Introduction of Solvent into the Chamber .95

23. Sensitivity of Frequencies in the Calculation of Diffusion Coefficient 126 


\section{NOMENCLATURE}

A piezoelectrically active area of the quartz crystal (nm)

$\mathrm{A}_{\mathrm{o}}$ non-damped amplitude of the quartz crystal (nm)

$\Delta \mathrm{A}$ amplitude change of the quartz crystal (nm)

c elastic constant shear stiffness constant in the coated film shear stiffness constant in the quartz crystal capacitance $(\mathrm{pF})$

$\mathrm{C}_{0}$ shunt capacitance $(\mathrm{pF})$

$\mathrm{C}_{\mathrm{f}}$ mass sensitivity of the quartz crystal

$\mathrm{c}_{\mathrm{ij}}$ ratio of stress to strain associated with the elastic wave propagated in the quartz

$\mathrm{df}_{\mathrm{s}}$ frequency change due to stress effects $(\mathrm{Hz})$

D mutual diffusivity $\left(\mathrm{cm}^{2} / \mathrm{s}\right)$

$\mathrm{D}^{*} \quad$ mutual diffusion coefficient $\left(\mathrm{cm}^{2} / \mathrm{s}\right)$

$\mathrm{D}_{1} \quad$ self diffusion coefficient of the solvent $\left(\mathrm{cm}^{2} / \mathrm{s}\right)$

$\mathrm{D}_{2} \quad$ self diffusion coefficient of the polymer $\left(\mathrm{cm}^{2} / \mathrm{s}\right)$

DEB diffusion deborah number

e thickness of the plate $(\mathrm{cm})$

$\mathrm{f}_{\mathrm{o}} \quad$ bare crystal frequency $(\mathrm{Hz})$ 
$f_{s}$

$f_{p}$

$f_{c}$

$f_{\mathrm{f}}$

$\Delta \mathrm{f}$

$G(t)$

$\mathrm{h}$

I

$\mathrm{k}$

K

L

$\mathrm{m}_{\mathrm{q}}$

$\mathrm{m}_{\mathrm{f}}$

$M_{f}$

$\mathrm{M}_{\mathrm{q}}$

$\Delta \mathrm{m}$

QCM quartz crystal microbalance

r

$\mathrm{R}$

moment of inertia

dielectric constant

inductance $(\mathrm{H})$

mass of the coated film

mass of the quartz crystal

mass change

damping coefficient

resistance $(\mathrm{Ohm})$ series resonant frequency $(\mathrm{Hz})$

parallel resonant frequency $(\mathrm{Hz})$

frequency of the quartz film compound resonator $(\mathrm{Hz})$

frequency of the film alone if the film were oscillating $(\mathrm{Hz})$

measured frequency shift $(\mathrm{Hz})$

shear relaxation modulus of the system

stiffness constant of the hair spring

constant dependent on the crystallographic orientation of the crystal

areal density of the quartz crystal $\left(\mathrm{cm}^{2}\right)$

areal density of the coated film $\left(\mathrm{cm}^{2}\right)$ 


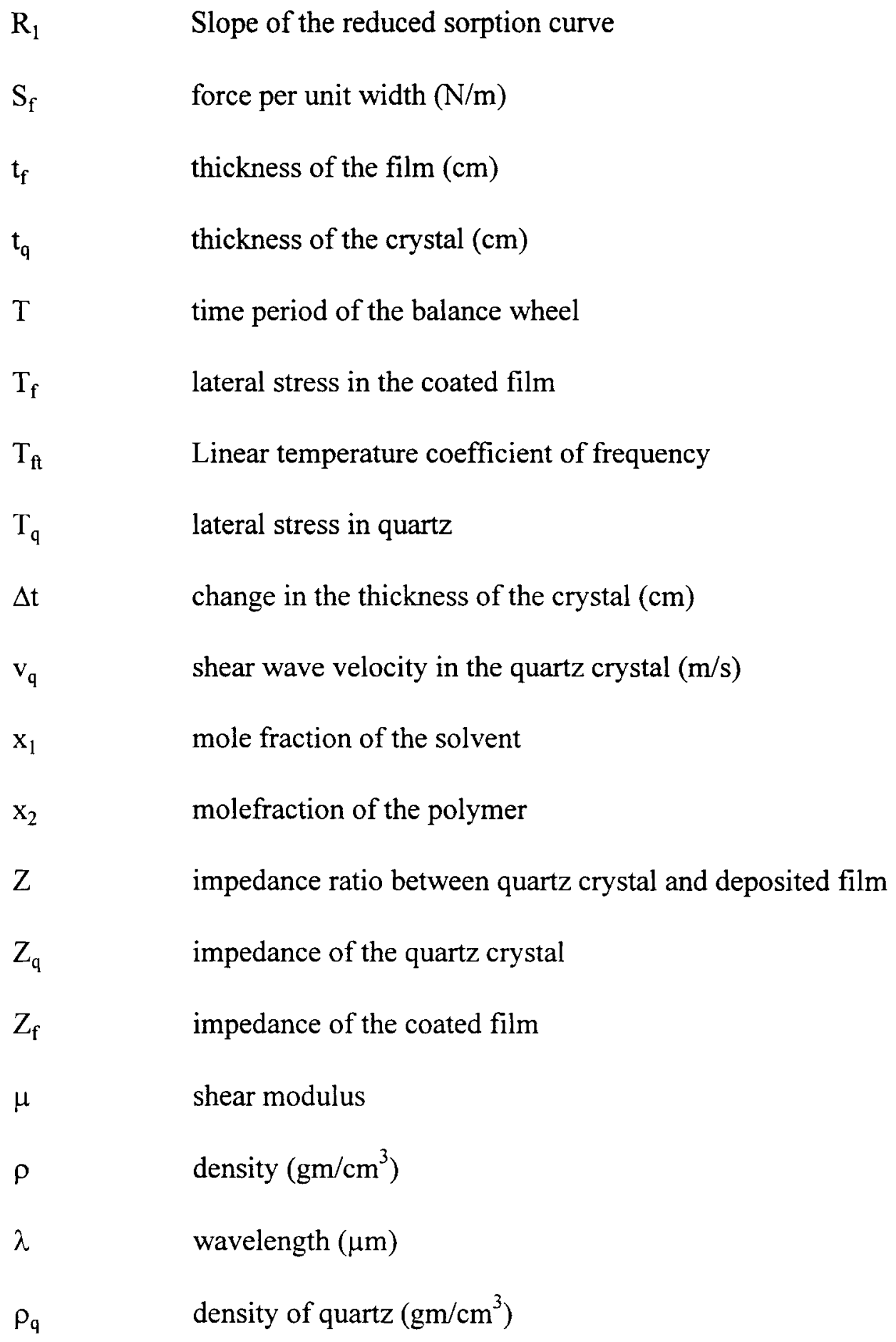




$\begin{array}{ll}\mu_{\mathrm{q}} & \text { shear modulus of quartz } \\ \varepsilon & \text { piezoelectric stress constant } \\ \varepsilon_{\mathrm{o}} & \text { susceptibility of space } \\ \eta_{\mathrm{L}} & \text { viscosity of the liquid } \\ \rho_{\mathrm{L}} & \text { density of the liquid }\left(\mathrm{gm} / \mathrm{cm}^{2}\right) \\ \lambda_{\mathrm{q}} & \text { wavelength of the shear wave in the quartz crystal } \\ \lambda_{\mathrm{m}} & \text { characteristic time of the fluid } \\ \theta_{\mathrm{D}} & \text { characteristic time of the diffusion process } \\ \omega & \text { weight fraction }\end{array}$




\section{CHAPTER I}

\section{INTRODUCTION}

The rate of mass transfer of penetrants in polymers plays an important role in the processing and the end use suitability of polymers. The molecular diffusion of monomers, initiators, plasticizers, and free radicals is particularly important, and the determination of the diffusivity of these low molecular weight compounds in polymers is critical for the accurate modeling of various polymer processes. Knowledge of the diffusion of low molecular weight components is particularly important in polymer coatings, barrier films, controlled drug delivery, and separation processes.

The physical property which quantifies the diffusion mass transfer process is the mutual diffusion coefficient. In systems of low molecular compounds, the diffusion coefficients are usually weak functions of temperature and concentration. If one of the compounds is a polymer, diffusion coefficients are typically strong functions of temperature and concentration and cover a wide range of values $\left(10^{-15}\right.$ to $\left.10^{-5} \mathrm{~cm}^{2} / \mathrm{s}\right)$. In addition, molecular diffusion of small molecules in polymers sometimes does not follow classical laws of diffusion. An understanding of the unique characteristics of polymersolvent diffusion is needed for the detailed analysis of mass transfer problems involving polymer-penetrant systems.

This study exploits the piezoelectric effect in quartz crystals to study diffusion in polymer/penetrant systems. The piezoelectric effect was first discovered by Pierre and Jacques Curie in 1880 (Ward and Buttry, 1990). Ever since, quartz crystals have been 
used extensively as electromechanical transducers and as highly stable oscillators for frequency control. The effect of mass added to the crystal surface on the frequency of a quartz oscillator has been exploited since the early days of radio, when frequency adjustment was made by a pencil mark on the crystal (Lu and Czanderna, 1984). More recently, quartz crystals have been used in various applications in different fields of study (Lu and Czanderna, 1984).

When quartz crystals are used for the measurement of very small masses, the apparatus is called as a quartz crystal microbalance (QCM). QCM's are commonly used for thickness measurements in thin film deposition processes. QCM's are also used for the measurement of stress effects in thin films. QCM's have many applications in surface science and etching studies. QCM's are extensively used in electrochemical studies and in analytical chemistry (Lu and Czanderna, 1984). A detailed description of the application of quartz crystal microbalances is available in Lu and Czanderna (1984).

In this study, a piezoelectric quartz crystal microbalance was used to study the transport of penetrants in polymeric systems. A very thin film of the polymer was coated on the crystal and exposed to a penetrant vapor atmosphere. The mass change of the polymer as the penetrant diffused into the polymer was measured by recording the change in the frequency of the vibrating crystal.

The objective of this study was to gain further understanding of a new sorption technique using a QCM to measure penetrant-polymer mutual diffusivities. The specific objectives of this study were: 
1. To determine if the QCM is a viable and valuable experimental technique for the measurement of thermophysical properties of penetrants in polymers by reproducing the data presented by other researchers for a well studied polymer-solvent system;

2. To demonstrate the advantages and disadvantages of the QCM over other experimental techniques;

3. To demonstrate the applicability of the QCM to industrially relevant systems;

4. To expand the understanding of the working of a quartz crystal in its use as a microbalance.

The ultimate aim of this work is to provide engineers with an accurate, and easy to use experimental technique to measure the thermophysical properties of penetrants in polymers. 


\section{CHAPTER II}

\section{LITERATURE REVIEW}

This thesis represents only the second comprehensive research project involving quartz crystal microbalances at Oklahoma State University. A comprehensive literature review on the piezoelectric effect and its applications in the context of the measurement of penetrant-polymer diffusion has not been previously available. The goal of this chapter is to summarize the previous research relevant to quartz crystal microbalances.

\section{A. THE PIEZOELECTRIC EFFECT}

The piezoelectric effect was discovered more than 100 years ago. In 1880 , Jacques and Pierre Curie discovered that a mechanical stress applied to the surfaces of various crystals induced an electrical potential across the crystal and the magnitude of the electric potential was proportional to the applied stress (Bottom, 1982). The induction of electrical potential in various crystals was referred to as the piezoelectric effect.

After the discovery of the piezoelectric effect, quartz crystals were used in a number of applications. Quartz crystals were used in World War I for generation of sound waves in water for use in submarine detection. Quartz crystals were later used by Langevin for sound wave detection thus employing both the direct and converse piezoelectric effects. Langevin's work was the forerunner of modern SONAR (Bottom, 1982). 
The word piezoelectricity literally means pressure electricity. The name comes from the greek word 'piezein' meaning to press. The production of an electrical polarization by mechanical induction of a strain in a crystal is called the direct piezoelectric effect. The converse effect is production of a mechanical strain in a crystal by a polarizing electric field. The converse piezoelectric effect has been confused with the electrostrictive effect which occurs in solid dielectrics such as glass. The two effects differ in two important aspects. The piezoelectric strain is larger by several orders of magnitude than the constrictive strain, and the piezoelectric strain is proportional to the electric field intensity and changes sign with it. The electrostrictive strain is proportional to the square of the electric field intensity and is therefore independent of its direction. The electrostrictive effect occurs simultaneously with the piezoelectric effect (in quartz) but may be ignored in practice.

The application of polarizing electric fields may cause the crystal to experience a longitudinal or shearing stress and under certain conditions bending, torsion and flexure. The production of mechanical strain from an applied electric field is called the converse piezoelectric field effect (Bottom, 1982). The Curies experimentally verified the converse piezoelectric effect, in which application of a voltage across the piezoelectric crystals induces a mechanical strain (Ward and Buttry, 1990).

The converse piezoelectric effect has been used for the precise control of frequencies in various applications. The application was first used by Cady in 1920 in the construction of very stable oscillator circuits. The application of an alternating electric field across a quartz crystal substrate resulted in an alternating strain field. The 
alternating strain field caused oscillatory motion in the quartz crystal (Ward and Buttry, 1990). Cady's oscillator consisted of a three-stage amplifier with the quartz resonator in a feedback loop. The first broadcast station to be crystal-controlled was WEAF in New York City during 1926. The use of quartz crystals in military equipment was not common until World War II when over 30 million quartz crystals were produced (Bottom, 1982).

Quartz crystals are nearly ideal oscillators because they vibrate with minimal energy dissipation. Their low cost, ruggedness, low defect concentration, easy fabrication and chemical inertness makes them ideal for use in frequency control and filter circuits. The stresses resulting in the crystal depend on the crystal symmetry, angle of cut of the crystal substrate and configuration of the excitation electrodes used to, apply the electric field across the crystal.

\section{B. PIEZOELECTRIC CRYSTALS}

The piezoelectric effect occurs only in certain types of crystals. There are 32 classes of crystals (Example: cubic, trigonal, orthorhombic) of which 20 classes exhibit the piezoelectric effect (Bottom, 1982). The piezoelectric property exists only in ionic crystalline solids that crystallize in structures lacking a center of inversion. The center of inversion is the point about which the crystal is symmetrical. The piezoelectric effect is excluded for all crystals with a center of symmetry (Lu and Czanderna, 1984).

Piezoelectric crystals are used to control and manage the frequencies of the carrier signals used in electric communications. Among the crystals which exhibit the 
piezoelectric effect, quartz alone provides the necessary combination of mechanical, electrical, chemical and thermal properties required for making piezoelectric elements for the electrical communication field (Bottom, 1982). The quartz crystal unit provides a coupling between the mechanical resonance frequency of the crystal and the electric circuit, and, therefore, can be used as an electromechanical transducer. The coupling between the mechanical properties of the quartz and the electric circuit occurs through the piezoelectric effect.

The piezoelectric effect occurs because of the generation of dipoles in crystals upon the application of an electric field. A piezoelectric crystal is a precisely cut slab from a crystal of quartz. The crystals are cut at a specific angle and modified to suppress the unwanted modes. There are several types of crystal cuts such as AT-cut crystals, BTcut crystals and SC-cut crystals. The crystals differ in the angle of cut. The application of an electric potential to a quartz crystal produces internal mechanical stress. In quartz crystals, an applied pressure deforms the crystal lattice and causes separation of the centers of positive and negative charge, generating a dipole moment in each crystal cell. When a quartz crystal is connected to a periodic voltage source, the crystal vibrates at the frequency of the exciting voltage. The vibration causes the contraction or elongation of the crystal parallel to the direction of the applied electric field. If the frequency of the voltage source is close to one of the mechanical resonant frequencies of the quartz crystal, resonance occurs and the amplitude of the vibration reaches a maximum.

A piezoelectric quartz crystal has many modes of resonance. For example, a rectangular bar can have longitudinal (extensional), lateral (flexural and shear), and 
torsional (twist) motion in each of the three axes. The crystal can also vibrate in overtones of each of the fundamental modes. The selection of a particular mode of vibration depends upon the specific crystallographic orientation of the cut and the shape of the crystal. The mode of vibration which is most sensitive to the addition or removal of mass for a quartz crystal is called the high frequency thickness-shear mode. For the crystal to oscillate in the thickness-shear mode, the crystal must be cut to give a specific orientation with respect to the crystal axes.

Crystalline quartz is composed of two elements, silicon and oxygen and the chemical formula is $\mathrm{SiO}_{2}$. The amorphous form of $\mathrm{SiO}_{2}$ is a major constituent of many rocks and most sands. The crystalline form of $\mathrm{SiO}_{2}$ or quartz is relatively abundant in nature, but not in the highly perfect form required for the manufacture of quartz crystal units. Most of the high quality crystals of natural quartz are from Brazil. Natural quartz crystals are grown from water solutions containing dissolved $\mathrm{SiO}_{2}$. Silicon dioxide is slightly soluble in water at ordinary temperatures. However the solubility increases appreciably in alkaline solutions. Crystals of cultured quartz are grown by dissolving $\mathrm{SiO}_{2}$ in an alkaline solution of water at the temperature near the critical temperature of quartz. Autoclaves which can withstand pressures exceeding 1000 atm are required for the preparation of cultured quartz. Seed crystals are placed in the cooler portions of the autoclave, and feedstock in the form of chunks of $\mathrm{SiO}_{2}$ in the warmer portion. The solution moves by convection from the hotter region to the cooler region dissolving the nutrient and depositing on the seed crystals. The quality of quartz crystals depends on the conditions of the growth. The differences between the samples of quartz are due to the 
crystalline imperfections. The crystalline form of $\mathrm{SiO}_{2}$ below $573{ }^{\circ} \mathrm{C}$ is called alpha quartz (Bottom, 1982).

Piezoelectric quartz crystals have three important properties:

1. The piezoelectric effect provides a coupling between the electrical circuit and the mechanical properties of a crystal.

2. The internal dissipation is low.

3. The density and elastic constants of the crystals are uniform, so that a crystal cut at a given orientation will always have the same frequency behavior.

4. Crystals cut at a specific orientation have advantageous properties.

5. The crystals have a small frequency change with temperature and will be free from secondary modes of motion (Heising, 1947).

Resonance can occur in quartz crystals at different frequencies. Resonance in crystal resonators occurs at all the frequencies where mechanical displacements of the elastic medium form standing wave patterns. In resonator terminology, these patterns are called resonance modes of motion. The simplest mode patterns (i.e., the standing wave patterns of the fundamental mode) occur at the fundamental frequencies of the low frequency resonators. 
The quartz crystal in an oscillator circuit is analogous to the balance wheel and hair spring in a watch. In both cases the function of a quartz crystal is to provide isochronous vibrations. The time period of the balance wheel in a watch is

$$
\mathrm{T}=2 \pi \sqrt{\mathrm{l} / \mathrm{h}} \text {, }
$$

where $\mathrm{I}$ is the moment of inertia of the balance wheel and $\mathrm{h}$ is the stiffness coefficient of the hair spring. The above equation is only true for a frictionless vibrating system. Since no vibrating system is frictionless, energy must be supplied to sustain oscillations. Even in the case of crystal vibrations, energy must be supplied to sustain the oscillations.

The energy is supplied to the vibrating crystal through the piezoelectric effect. In crystal oscillations, the energy losses are extremely small. The properties of quartz which makes quartz the most satisfactory material for frequency stabilization are hardness, low internal dissipation, durability, uniformity, freedom from flaws and temperature independent resonant frequencies. There are other crystals which satisfy the above requirements, but none except quartz satisfy all of them.

\section{AT-CUT QUARTZ CRYSTALS}

AT cut quartz crystals are the most widely used quartz crystals. AT cut quartz crystals are obtained by cutting wafers of quartz at approximately $35^{\circ}$ from the $z$-axis as shown in Figure 1. The length direction before the rotation is the z-axis. Application of 


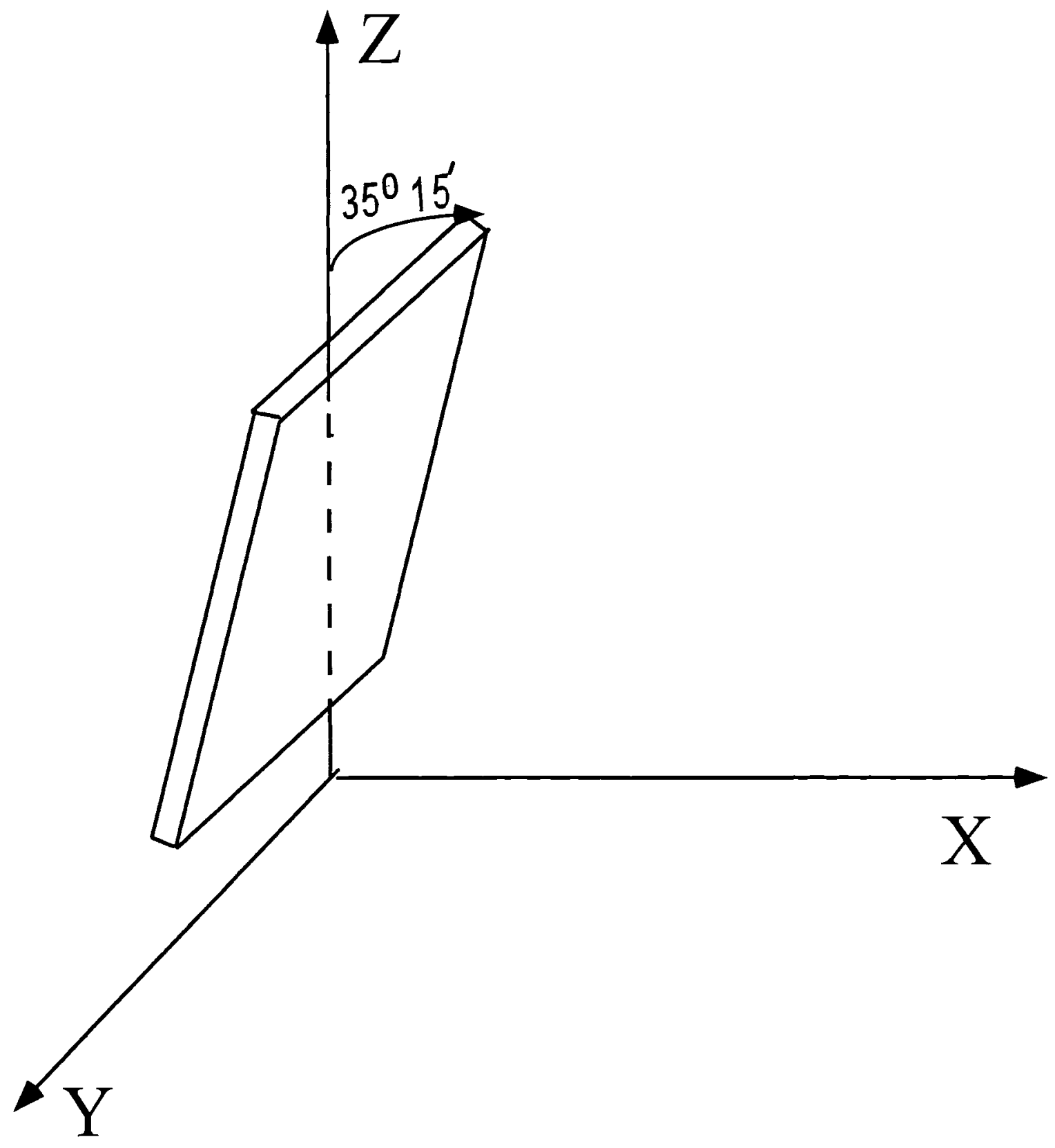

Figure 1.

Angle of Cut and Orientation of an AT-cut quartz Crystal 
electric field across the thickness of an AT cut quartz crystal results in a shear vibration in the direction parallel to the field and propagation of a shear wave through the crystal in the thickness direction.

For an AT-cut quartz crystal, the thickness-shear mode is the fundamental mode and the mode with the simplest mode pattern. The resonance frequencies of the elementary modes are controlled by the major dimensions of the resonator, but at higher frequencies where the wave length of the elastic waves becomes comparable to the small dimensions of the body, two additional types of elementary motion exist. In the case of AT-cut quartz crystals, they are the anharmonic thickness-shear mode and thickness-twist overtones of the fundamental thickness shear mode. All of these modes are, in general grouped together closely in frequency (Hafner, 1974). All the overtone modes have more complex standing wave patterns than the fundamental mode.

For the AT-cut quartz crystal operating in the thickness shear mode, the vibration of the crystal is confined to the region between the two electrodes (i.e., the thickness of the crystal). In case of fundamental shear mode of the AT-cut quartz crystal, the resonant frequency is

$$
f_{o}=(\mu / \rho)^{1 / 2 / 2 t_{q}}
$$

where $\mu$ and $\rho$ are the shear modulus $\left(2.947 \times 10^{11} \mathrm{dyne} / \mathrm{cm}^{2}\right)$ and density $\left(2.648 \mathrm{~g} / \mathrm{cm}^{3}\right)$ of quartz, respectively and $t_{\mathrm{q}}$ is the thickness of the crystal. Increasing the thickness of the crystal increases the wavelength $\left(\lambda=2 t_{q}\right)$ and decreases the fundamental frequency of vibration. 
AT-cut quartz crystals are widely used for the determination of small mass changes on the surface of the crystals. A description and experimental proof of the massfrequency relation for foreign layers deposited on the crystals oscillating in the thickness shear mode was provided by Sauerbrey $(1957,1959)$. The derivation of the massfrequency relationship by Sauerbrey was based on the assumption that a deposited foreign material existed entirely at the antinode (i.e., the point of maximum amplitude) of the standing wave, i.e., treating the foreign deposit as an extension of the quartz crystal. The frequency change would be calculated as though it were the result of an increase in the thickness of the quartz crystal. Sauerbrey (1957 and 1959) assumed a uniform distribution of mass on the entire electrode portion of an AT cut quartz crystal. According to Sauerbrey (1957 and 1959),

$$
\frac{\Delta f}{f_{o}}=-\frac{\Delta t}{t_{q}}
$$

and the appropriate substitution of Equation (2) in Equation (3) gives

$$
\Delta \mathrm{f}=\frac{-2 \mathrm{f}_{0} \Delta \mathrm{m}}{\mathrm{A} \sqrt{\rho_{\mathrm{q}} \mu_{q}}},
$$

where $\Delta \mathrm{f}$ is the measured frequency shift, $\Delta \mathrm{t}$ is the change in the thickness of the crystal due to coating, $f_{0}$ is the fundamental frequency of the quartz crystal prior to a mass change, $\Delta \mathrm{m}$ is the mass change, $\mathrm{A}$ is the piezoelectrically active area, $\rho_{\mathrm{q}}$ is the density of quartz, and $\mu_{\mathrm{q}}$ is the shear modulus. Consequently, a change in the mass per unit area results in a corresponding change in the frequency. Equation (4) is valid only for small films with an acoustic impedance identical to that of quartz (Ward and Buttry, 1990). 
The electrical analogue of the pressure difference across an acoustic element is the voltage across the corresponding element of the electrical circuit. The acoustic analogue of the current in a circuit is the volume velocity of the fluid in the corresponding acoustic element. Therefore, the acoustic impedance of a fluid medium acting on or through a surface is the complex quotient of acoustic pressure divided by the volume velocity at the surface. The impedance of a portion of the acoustic system is defined as the complex ratio of the pressure difference effective in driving that portion to a volume velocity (Kinsler and Frey, 1962).

Circular AT-cut quartz plates are usually contoured to give one or both of the major surfaces a spherical shape. Electrodes smaller than the quartz plate are deposited on the major surfaces, concentric with the plate. Under these conditions the vibrating region is confined to a circular region which may be smaller, equal to, or larger than the area of electrodes. The displacement of the surface of the quartz plate can be observed by permitting some fine dust to fall on the surface of the quartz while it is excited. The dust is driven from the regions of maximum motion to collect along the nodes (Bottom, 1982).

The vibration amplitude of quartz-crystals oscillating in thickness-shear mode are typically less than $50 \mathrm{~nm}$. The knowledge of the amplitude distribution on the crystal surface is important for the analysis of the mass load sensitivity of the quartz crystal when used as a thin-film thickness monitor (Wimmer et al., 1984). The sensitivity of the quartz crystal to mass changes decreases from the center of the electrode to the electrode edge (Ward and Buttry, 1990). To achieve high accuracy in calculating the film thickness by evaluating the frequency shift of the resonator, the sensitivity of the frequency change 
due to the mass load is considered to be dependent on the distance from the center. So there is a different integral sensitivity depending on the diameter of the deposited film. The difference in integral sensitivity is caused by the locally varying vibration amplitude. The vibration amplitudes of quartz crystal vibrations can be observed by X-ray topography, neutron diffraction and scanning electron microscope (Wimmer et al., 1984).

\section{EQUIVALENT CIRCUIT OF THE QUARTZ RESONATOR}

A quartz resonator can be represented by an equivalent electrical circuit. The crystal resonator is really an electromechanical transducer (i.e., a device which can interchange electrical and mechanical energy). Several years before the discovery of the piezoelectric resonator, Dye (1926) and Butterworth (1915) showed that any mechanically vibrating system driven by means of an electromagnetic field and electrostatic field can be represented by the circuits given in Figure 2. The relationship between the parameters of the electrical network and the physical properties of the piezoid were developed by Van Dyke (1928), Butterworth (1915) and others. The equivalent circuit of a piezoelectric transducer is shown in Figure 2b. The electrical parameters in terms of the physical properties of the quartz are given by

$$
\begin{aligned}
& \mathrm{C}_{\mathrm{o}}=\mathrm{k} \varepsilon_{\mathrm{o}} \frac{\mathrm{A}}{\mathrm{e}}, \\
& \mathrm{R}=\frac{\mathrm{e}^{3} \mathrm{r}}{8 \mathrm{~A} \varepsilon^{2}}, \\
& \mathrm{~L}=\frac{\mathrm{e}^{3} \rho}{8 \mathrm{~A} \varepsilon^{2}}, \text { and }
\end{aligned}
$$




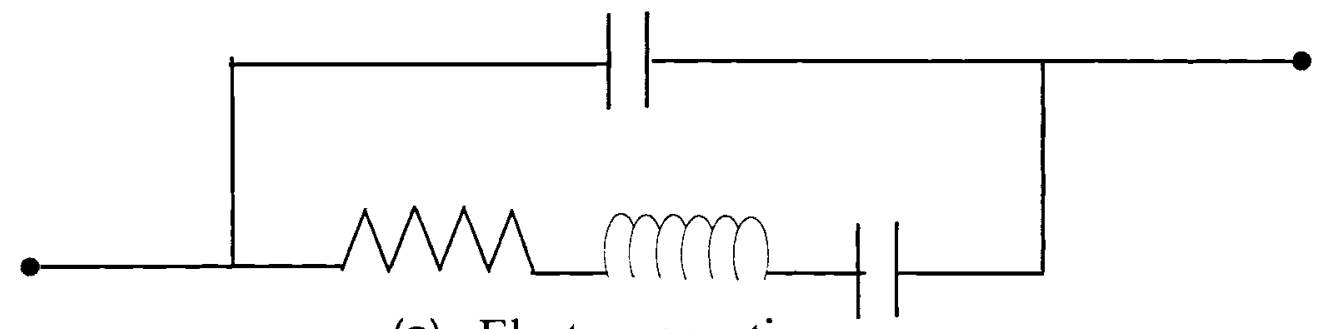

(a) Electromagnetic

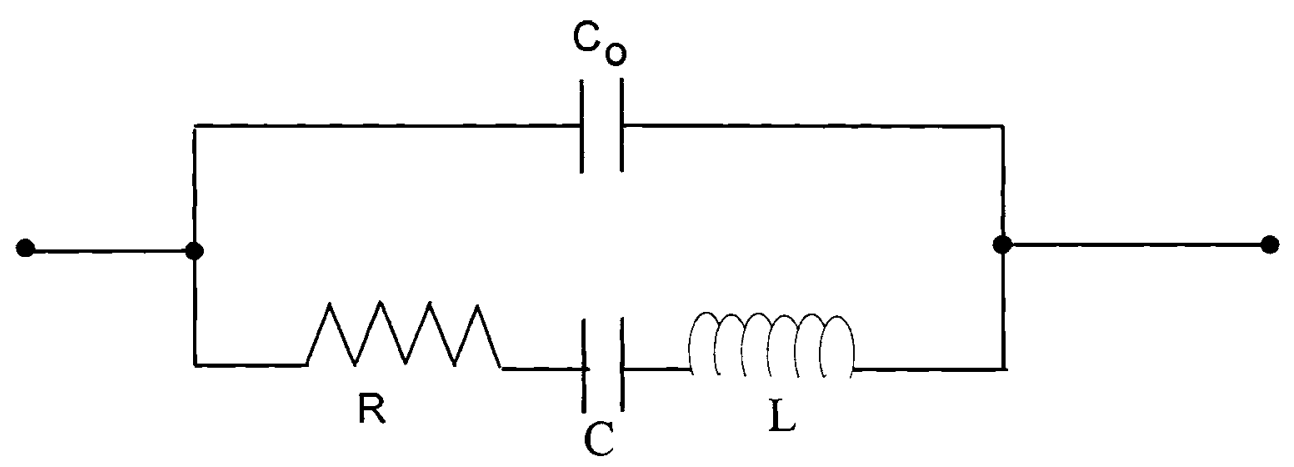

(b) Electromechanical

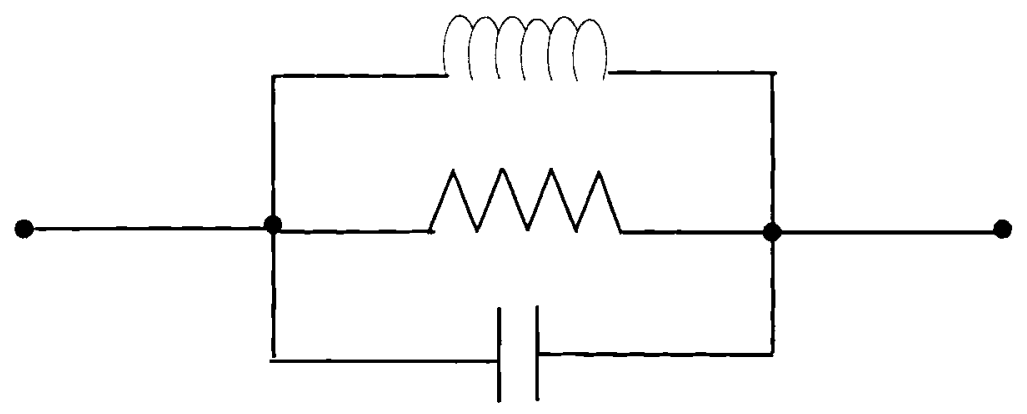

(c) Electrostatic

Figure 2

Equivalent Circuits of Electromagnetic, Electromechanical and Electrostatic Transducers 


$$
\mathrm{C}=\frac{8 \mathrm{~A} \varepsilon^{2}}{\pi^{2} \mathrm{ec}}
$$

where

$$
\begin{aligned}
& \mathrm{A}=\text { area of the plate, } \\
& \mathrm{e}=\text { thickness of the plate, } \\
& \mathrm{c}=\text { elastic constant } \\
& \varepsilon=\text { piezoelectric stress constant, } \\
& \rho=\text { density of quartz }(2.65 \mathrm{gm} / \mathrm{cc}), \\
& \mathrm{r}=\text { damping coefficient }, \\
& \varepsilon_{0}=\text { susceptibility of space, and } \\
& \mathrm{k}=\text { dielectric constant. }
\end{aligned}
$$

It is impossible to simulate the behavior of the quartz crystal unit with electrical components other than quartz crystals in a circuit. The value of $\mathrm{C}$ in a typical 5-MHZ AT-cut quartz crystal is of the order of $0.01 \mathrm{pF}$, and a capacitor of this size cannot be easily built. The value of $L$ is of the order of $0.1 \mathrm{H}$ and $\mathrm{R}$ is of the order of $10 \Omega$. A coil having an inductance of $0.1 \mathrm{H}$ requires an excessive number of turns of wire on a ferromagnetic core. The construction of such a coil with a resistance of only $10 \Omega$ in a volume of less than $0.5 \mathrm{~cm}^{3}$ is impossible (Bottom, 1982).

In the equivalent electrical circuit, the capacitance $\mathrm{C}$ represents the mechanical elasticity of the vibrating body; the inductance $\mathrm{L}$ is a measure of the vibrating mass and resistance $\mathrm{R}$ corresponds to the total loss of mechanical energy due to internal friction and other energy dissipations (example: viscous dissipations). The shunt capacitance $C_{0}$ is the capacitance due to the electrodes on the crystal and other supporting structures. 
For resonance to occur, the complex impedance of the quartz crystal resonator must be resistive only at the resonance frequency. The resonator impedance becomes resistive at two frequencies:

$$
\begin{aligned}
& \mathrm{f}_{\mathrm{s}}=\frac{1}{2 \pi} \sqrt{\frac{1}{\mathrm{LC}}} \text {, and } \\
& \mathrm{f}_{\mathrm{p}}=\frac{1}{2 \pi}\left[\left(\frac{1}{\mathrm{LC}}\right)+\left(\frac{1}{\mathrm{LC}}\right)+\left(\frac{\mathrm{R}^{2}}{\mathrm{~L}^{2}}\right)\right]^{1 / 2}
\end{aligned}
$$

The two resonant frequencies are called series and parallel resonances, respectively. Quartz crystals used in microbalances operate in series resonance mode. From the above equations it can be seen that the series resonant frequency is not affected by the shunt capacitance $\mathrm{C}_{0}$. The mass sensitivity can be considered as a perturbation in the inductance $\mathrm{L}$. An increase or decrease in the mass changes the value of $\mathrm{L}$. Accordingly the resonant frequency changes depending on the mass change, and this provides a phenomenological explanation of the operation of the quartz crystal as a microbalance. Exact values of $\mathrm{L}$ and $\mathrm{C}$ cannot be derived from the physical properties of the quartz crystal (Lu and Czanderna, 1984).

\section{THE TEMPERATURE COEFFICIENT OF FREQUENCY FOR QUARTZ}

\section{CRYSTALS}

The frequency of any resonator depends on its thickness, density, and the elastic constant and each of these changes with the temperature. Therefore, the frequency of any resonator is usually temperature dependent; however, the frequency of an AT cut quartz crystal is not a function of temperature through a convenient balancing of the properties 
of the crystal. For an infinite plate vibrating in thickness mode, the resonant frequency is given by

$$
f_{n}=\frac{n}{2 e} \sqrt{\frac{c_{i j}}{\rho}} \quad n=1,3,5
$$

where

$$
\begin{aligned}
& \mathrm{e}=\text { thickness of the plate, } \\
& \rho=\text { density of quartz, and } \\
& \mathrm{c}_{\mathrm{ij}}=\text { ratio of the stress to the strain associated with the } \\
& \text { elastic wave which is being propagated in the quartz. }
\end{aligned}
$$

Assuming linear temperature dependence of the above variables, the linear temperature coefficient of the frequency, $\mathrm{T}_{\mathrm{ft}}$ is given by

$$
T_{\mathrm{ft}}=\frac{1}{\mathrm{f}} \frac{\mathrm{df}}{\mathrm{dT}}=-\frac{1}{\mathrm{e}} \frac{\mathrm{de}}{\mathrm{dT}}-\frac{1}{2} \frac{1}{\rho} \frac{\mathrm{d} \rho}{\mathrm{dT}}
$$

Since quartz expands on heating, $\frac{\mathrm{d} \rho}{\mathrm{dT}}$ is negative. The change in thickness with temperature is $\frac{d e}{d T}$ and is a positive quantity. $T_{\mathrm{ft}}$ can be made zero by balancing out the effects of temperature on density and elastic modulus so that the temperature dependence of frequency can be minimized. This balancing out of the effects is possible in AT-cut quartz crystals. For most other crystals it is impossible to find any orientation with zero temperature coefficient (Bottom, 1982). 


\section{OSCILLATION OF QUARTZ CRYSTALS IN LIQUIDS}

Quartz crystals oscillate even when immersed in liquids if the viscosity of the liquid is not large. The oscillation of quartz crystals in liquids is contrary to the belief that the viscous losses to the liquid would suppress the oscillation of the crystals (Reed et al., 1990). When the quartz crystals oscillate in a liquid medium, the frequency is found to be dependent on the viscosity and density of the liquid in contact with the crystal, according to

$$
\Delta f=-f_{0}^{/ 2} \sqrt{\frac{\eta_{L} \rho_{L}}{\pi \mu_{q} \rho_{q}}},
$$

where $\eta_{\mathrm{L}}$ and $\rho_{\mathrm{L}}$ are the viscosity and the density of the liquid in contact, respectively. The parameters $\rho_{q}$ and $\mu_{q}$ are the density and elastic modulus of the quartz and $f_{o}$ is the oscillation frequency of the bare crystal. This relation has been experimentally tested by Lin et al. (1993) with a $30 \mathrm{MHz}$ quartz crystal. In liquids, the amplitude of oscillation of the quartz crystal decreases because the liquid viscosity and the liquid density increase the shear stress on the crystal. The amplitude of oscillation is proportional to the net driving force on the crystal. The net driving force is equal to the difference between the piezoelectric force and the shear stress because the shear stress reduces the net force on the crystal (Martin and Hager, 1989). Any mass accumulated on a crystal oscillates synchronously with the crystal surface and reduces the resonant frequency of the crystal. When the crystal surface is in contact with a liquid, a thin layer of liquid adjacent to the oscillating crystal is entrained by the crystal surface, further reducing the frequency of the crystal (Klavetter et al., 1993). The oscillating crystal sets up an oscillating liquid 
boundary layer adjacent to the face in contact with the liquid (Bruckenstein and Shay, 1985). So mass accumulation on the surface and liquid contact both lead to a decrease in the resonant frequency of the crystal. The sensitivity of the resonant frequency of the crystal to liquid properties can be used in monitoring the liquid density and viscosity (Klavetter et al., 1993).

Yao and Zhou (1988) studied the effect of the properties of the liquids on the oscillation of quartz crystals. They found that, for a crystal immersed in a liquid there exists a critical temperature below which the crystal ceases to oscillate. The temperature at which the crystal ceases to oscillate differs for different crystals even with the same rated frequency. Yao and Zhou (1988) concluded that the crystals oscillate with greater difficulty in concentrated solutions and in liquids with high molecular weight. They also compared the effects of density and viscosity of the liquids on the frequency behavior the crystals and concluded that the influence of density is more significant than the influence of viscosity on the frequency of the crystals (Yao and Zhou, 1988). Nomura and Okuhara (1982) performed experiments with quartz crystals in various liquids and suggested an empirical equation that the relates the frequency shifts of the crystals to the density and viscosity of the liquid. They also indicated that the crystals did not oscillate in some of the liquids tested.

\section{STRESS EFFECTS IN QUARTZ CRYSTALS}

The frequency of vibration of a quartz crystal depends on the dimensions of the quartz crystal, the density of quartz and the elastic stiffness of quartz. All three quantities 
vary when there is static stress or initial stress present in the quartz crystal. The resonant frequency of the crystal changes with static stress. Two main sources of static stress are: mechanical sources such as the electrodes and thermal shocks arising from ambient temperature changes. Quartz crystal microbalances can also be used for measuring the stress effects in thin films that result from film deposition, sputtering, ion implantation, or chemical reactions in thin films. The magnitude of stress effects in a quartz crystal may be or may not be important in a given situation.

The stresses arising from materials on the surface of the quartz crystal are shown in the Figure 3. In normal usage of the quartz crystal as a microbalance, changes in the resonant frequency are observed due to mass changes on the surface of the crystal. When stress builds in the thin film on the surface of the crystal, there is a net force per unit width acting across the thin film and quartz interface that stress biases the quartz. The frequency changes caused by this stress bias are referred to as the "stress effects".

The force per unit width acting on the quartz crystal is related to the lateral stress in the deposited thin film. The lateral stress may be the intrinsic stress arising in the thin films due to the nucleation process (i.e., a process which occurs before the growth of the films on the crystal surface). The lateral stress can increase during sputtering or ion implantation. When the bombarding particles strike the surface of the already deposited film during the film growth (i.e. by sputtering or ion implantation), the bombarded layer can have lattice structural damages. When the bombarded layer tries to expand or contract from the structural damages, the lateral stress can increase. Sputtering and ion implantation are two different types of procedures by which metallic films are grown. A 


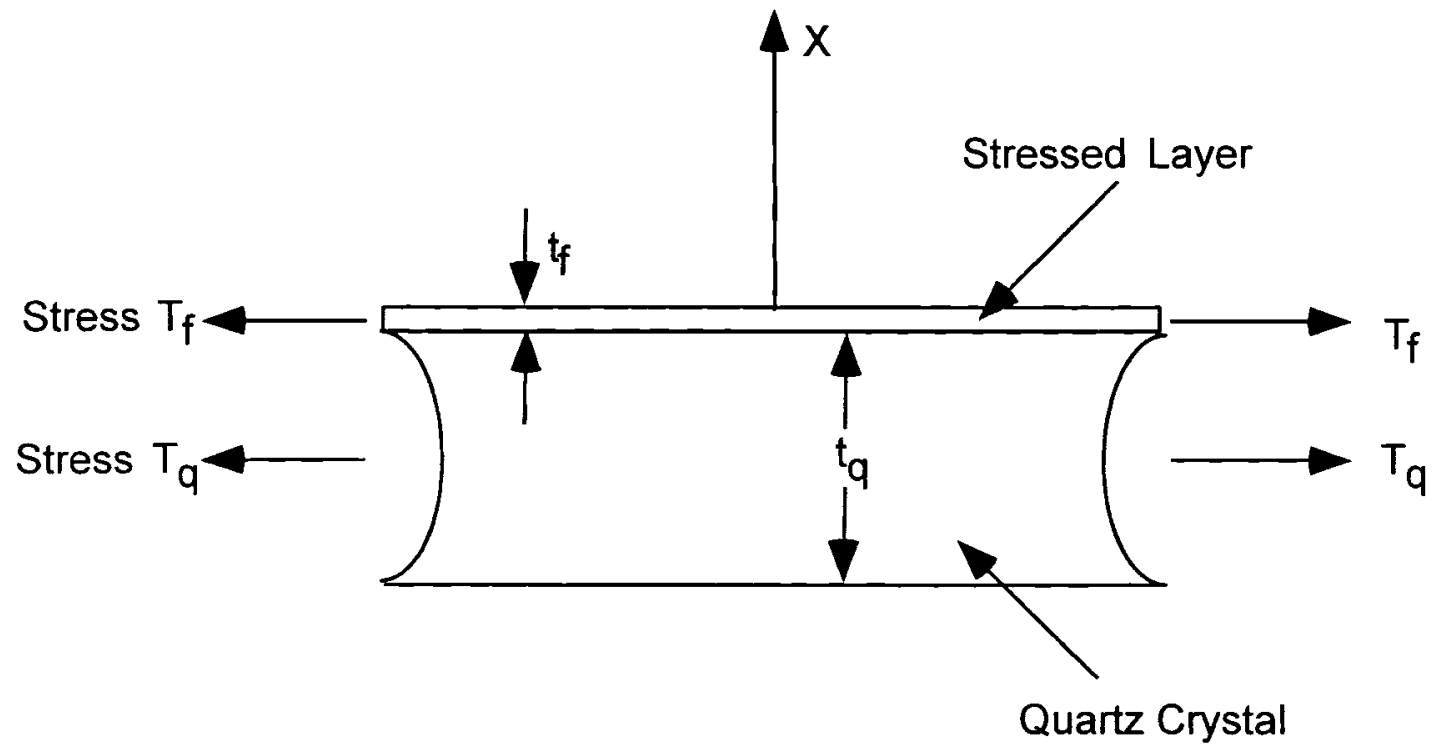

Figure 3.

Stresses in the Quartz Crystal after Coating 
lateral stress can also arise from the free volume changes associated with chemical reactions in the coated film. The force per unit width in $\mathrm{N} / \mathrm{m}$ is given by $\mathrm{S}_{f}$, the integral through the film thickness,

$$
S_{f}=\int_{0}^{t_{f}} T_{f} d x
$$

where $t_{f}$ is the thickness of the deposited film that has the lateral stress, $x$ is the coordinate normal to the quartz resonator surface, and $T_{f}$ is the lateral stress in the deposited film. According to Newton's law, there must be an equal and opposite force per unit width acting at the interface from the quartz resonator side. This results in a lateral stress $T_{q}$ in the quartz. Assuming a uniform lateral stress, the lateral stress $T_{q}$ in the quartz resonator is given by

$$
T_{q}=-\frac{S_{f}}{t_{q}}
$$

where $t_{q}$ is the thickness of the quartz crystal. This is the stress bias that changes the resonant frequency of the quartz crystal. According to the sign convention, a positive value of $T_{q}$ means tension in the quartz resonator. The presence of negative sign in Equation (15) is because the stress in the quartz resonator would be opposite to the stress in the thin film.

According to EerNisse (1972), the fractional frequency change $\mathrm{df}_{\mathrm{S}} / \mathrm{f}$, due to the lateral stress in the quartz resonator is given by

$$
\frac{\mathrm{df}_{\mathrm{s}}}{\mathrm{f}}=\mathrm{KT}_{\mathrm{q}},
$$


where $\mathrm{K}$ is a constant for a given crystallographic orientation of the quartz crystal. The two most commonly used crystals are the AT-cut and the BT-cut quartz crystals. The parameter $\mathrm{K}$ has the values of $2.75 \times 10^{-11}$ and $2.65 \times 10^{-11} \mathrm{~m}^{2} \mathrm{~N}$ for the AT and BT cut crystals, respectively. The combination of Equations (15) and (16) can be written as

$$
\frac{\mathrm{df}_{\mathrm{s}}}{\mathrm{f}}=-\mathrm{K} \frac{\mathrm{S}_{\mathrm{f}}}{\mathrm{t}_{\mathrm{q}}}
$$

The value of $\mathrm{K}$ for an SC-cut quartz crystal is zero. The SC cut orientation is the only crystallographic orientation in quartz with both stress compensation and temperature compensation available in its fundamental resonance mode. So, mass changes on the surface of the quartz resonator can be accurately measured free of errors arising from stress-related effects, with the usage of an SC-cut quartz crystal.

\section{QUARTZ CRYSTAL MICROBALANCES}

One of the major uses of quartz crystals is in microbalances. In a quartz crystal microbalance, mass change is detected by detecting the change in resonant frequency. The use of piezoelectric quartz crystals as mass measuring devices was first explored by Sauerbrey $(1957,1959)$. The operating principle can be described by an idealized physical model shown in Figure 4. Part (a) of Figure 4 shows the oscillation of a bare quartz crystal oscillating in its fundamental thickness-shear mode. The dotted lines in

part (a) and part (b) of Figure 4 represent the shear waves in the quartz crystal. For the quartz crystal to oscillate in thickness shear mode, the thickness of the crystal should be 


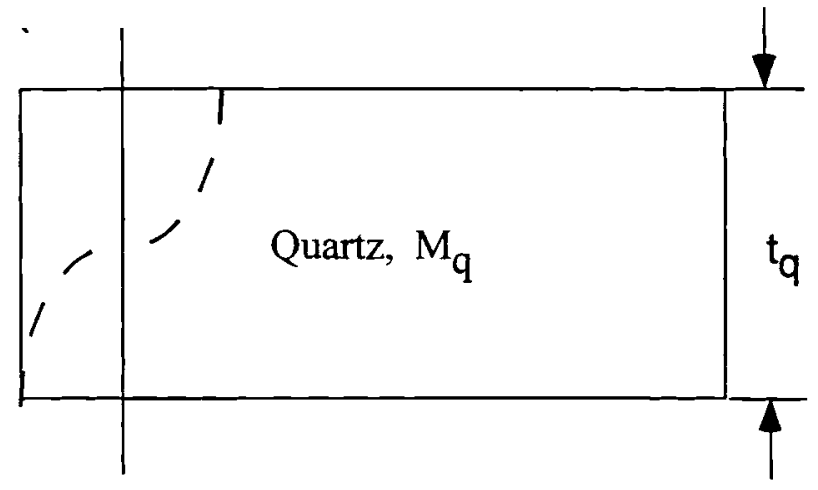

(a)
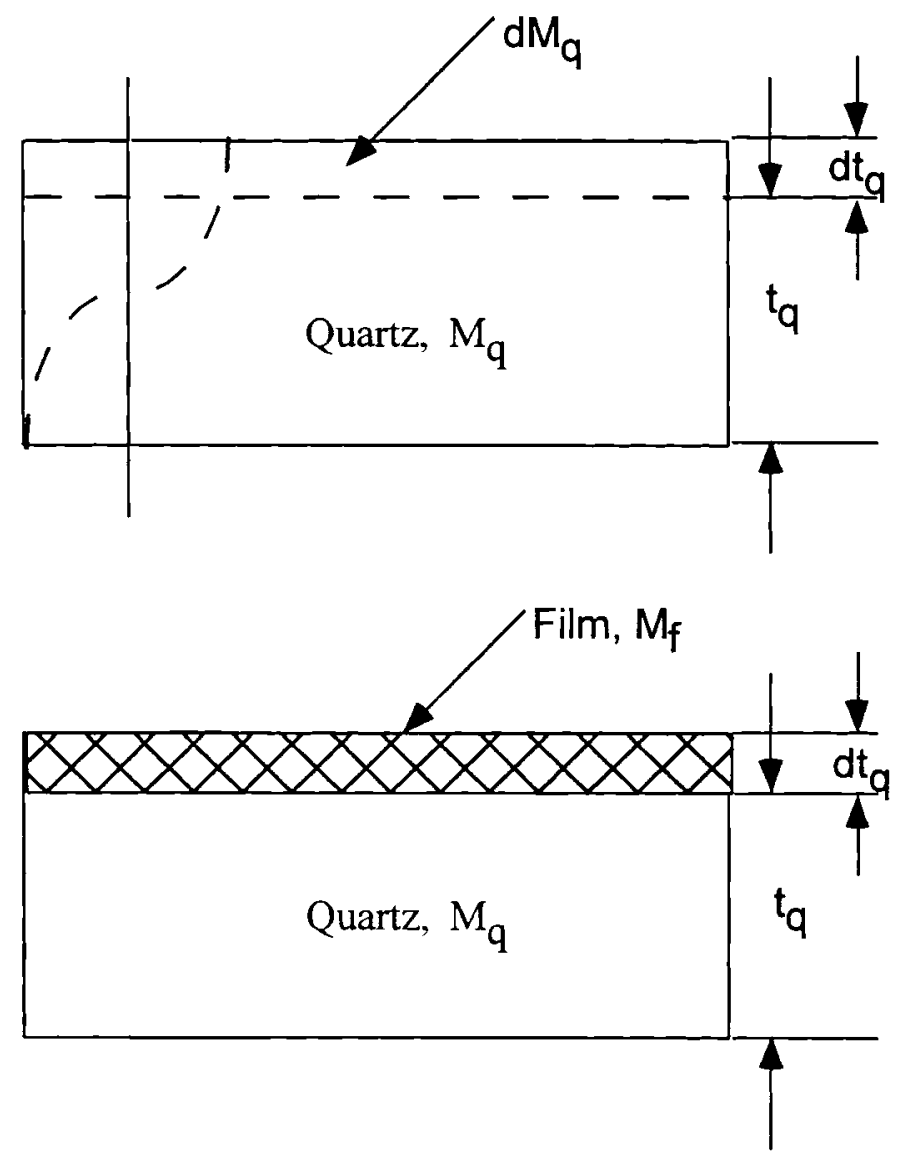

(c)

Figure 4 .

Physical Model of the Coated Quartz Crystal 
exactly half the wavelength of the shear-mode elastic waves in the thickness direction. This condition can be mathematically represented as,.

$$
\mathrm{t}_{\mathrm{q}}=\frac{\lambda_{\mathrm{q}}}{2}
$$

where $t_{q}$ is the thickness of the crystal and $\lambda_{q}$ is the wavelength of the shear-mode elastic wave in the thickness direction. The effects of the electrodes are ignored in this model. The above equation can also be written as

$$
\mathrm{ft}_{\mathrm{q}}=\mathrm{v}_{\mathrm{q}} / 2 \text {, since } \lambda_{\mathrm{q}} \mathrm{f}_{\mathrm{q}}=\mathrm{v}_{\mathrm{q}} \text {. }
$$

$f_{q}$ is the frequency of the bare quartz crystal and $v_{q}$ is the shear wave velocity in the crystal. The frequency shift of the quartz crystal $\mathrm{df}_{\mathrm{q}}$ caused by an infinitesimal change in crystal thickness $\mathrm{dt}_{\mathrm{q}}$ is found to be

$$
\frac{\mathrm{df}_{\mathrm{q}}}{\mathrm{f}_{q}}=-\frac{\mathrm{dt}_{\mathrm{q}}}{\mathrm{t}_{q}},
$$

If the above equation is expressed in terms of mass of the quartz crystal $\mathrm{M}_{\mathrm{q}}$, and the mass change $\mathrm{dM}_{\mathrm{q}}$, it can be written as,

$$
\frac{d f_{q}}{f_{q}}=-\frac{d M_{q}}{M_{q}} .
$$

The oscillation of the crystal under these conditions is shown in part (b) of Figure 4.

Sauerbrey assumed that

1. The material coated on the crystal is perfectly bound to the electrode. 
2. The material coated on the crystal is elastic and does not undergo viscoelastic deformations during oscillation.

3. The thickness of the coated material is uniform over the crystal surface.

4. The acoustic impedance of the crystal is identical to that of quartz.

All the above conditions can be satisfied when the material thickness is small compared to the quartz plate thickness (Nö̈l and Topart, 1994). Based on the above assumptions, for small changes in mass, the addition of foreign mass can be treated as an equivalent mass change of the quartz crystal itself, and the above equation can be written as

$$
\frac{d f_{q}}{f_{q}}=-\frac{d M}{M_{q}},
$$

where $\mathrm{dM}$ is an infinitesimal amount of foreign mass distributed uniformly over the surface of the crystal. Equation (22) can also be written as

$$
\frac{\left(f_{c}-f_{q}\right)}{f_{q}}=-\frac{M_{f}}{M_{q}},
$$

where $f_{c}$ is the resonant frequency of the quartz crystal with the deposited material and $M_{f}$ is the mass of the foreign substance added to the crystal, as is shown in part (c) of Figure 4. If the above equation is written in terms of areal densities (mass per unit area)

$$
\frac{\left(f_{c}-f_{q}\right)}{f_{q}}=-\frac{m_{f}}{m_{q}}
$$


where

$$
\begin{aligned}
& \mathrm{m}_{\mathrm{f}}=\mathrm{t}_{\mathrm{f}} \rho_{\mathrm{f}}, \\
& \mathrm{m}_{\mathrm{q}}=\mathrm{t}_{\mathrm{q}} \rho_{\mathrm{q}}, \\
& \mathrm{m}_{\mathrm{q}}=\text { areal density of quartz crystal, } \\
& \mathrm{m}_{\mathrm{f}}=\text { areal density of the film, } \\
& \rho_{\mathrm{f}}=\text { density of the film, } \\
& \rho_{\mathrm{q}}=\text { density of quartz, } \\
& \mathrm{t}_{\mathrm{f}}=\text { thickness of the film, and } \\
& \mathrm{t}_{\mathrm{q}}=\text { thickness of the quartz crystal. }
\end{aligned}
$$

From the Equations (18)-(26)

$$
m_{f}=\frac{-\left(f_{c}-f_{q}\right) \rho_{q} v_{q}}{2 f_{q}^{2}},
$$

or

$$
\Delta \mathrm{f}=-\mathrm{C}_{\mathrm{f}} \mathrm{m}_{\mathrm{f}}
$$

where

$$
C_{f}=\frac{2 f_{q}^{2}}{\rho_{q} v_{q}}
$$

$\mathrm{C}_{\mathrm{f}}$ is called the mass sensitivity of the crystal and $\Delta \mathrm{f}$ is the frequency change to addition of a foreign substance.

The mass sensitivity of the crystal can be calculated from the resonant frequency and the intrinsic properties of the crystal. According to Sauerbrey (1957 and 1959), the mass sensitivity is independent of the physical properties of the deposited material. This was experimentally verified by several investigators using crystals with varying frequencies and with various deposition materials. Stockbridge (1966) employed a 
perturbation analysis and arrived at the same result as described mathematically in Equation (28). He assumed that the acoustic wave does not propagate in the film. The basic assumption made by Stockbridge is that the amount of potential energy stored in the deposited foreign layer during oscillation is negligible. This restricts the use of linear mass-frequency relationship to a small film thicknesses with negligible shear deformation (Benes, 1984). Equation 28 has been tested by Sauerbrey by evaporating thin metal films onto quartz crystals and monitoring the change in frequency (Hlavay and Guilbault, 1977).

For thick film coatings on the crystals, the assumption that acoustic waves do not propagate into the deposited film is less acceptable. Stockbridge's assumption can be justified only for very thin films. Miller and Bolef (1968) treated the quartz film combination as a composite acoustic resonator, and the deposited film was considered to be an integral part of the vibrating system. In their analysis, the acoustic waves were assumed to propagate into the deposited film. A relation for the determination of resonant frequency of the composite system was derived by Miller and Bolef:

$$
\begin{aligned}
& 2 r\left[\cos \left(\frac{2 \pi f_{c}}{f_{f}}\right)-\cos \left(\frac{2 \pi f}{f}\right)\right] \\
& +\left(1+r^{2}\right)\left[1-\cos \left(\frac{2 \pi f_{c}}{f_{q}}\right) \cos \left(\frac{2 \pi f}{f_{f}}\right)\right] \\
& +\left(1-r^{2}\right) \sin \left(\frac{2 \pi f_{c}}{f_{q}}\right) \sin \left(\frac{2 \pi f_{c}}{f_{f}}\right)=0
\end{aligned}
$$

where $r$ is the reflection coefficient of the acoustic wave at the film quartz interface and

$$
\mathrm{f}_{\mathrm{f}}=\mathrm{v}_{\mathrm{f}} / 2 \mathrm{t}_{\mathrm{f}}
$$


$v_{f}$ is the acoustic wave velocity in the film, and $t_{f}$ is the film thickness. The parameter $f_{f}$ is considered as the resonant frequency of the film if it were oscillating ( $\mathrm{Lu}$ and Czanderna, 1984). Lu and Lewis (1972) indicated that for precise mass determinations, the elastic properties of deposited material have to be taken into consideration. In the equation derived by Lu and Lewis, the resonant frequency of the composite system is determined by the acoustic properties of both the quartz crystal and the deposited film. Equation (30) can be reduced to a simpler form:

$$
\begin{aligned}
& \tan \left(\frac{\pi f_{c}}{f_{q}}\right)=-\frac{1}{Z} \tan \left(\frac{\pi f_{c}}{f_{f}}\right), \\
& Z=\frac{Z q}{Z_{f}}=\frac{\rho_{q} v_{q}}{\rho_{f} v_{f}},
\end{aligned}
$$

is the shear-mode acoustic impedance ratio between the quartz crystal and deposited film. The above expression indicates that deposited materials with different acoustic impedance's will follow different mass-frequency relations. The mass-frequency relation deviates from their linear relationship as the mass load on the crystal increases ( $\mathrm{Lu}$, 1975). The use of the above equation requires knowledge of not only the density of the coated film, but also the shear wave velocities in the film and the quartz crystal. Equation (32) is used in the current thickness monitors (Reed et al., 1990).

The explicit mass load versus frequency relation as determined by Lu and Lewis (1972) is

$$
\frac{m_{F}}{m_{Q}}=-\frac{z_{F} f_{Q}}{z_{Q} \pi f} \arctan \left(\frac{z_{Q}}{z_{F}} \tan \frac{\pi f}{f_{Q}}\right) .
$$

Equation (34) is derived by substituting 


$$
\begin{aligned}
& f_{Q}=\frac{1}{2 l_{Q}} \sqrt{\frac{c_{Q}}{\rho_{Q}}}, \\
& f_{F}=\frac{1}{21} \sqrt{\frac{c_{F}}{\rho_{F}}}, \\
& z_{Q}=\rho_{Q} v_{Q}=\sqrt{\rho_{Q} c_{Q}}, \text { and } \\
& z_{F}=\rho_{F} v_{F}=\sqrt{\rho_{F} c_{F}},
\end{aligned}
$$

in Equation (32). $v_{\mathrm{Q}}$ and $\mathrm{c}_{\mathrm{Q}}$ are the shear wave velocity and the shear stiffness constant effective in the crystal, and $v_{F}$ and $c_{F}$ are the equivalent parameters for the coated film. The validity of Equation (34) has been experimentally verified up to mass loads of $\mathrm{m}_{\mathrm{F}} / \mathrm{m}_{\mathrm{Q}} \leq 70 \%$. The latest crystal thickness monitors based on the Equations (34)-(38) are called Z-match technique devices. The only drawback of these thickness monitors is the knowledge of acoustic impedance ratio and the necessity of manually keying a $Z$ value for every deposited material (Benes, 1984).

The acoustic impedance ratio can be found approximately even in the absence of data on shear stiffness constants. In general, the density of the materials varies more strongly than the elastic stiffness constant, $c_{F}$, does, and the deviation of the specific acoustic impedance ratio $z$ from unity is primarily influenced by the square root of the density ratio,

$$
z=\frac{\sqrt{\rho_{F} c_{F}}}{\sqrt{\rho_{Q} c_{Q}}} \approx \sqrt{\frac{\rho_{F}}{\rho_{Q}}} .
$$

Quartz crystals can be used for mass determination for only small mass loading on the crystal without considering the properties of the coated materials. Since 1959, the AT 
cut quartz crystals have been widely used to monitor the thickness of vacuum deposited films. The resonator forms the frequency determining element of an electronic oscillator in these studies. The experimental observable is the frequency of the oscillator. Because the free surface of the film is at an antinode, the film deformation is negligibly small for films much thinner than the acoustic wave length and the frequency change of the resonator can be described without invoking the acoustic properties of the coated film. Sauerbrey originally realized that the frequency decrease was proportional to the mass deposited over the crystal. For small mass charges, the decrease in a thickness shear mode frequency of a crystal upon which a thin film is deposited is linearly proportional to the deposited mass. The linear mass-frequency relation is valid upto a mass load of $2 \%$ (Benes, 1984). In addition, the linear mass-frequency relation was found to be valid until the frequency decrease due to loading is approximately $2 \%$ of the unloaded frequency ( $\mathrm{Lu}, 1975)$. Beyond that range, the relation between the frequency decrease and the mass loading is nonlinear because the acoustic impedance of the coated film will be different from the acoustic impedance of quartz (Reed et al., 1990). The prediction of the maximum mass load a quartz crystal can accommodate before it fails to oscillate is difficult because it depends on many factors such as the quality of the quartz crystal, the acoustic losses in the coated material, the stresses generated in the crystal by the deposited material and the design of the crystal holder and oscillator circuitry (Lu and Czanderna, 1984).

The sensitivity of a quartz crystal is not uniform over its surface. A quartz crystal resonator with a finite size and contoured surface does not have a uniform mass 
sensitivity over its entire surface. The mass induced frequency change will depend upon the position of the deposited mass on the crystal surface. The differential mass sensitivity was found to be reaching a maximum at the center of the crystal and reduced to zero just slightly beyond the boundary of the electrode. In the equivalent circuit, the resistance increases as more material is deposited onto the crystal. The resistance increase is due to the increase in the acoustic losses in the coated material (Benes, 1984).

Three types of errors can exist in mass determination using quartz crystal microbalances. They are

1. Errors introduced from the frequency or time measurement technique.

2. Errors introduced by the frequency changes due to factors other than mass, such as temperature and stress.

3. Accuracy of the formula used to convert resonant frequency change to mass.

For periodic signals, highly accurate frequency and time (period) measuring techniques are available. Resonant frequency changes due to extraneous factors can be minimized by the proper design of the quartz crystal resonator and supporting structures (Lu and Czanderna, 1984). The oscillator circuit in the QCM should not have any influence on the resonant frequency of the crystal. The two main elements in building a proper oscillator circuit are, 
1. A wide-band oscillator which automatically adapts itself to varying crystal damping without shifting the resonance frequency of the crystal.

2. Proper circuit components which do not contribute to frequency stability when the crystals are heavily loaded.

There are two types of sensor oscillators with different stabilities. They are

1. A controlled oscillator which has variable feedback, providing a sinusoidal signal with a constant amplitude to the quartz crystal over the full damping region.

2. A fixed back oscillator which has a very strong feedback to guarantee the drive of even heavily damped crystals (Pulker et al., 1976).

\section{APPLICATIONS OF QUARTZ CRYSTAL RESONATORS}

Quartz crystals have been used in a large number of applications. Although, frequency control is a very important application of piezoelectric quartz crystals, it is certainly not the only one. The resonance frequency of a crystal is very sensitive to changes in its structure and environment. Thus, quartz resonators are used to measure temperature, pressure, thin film stresses and mass changes for scientific and environmental sensing purposes (Hafner, 1974). The sensitivity of detection is inversely 
proportional to the square of the electrode diameter and thickness of the crystal. For sorption detection, the surface must be coated evenly with the substrate. The usual amount to use is 5 to $50 \mu \mathrm{g} / \mathrm{cm}^{2}$. (King, 1964). Table I gives the references for some of the many applications involving quartz crystal resonators. These literature sources are listed to provide guidance to the interested reader in exploring other related uses of quartz crystals.

\section{STRESS EFFECTS IN MICROBALANCE APPLICATIONS}

The stress related frequency changes represent errors in the conventional microbalance applications. The elastic stiffness effects are negligible in a thin film because, there is no elastic energy stored at the surface. If the coated film is thick, the amount of elastic energy stored at the surface and the elastic stiffness of the film becomes important. In case of thin film coatings on a quartz crystal, the frequency change due to a mass change can be represented by,

$$
\frac{d f_{m}}{f}=-\frac{m_{f}}{t_{q} \rho_{q}}=-\frac{\rho_{f} t_{f}}{\rho_{q} t_{q}}
$$

where $m_{f}$ is the areal mass density of the film, $\rho_{q}$ and $\rho_{f}$ are densities of quartz and the film respectively and $t_{q}$ and $t_{f}$ are the thicknesses of the crystal and film respectively. If uniform stress is assumed throughout the thickness of the film

$$
\mathrm{S}_{\mathrm{f}}=\mathrm{T}_{\mathrm{f}} \mathrm{f}
$$

The relative effects of stress and mass in a thin film on the surface of the quartz resonator can represented by 
Table I.

Applications of Quartz Crystals and References

\begin{tabular}{|c|c|}
\hline Application & Reference \\
\hline Measuring Sputtering & McKeown (1961) \\
\hline Air Pollution Detection & Scheide, Guilbalt, and George (1972) \\
\hline Sorption Detection & $\begin{array}{l}\text { King (1964); King (1969a); King (1969a); King and } \\
\text { Corbett (1969); Xu and Schenoff (1994); Quist, Huth- } \\
\text { Fehre, and Sundqvist (1994); Utada et al (1991); } \\
\text { Laatikainen and Lindstrom (1986); Bonner and Chang } \\
\text { (1975). }\end{array}$ \\
\hline Solubility Measurements & Moylan, Best, and Ree (1991) \\
\hline Analytical Chemistry & $\begin{array}{l}\text { Hlavay and Guilbault (1977); Carey and Kowalski (1986); } \\
\text { King (US patent 3,164,004); Fischer and King (1967). }\end{array}$ \\
\hline $\begin{array}{l}\text { Measuring Oxidation } \\
\text { Stability of Elastomers }\end{array}$ & Panjan, Zabkar, and Navinsek (1985) \\
\hline Depth Profiling & Zabarnick and Grinstead(1994) \\
\hline Thermocouples & Tanaka et al. (1994) \\
\hline $\begin{array}{l}\text { Studying Jet Fuel } \\
\text { Additives }\end{array}$ & Karasek and Gibbins (1971) \\
\hline Mass Detection & Ward and Buttry (1990) \\
\hline
\end{tabular}


Table I.

Applications of Quartz Crystals and References (Continued)

\begin{tabular}{|l|l|}
\hline Chromatography & $\begin{array}{l}\text { Schulz and King (1973); Konash and Bastiaans (1980); } \\
\text { Karasek et al. (1976); Karasek and Tiernay (1974); King, } \\
\text { Camilli and Findeis (1968) }\end{array}$ \\
\hline Electrochemical & $\begin{array}{l}\text { Deakin and Byrd (1989); Lee, White and Ward (1993); } \\
\text { Bilger and Heinze (1991); Hammer, Benes and Pulker } \\
\text { (1976) }\end{array}$ \\
\hline Pharmaceutical & $\begin{array}{l}\text { Kurosawa et al. (1990); Wanzhi et al. (1993); Muratsugu et } \\
\text { Applications }\end{array}$ \\
\hline
\end{tabular}




$$
\frac{\mathrm{df}_{\mathrm{s}}}{\mathrm{df}_{\mathrm{m}}}=\frac{\mathrm{KT}_{\mathrm{f}} \rho_{\mathrm{q}}}{\rho_{\mathrm{f}}}
$$

The above relation can be evaluated if the stress levels in the thin film are known. The stress level is highest for the first few monolayers of a thin film deposited on the substrate, but then decreases as subsequent monolayers are deposited. The frequency change due to stress effects can be very large compared to the mass related frequency changes.

For example, for ion bombardment of Ar atoms on a quartz crystal

$$
\frac{d f_{s}}{d f_{m}}=-552
$$

Correction of the frequency changes to account for stress-related effects can be done if the actual stress levels are known. If the frequency changes due to the mass change and due to the stress effects are considered as linear perturbations, the total observed frequency change $\mathrm{df} / \mathrm{f}$ is given by,

$$
\frac{d f}{f}=\frac{d f_{s}}{f}+\frac{d f_{m}}{f} .
$$

If $\mathrm{S}_{\mathrm{f}}$ is measured with the cantilever beam technique or any other stress measuring technique, the frequency can be corrected by the following relation

$$
\frac{d f}{f}=-\frac{K S_{f}}{t_{q}}-\frac{M_{f}}{\rho_{q} t_{q}}
$$

No correction is possible unless the stress levels are known.

An SC cut quartz crystal can be used as a sensor in applications to eliminate stress effects and thermal shock. The stress-compensated cut of the quartz (i.e., the SC-cut) 
provides good resistance to thermal shock and stress effects. The SC-cut is a doublyrotated cut, whereas the AT-cut and BT-cut are singly rotated cuts. In the manufacture of a SC-cut quartz crystal, the quartz plate is rotated about two of the crystallographic axes before cutting (Bottom, 1982). The use of the second rotation of quartz crystal, when cut from a larger quartz crystal, provides an additional degree of freedom so that stress effects and temperature effects can be minimized. The SC cut crystal has electrical characteristics similar to those of AT-cut and BT-cut crystals. Retrofitting the SC-cut crystal into an existing microbalance application should not be difficult. The only problem that could arise with SC-cut crystal is that the fast shear wave mode, called the B mode, of the resonator is closer in frequency to the desired slow shear wave mode than in AT-cut or BT-cut resonators, so some circuits may cause oscillation at the wrong frequency (Lu and Czanderna, 1984).

\section{VISCOELASTIC EFFECTS IN MICROBALANCE APPLICATIONS}

In addition to the frequency decrease due to the deposition of mass onto crystal surface, it has been found that the amplitude of vibration of the crystal reduces slightly due to the deposition of the film. The damping effect can be due to the viscous effects in the deposited film and at the interface between the deposited film and the quartz crystal. Some of the other factors which influences the damping are, the mechanical properties of the coated films and slippage between the deposited films and the quartz crystal. A linear relation exists between the amplitude change and the mass of the deposited film. The expression can be empirically expressed as, 


$$
\frac{\Delta \mathrm{A}}{\mathrm{A}_{\mathrm{o}}}=-\mathrm{c} \frac{\Delta \mathrm{m}}{\mathrm{m}_{\mathrm{q}}},
$$

where $A_{o}$ is the non-damped amplitude of the quartz crystal, $m_{q}$ is the mass of the crystal, $\Delta \mathrm{A}$ is the amplitude change, $\Delta \mathrm{m}$ is the mass of the deposited film and $\mathrm{c}$ is a constant. The value of $\mathrm{c}$ depends on the deposited film. Fluid like materials show a larger damping effect than rigid materials. The decrease in crystal vibration amplitude has been recognized to be due to the energy dissipated within the deposited film. A model called the Energy Transfer Model (ETM) (Mecea and Bucur, 1979) has been proposed to include the effect of mechanical losses in the deposited film resulting in the damping of the crystal. In this model, the quartz crystal and deposited film form a compound resonator. The active portion of the compound resonator is assumed to be the quartz crystal itself and it is also assumed that the vibration of the deposited film is caused by the energy stored in the quartz crystal vibration. According to the ETM model, when a film is deposited on the crystal surface, some of the energy stored in the quartz crystal vibration is transferred to the film. The transferred energy is shared between the stored energy and dissipated energy within the film. The distribution of the energy as stored and dissipated portions is in accordance with the elastic properties of the deposited film.

The deposited film is assumed to be oscillating synchronously with the quartz crystal surface in the ETM model (Mecea and Bucur, 1979). In the case of a coated, undamped crystal, the reduction of frequency on coating the crystal is due to the transfer of energy to the deposited film as stored energy, proportional to the mass of the film and with no energy dissipation in the film (Mecea et al., 1989). The crystals vibrations will 
be completely damped, if all the vibrational energy of the quartz crystal is transferred to the deposited film mainly as dissipated energy. This can happen if the crystal is coated with an viscous film of sufficient thickness or if the stresses in the deposited film to cause peeling of the film (Mecea and Bucur, 1979). The vibration of the quartz crystal stops at a certain loading on the surface and the limiting mass is dependent on the mechanical rigidity of the deposited film. The damping characteristics depend upon the amount of vibrational energy dissipated in the film (Mecea and Bucur, 1974).

The ETM model was contradicted by Kanazawa (1990). He indicated that, in an elastic solid, energy is stored in the deformation of the solid and is completely recoverable. He also indicated that, in a purely viscous fluid, no recoverable energy can be stored in the form of a deformation of the fluid. Kanazawa's hypothesis was contradicted by Mecea (1990), that a deposited viscous fluid has an interface with a vibrating solid and must be treated as a viscoelastic fluid. Mecea and Bucur and did not consider the effect of surface morphological effects in their ETM model for explaining the damping in coated crystals (Yin and Collins, 1995). Yin and Collins (1995) had studied the effect of morphology of thin films on the damping of quartz crystals. The damping of a quartz crystal is monitored by measuring the amplitude of the driving voltage of the oscillator circuit. The amplitude of the signal is rectified and converted to a dc value for their analysis. The dc amplitude of a quartz crystal oscillator is believed to be proportional to the amplitude of mechanical vibration of the quartz crystal. Yin and Collins (1995) showed that the damping effect is strongly dependent on the morphology of the films. Unfortunately, no quantitative model has been proposed for the dependence 
of damping on morphology and viscous properties, and further study is required in this area (Yin and Collins, 1995).

The linear relationship between the mass loading on quartz crystals and the frequency change is found to be only valid for thin elastic films. In the derivation of this linear relation, the free surfaces of the resonator were assumed to be at the antinodes of vibration. In the case of a thin film having thickness negligible compared to the wavelength of the shear wave, the resonant frequency of the resonator is independent of the acoustical properties of the coated film. As the film thickness increases, an increasing fraction of the shear wave is occupied by the film. So the quartz and film must be considered as a compound resonator (Kanazawa and Melroy, 1993). The Sauerbrey relation, Equation (28) was found to be valid up to a frequency change of about $2 \%$ of the uncoated crystal frequency. Beyond that range the analytical expression developed by Lu and Lewis (1972) must be used.

The quartz crystal is sensitive to both the viscosity and elasticity of the coated films. If the coated film is viscoelastic, the resulting frictional dissipation in the viscoelastic film absorbs energy from the shear waves. Losses in the film can considerably change the behavior of the quartz crystals (Kanazawa and Melroy, 1993). One solution to the problem would to use an impedance analyzer to measure the shape of the resonance rather than the frequency shift of the crystals. This technique would be useful to study the behavior of even the heavily loaded crystals which cannot support the oscillations (Reed et al., 1990). This technique is not yet been fully developed. 
The operation of a quartz crystal in contact with a different fluid can be described with two different models. Both the models were described by Kanazawa and Melroy (1993). In the first model which is called the mechanical model, the electrical properties of quartz are not included in the analysis. The resonance is treated purely as mechanical resonance induced by constructive interference of the shear waves in quartz crystal and in the coated film. The solution of this model equations only describes the resonant frequency. In the second model called the complete model, a more general and complete solution was obtained by including the electrical properties of the quartz crystal. The solution of this model gives the fundamental resonant frequency, electrical admittance and also the overtone resonant frequencies. This model has the potential to determine the effect of mechanical properties of the coated film on the resonant frequency. The frequency shifts of the resonators in contact with several fluids have been observed experimentally. The observed shifts were found to be consistent with the model predictions.

Experiments were performed by Reed et al. (1990) on very viscous fluids such as perfluoropolyethers to test the behavior of quartz crystals on viscous loading. Serious discrepancies were observed between the predictions of the complete model and observed shifts. A series of fluids with varying molecular weights were studied. The difference between the predicted and the observed frequency shifts increased with increasing molecular weight and also with increasing viscosity of the liquids. These discrepancies were resolved to a certain extent by considering the liquids as viscoelastic Maxwellian fluids instead of Newtonian fluids. Before observing these discrepancies, the fluids in 
contact with the crystals were assumed to be Newtonian fluids. In a Maxwellian fluid model, the shear modulus and the viscosity of the fluid were assumed to be dependent on the frequency of oscillation. After these modifications were made, the predicted values of frequency from the complete model were improved but were still only in semiquantitative agreement with the observed values. More quantitative work must be done in this area to fully understand the behavior of quartz crystals loaded with viscoelastic films (Kanazawa and Melroy, 1993 and Yin and Collins, 1995). These studies indicate that the operation of crystals under viscoelastic loading is not yet fully understood and more study needs to be done in this area.

\section{PENETRANT DIFFUSION IN POLYMERS}

\section{BACKGROUND TO PENETRANT-POLYMER DIFFUSION}

Knowledge of the rate of diffusion of small molecules in polymers is essential in the design of numerous processes and applications including devolatilization, medicinal time controlled release, drying of paintings and coatings, packaging or barrier membranes and separation processes. Recently, the transport behavior of small molecules in polymers has been of primary focus in industry due to the increase of emphasis on reduction of emissions from commercial processes. However little data of these type are available in the literature, particularly at varying solvent concentrations. So, acquiring polymer-penetrant transport data are extremely important.

The fundamental physical property that describes molecular mass transfer coefficient is the mutual diffusion coefficient. In systems with low molecular weight 
materials, the mutual diffusion coefficients are weak functions of concentration and temperature. If one of the species is a macromolecule, diffusion coefficients are very strong functions of temperature and concentration and also cover a wide range of magnitude. In addition, the diffusion process in polymers sometimes does not follow the classical laws of diffusion (i.e., Fick's Law). An understanding of these characteristics is necessary for the development of detailed analyses of mass-transfer problems involving polymer-penetrant systems (Vrentas and Duda, 1986).

When a penetrant diffuses into a polymer, the polymer molecules rearrange to a new conformation that is consistent with the concentration of the penetrant. The relative rates of diffusion of the penetrant and relaxation of polymer molecules determine the nature of the diffusion process. Comprehensive analysis of polymer-penetrant diffusion process is possible when the diffusion process can be described by classical theories of diffusion. Fick's law is the constitutive equation for describing the mutual diffusion coefficient in an unambiguous manner. Detailed analyses of viscoelastic and anomalous mass transport processes in polymer-penetrant systems is not possible with the present state of knowledge. Anticipation of the type of transport process involving mass transfer of a solvent in an amorphous polymer is important. A dimensionless group called the diffusion Deborah number provides a means of characterizing various types of diffusion (Vrentas and Duda, 1986).

Anomalous effects in polymer-penetrant diffusion can be anticipated by comparing the rate of diffusion of the penetrant with the rate of rearrangement of polymer 
molecules. A diffusion Deborah number is defined as the ratio of a characteristic time $\lambda_{m}$ of the fluid to the characteristic time $\theta_{D}$ of the diffusion process,

$$
(\mathrm{DEB})_{\mathrm{D}}=\frac{\lambda_{\mathrm{m}}}{\theta_{\mathrm{D}}} .
$$

The parameter $\lambda_{\mathrm{m}}$ is a strong function of temperature and concentration for a polymersolvent mixture. The transient diffusion of the solvent in the polymer film can be described by a single value of the diffusion Deborah number, only if the difference in the final and initial equilibrium concentrations is small. This type of experiment is called differential sorption experiment. For sorption experiments, where large changes in concentration occur, the nature of diffusive transport can be obtained by calculating Deborah numbers at the initial and final conditions of diffusion process (Vrentas and Duda, 1986).

The characteristic time for a polymer-solvent system is defined as,

$$
\lambda_{m}=\frac{\int_{0}^{\infty} t G(t) d t}{\int_{0}^{\infty} G(t) d t},
$$

where $G(t)$ is the shear relaxation modulus for the system (Vrentas and Duda, 1979). The characteristic time $\theta_{D}$ of the diffusion process must be defined for each mass-transfer operation. The temperature and concentration dependence of $\lambda_{m}$ can be determined from experimental data of the polymer-solvent system. For a step-change sorption experiment involving diffusion of a penetrant into a polymer film of initial thickness $\mathrm{L}$, an average characteristic time for one dimensional mass-transfer can be defined as 


$$
\theta_{\mathrm{D}}=\frac{\mathrm{L}^{2}}{\mathrm{D}^{*}}
$$

where $\mathrm{D}^{*}$ is the mutual diffusion coefficient.

$$
\mathrm{D}^{*}=\mathrm{x}_{2} \mathrm{D}_{1}+\mathrm{x}_{1} \mathrm{D}_{2}
$$

where $D_{1}$ and $D_{2}$ are the self diffusion coefficients and $x_{1}$ and $x_{2}$ are the mole fractions of solvent and polymer, respectively (Vrentas et al., 1975). Self diffusion coefficients can be estimated for a sufficiently concentrated polymer solutions using the free-volume theory of diffusion (Vrentas and Duda, 1977).

Penetrant diffusion in polymers can be characterized by calculating the diffusion Deborah number. When the diffusion Deborah number is small $(<0.1)$, the molecular relaxation of the polymer is much faster than the diffusive transport. Conformational changes in the polymer structure appear to take place instantaneously, and the diffusion process involves essentially a purely viscous binary mixture. This process is called viscous diffusion. When the diffusion Deborah number is large $(>10)$, there is essentially no time variation of the structure of the polymer during the diffusion process. Since a diffusing penetrant molecule is moving in a material that effectively has the properties of an elastic solid, this type of diffusional transport is called elastic transport. If (DEB $)_{D}$ is of the order of unity, the molecular relaxation of the polymers and diffusive transport occur on comparable time scales. This type of diffusion is called viscoelastic diffusion (Vrentas and Duda, 1986).

When the polymer-solvent system behaves like a purely viscous fluid, the diffusion process can be described by the classical laws of diffusion. An elastic diffusion process can be analyzed using the classical diffusion equation with a diffusion coefficient 
independent of concentration even though the system is not a purely viscous mixture. From the experimental data for low penetrant concentrations below the glass transition temperature of the polymer where the diffusion Deborah number is very high, the elastic diffusion process can be considered to be Fickian even though no definitive theoretical justification is available. Calculation of the Deborah number can indicate the type of diffusion that can be expected. Anomalous effects in polymer-solvent diffusion can be anticipated by the calculation of the diffusion Deborah numbers (Vrentas and Duda, 1986).

When the diffusion Deborah number is of the order of unity, the diffusion process can be called as viscoelastic and the mass transfer process cannot be explained by the classical laws of diffusion. Diffusion of organic vapors in glassy polymers show significant deviation from the Fickian behavior (Vrentas and Duda, 1986). Different types of anomalous behavior have been observed under viscoelastic diffusion. They are

1. Thickness anomalies.

2. Anomalous sorption curves

3. Case-II sorption

4. Solvent crazing and swelling fracture

5. Dual mode sorption

More detailed description of the above processes is given by Duda and Vrentas (Vrentas and Duda, 1986). 


\section{CLASSICAL EXPERIMENTAL METHODS FOR PENETRANT-POLYMER}

\section{DIFFUSION}

In a typical permeation experiment, a dry polymer film is inserted between two completely evacuated chambers. Vapor is suddenly introduced at a given pressure into one of the chambers and the amount of vapor permeating the film is measured as a function of time. The permeation experiments are practical only for relatively low solvent concentrations. Permeation experiments are particularly useful for the measurement of diffusivity of penetrants with a low solubility in the polymer.

Conventional methods for measuring diffusion coefficients become extremely difficult to apply to polymer-solvent systems at infinite dilution of the solute (i.e., when the solvent is present in extremely small amounts). In recent years, a new method called the Inverse Gas Chromatography (IGC) has been developed for studying thermodynamic and transport properties in concentrated polymer solutions. In a typical IGC experiment, the solute is injected at the column inlet as a plug of vapor and the solute is swept through the column by the carrier gas. As the solute migrates down the column, the solute band broadens due to mass transfer resistances. The most common contributions to peak spreading are longitudinal diffusion in the gas phase and mass transfer resistance in the polymer phase. This peak dispersion has been used as a means of measuring diffusion coefficient of a low molecular weight penetrants in polymers at infinite dilution concentration of penetrants (Hadj Romdhane, 1994).

Sorption experiments are some of the most widely used experimental methods for measuring the solubility and diffusivity of solvents in polymers. In a typical step change 
sorption experiment, a uniform film of a polymer is suddenly exposed to the penetrant atmosphere and the gain or loss of film weight is measured as a function of time. Information on the diffusion coefficient is obtained from the sorption curve (Vrentas and Duda, 1986).

Sorption techniques have several advantages over the permeation techniques. In permeation studies, the efficiency of sealing of the membrane is very important. But in sorption techniques it is not a factor. Small holes in the permeating membrane greatly affect the permeation rate, but have little affect in sorption studies. The sorption technique is valuable in the case of long experiments required for low diffusion coefficients. Errors due to leakage and distortion are significant in permeation experiments (Crank and Park, 1968)

Some of the other techniques used for the measurement of diffusion coefficients in polymers are

1. Refractive Index Techniques

2. Radiation Absorption Methods

3. Radiotracer Methods

4. Nuclear Magnetic Resonance Techniques.

More detailed descriptions of the above methods are available in Crank and Park (1968).

\section{ANALYSIS OF SORPTION EXPERIMENTS}

In analysis of the recorded sorption data from the quartz crystal microbalance, the following assumptions are made. 
1. There is no temperature change associated with the diffusion.

2. Diffusion is mainly occurring in one dimension.

3. Pressure has negligible effect on the polymer phase.

4. Negligible volume change on mixing.

5. The polymer is assumed to be a isotropic substance.

6. Constant diffusivity is assumed.

The first assumption is valid since an analysis by Crank (1957) indicated that the change in polymer surface temperature accompanying the sorption process is less than $0.25^{\circ} \mathrm{C}$. The second assumption is valid because the thickness of the polymer sample is much smaller compared to the area of the coated polymer film on the quartz crystal. Fick's law is further simplified by assuming constant diffusivity. In the actual case, the diffusivity is dependent on concentration. If the experiment is performed so that the concentration changes by a small amount during the sorption experiment, the diffusion coefficient obtained will be some average value over that range of concentration. In practice this is done by introducing small amounts of penetrant into the chamber for a given sorption run. Vrentas et al. (1977) analyzed the step change sorption experiments and concluded that the average diffusion coefficient obtained from step-change sorption experiments represents the actual diffusivity at a concentration which lies at 0.7 of the concentration interval. The error associated with this assumption was shown to be less than 5 percent. 
Under the experimental conditions with the assumptions given above, the general equation describing the one-dimensional diffusion becomes

$$
\frac{\partial \mathrm{C}}{\partial \mathrm{t}}=\mathrm{D} \frac{\partial^{2} \mathrm{C}}{\partial \mathrm{x}^{2}},
$$

with the associated initial and boundary conditions:

$$
\begin{aligned}
& C(x, 0)=C_{0}, \\
& C(L, t)=C_{E} \text {, and } \\
& \frac{\partial}{\partial x}(0, t)=0 .
\end{aligned}
$$

The first boundary condition is obtained from the assumption that the concentration of solvent at the surface, $\mathrm{x}=\mathrm{L}$, is at its equilibrium value $\mathrm{C}_{\mathrm{E}}$. The second boundary condition is obtained from the assumption that there is no transport of penetrant through the bottom of the sample at $x=0$. The exact solution of the above set of equations is given by Crank (1957). The concentration profile must be integrated to get the weight of the penetrant absorbed by the polymer.

The solution of the above set of equations in terms of mass uptake is (Crank, 1957):

$$
\frac{M(t)}{M(\infty)}=2\left[\frac{D t}{L^{2}}\right]^{1 / 2}\left[\frac{1}{\sqrt{\pi}}+2 \sum_{n=1}^{\infty}(-1)^{n} \operatorname{erfc}\left(\frac{n L}{(D t)^{1 / 2}}\right)\right],
$$

where

$$
\begin{aligned}
& M(t) \quad=\text { weight pickup at time } t \\
& M(\infty)=\text { weight pickup at infinite time (i.e., equilibrium), } \\
& D \quad=\text { diffusivity, and }
\end{aligned}
$$




$$
\mathrm{L} \quad=\text { thickness of the sample. }
$$

At small values of time, the summation term in the above equation can be neglected and the above equation can be written as

$$
\frac{M(t)}{M(\infty)}=\frac{2}{\sqrt{\pi}}\left[\frac{D t}{L^{2}}\right]^{1 / 2}
$$

Weight pickup versus $\sqrt{\mathrm{t}}$ is recorded from the QCM experiments. The average diffusion coefficient can be calculated as

$$
\mathrm{D}=\frac{\pi}{4} \mathrm{R}_{1}^{2} \mathrm{~L}^{2}
$$

where

$$
\begin{aligned}
& L=\text { thickness of the polymer sample, and } \\
& R_{1}=\text { Initial slope of } \frac{M(t)}{M(\infty)} \text { vs } \sqrt{t} \text { curve. }
\end{aligned}
$$

The diffusion coefficient calculated in the above manner is an average diffusion coefficient corresponding to an average concentration, $\bar{\omega}$, at 0.7 of the concentration interval. By monitoring the weight uptake by the polymer during the sorption run, a reduced sorption curve can be constructed. A plot of $\frac{M(t)}{M(\infty)}$ vs $\sqrt{t}$ is called as a reduced sorption curve. Since the frequency change of the piezoelectric quartz crystal is proportional to the change of mass on the crystal, the ratio of mass at time $t$ to the mass at equilibrium can be found from

$$
\frac{M(t)}{M(\infty)}=\frac{C\left[f_{2}(t)-f_{1}\right]}{C\left[f_{2}(\infty)-f_{1}\right]}=\frac{f_{2}(t)-f_{1}}{f_{2}(\infty)-f_{1}}
$$


where $\mathrm{C}$ is the proportionality constant, $\mathrm{f}_{2}(\mathrm{t})$ is the frequency of the crystal at time $\mathrm{t}$ as the penetrant diffuses into the polymer coating, and $f_{1}$ is the frequency of the polymer-coated solvent-free crystal. The equilibrium weight fraction of the solvent sorbed by the polymer, $\omega$, can be calculated by

$$
\omega=\frac{f_{2}(\infty)-f_{1}}{f_{2}(\infty)-f_{o}}
$$

where $f_{o}$ is the frequency of the bare, uncoated crystal. The activity of the penetrant can be readily determined from the pressure of the penetrant vapor in the chamber, $\mathrm{P}$, and the vapor pressure of the penetrant at the temperature of the vacuum chamber, $\mathrm{P}^{*}$, since the chamber is typically operated below the atmospheric pressure. The average weight fraction can be calculated as

$$
\bar{\omega}=\omega(\text { initial })+0.7 *[\omega(\text { final })-\omega(\text { initial })],
$$

where

$\bar{\omega}=$ Average weight fraction of the solvent sorbed by the polymer film.

\section{FREE VOLUME MODEL FOR POLYMER-PENETRANT DIFFUSION}

At present, there is no general theory which can describe the complete range of the complex diffusional behavior exhibited by polymer-solvent systems. Very little progress has been made in the formulation of theories which describe the anomalous mass transport behavior in polymer-solvent systems. Until recently, no comprehensive theory was available for the prediction of mutual diffusion coefficients for polymersolvent systems for conditions under which classical diffusion theory is applicable. In 
concentrated systems, theories based on free-volume concepts are the most useful for predicting and correlating diffusivity data (Duda, 1983).

The volume of a liquid can be divided into two parts. There is a volume that is directly occupied by the molecules. The unoccupied space between the molecules is referred to as the free volume. Due to random thermal fluctuations, some of this free volume is continuously being redistributed causing fluctuations in the local density. This part of the free volume is referred to as the hole free volume. Molecular transport in the hole free volume occurs by the migration of molecules into these randomly fluctuating voids. The remaining free volume which is not being continuously redistributed and is not available for molecular migration is referred to as the interstitial free volume. The amount of free volume and the size of the migrating molecule determines the rate of diffusion. According to free volume theory, two criteria should be satisfied for a molecule to migrate A free-volume hole of sufficient size must occur and, also the molecule must have enough energy to jump into this hole. Therefore, the rate of migration can be considered to be a product of two probabilities: the probability that a hole of sufficient size will occur and the probability that the molecule will have the required activation energy to jump into the hole. For ordinary liquid systems, both the probabilities should be taken into account for predicting molecular diffusion. If a system is close to a glassy state, the available hole free volume is very small and so, the probability that a hole of sufficient size will occur dominates the migration process. The availability of free volume dominates the molecular transport up to temperatures of 100 
${ }^{\circ} \mathrm{C}$ above the glass transition temperature. The free volume parameters obtained from viscosity data can be then be used to predict diffusion coefficients (Duda, 1983).

Free volume increases with temperature and free volume theory predicts that the diffusion coefficient increases with temperature. The larger a system free volume, the farther the system is away from its glassy state. Solvents contain large quantities of free volume and the diffusion of a solvent into a polymer will greatly increase the free volume of the mixture. Because of the increase of free volume of the mixture, the diffusion coefficient will increase with the solvent concentration.

According to the free volume theory most solvents migrate so that the complete molecule jumps as a unit. The polymer molecule moves through the solution by the coordinated movement of segments of the chain. The critical volume for migration of a polymer chain is the volume of this jumping unit which is only a segment of the complete polymer chain (Duda, 1983).

Free volume theory developed by Zelinski and Duda (1992) is the only known technique that predicts the polymer/solvent diffusion behavior without the use of any experimental diffusion data. Zelinski and Duda (1992) derived the expressions for calculation of binary polymer/solvent mutual diffusion coefficient from the free volume theory. The expressions are given in equations (61) and (62).

$$
\begin{aligned}
& D_{1}=D_{0} \exp \left(\frac{-E}{R T}\right) * \\
& \exp \left[\frac{-\left(\omega_{1} V_{1}^{*}+\omega_{2} \xi V_{2}^{*}\right)}{\omega_{1}\left(\frac{K_{11}}{\gamma}\right)\left(K_{21}-T_{g 1}+T\right)+\omega_{2}\left(\frac{K_{12}}{\gamma}\right)\left(K_{22}-T_{g 2}+T\right)}\right]
\end{aligned}
$$




$$
\begin{gathered}
\text { and } \\
\mathrm{D}=\mathrm{D}_{1}\left(1-\phi_{1}\right)^{2}\left(1-2 \chi \phi_{1}\right)
\end{gathered}
$$

The solvent parameters are subscripted 1 and polymer parameters are subscripted $2 . \hat{\mathrm{V}}_{1}^{*}$ is the specific critical hole free volume of component 1 required for a jump, $\omega_{1}$ is the mass fraction of component $1, \mathrm{~T}_{\mathrm{g} 1}$ is the glass transition temperature of component $1, \phi_{1}$ is the solvent volume fraction, and $\chi$ is the polymer-solvent interaction parameter. $\mathrm{D}_{\mathrm{o}}$ is a constant pre-exponential factor, $\mathrm{E}$ is the energy per mole that a molecule needs to overcome attractive forces which constrain it to its neighbors, and $\gamma$ is an overlap factor (between 0.5 and 1) introduced because the same free volume is available to more than one molecule. $\mathrm{K}_{11}$ and $\mathrm{K}_{21}$ are free-volume parameters for the solvent, while $\mathrm{K}_{12}$ and $\mathrm{K}_{22}$ are free-volume parameters for the polymer. The parameter $\xi$ is the ratio of molar volumes for the solvent and polymer jumping units (Duda, 1983). There are 13 independent parameters to be evaluated in Equation 61. Grouping some of them leaves 10 parameters to be determined to estimate the mutual diffusion coefficients: $\mathrm{K}_{11} / \gamma, \mathrm{K}_{21^{-}}$ $\mathrm{T}_{\mathrm{g} 1}, \mathrm{~K}_{12} / \gamma, \mathrm{K}_{22}-\mathrm{T}_{\mathrm{g} 2}, \hat{\mathrm{V}}_{1}^{*}, \hat{\mathrm{V}}_{2}^{*}, \chi, \mathrm{D}, \mathrm{E}$ and $\xi$. All of these parameters have physical significance and they are not simply the adjustable parameters which allow for good empirical fits to the data (Zelinski and Duda, 1992).

Equation 62 contains the implicit assumptions that (1) the mutual diffusion coefficient is related theoretically to the solvent and polymer self diffusion coefficients, (2) the contribution of the polymer self-diffusion coefficient to the mutual diffusion coefficient is negligible, and (3) the Flory-Huggins model accurately describes the 
penetrant activity. The basic FV model (Equation 62) can be easily modified if a more suitable thermodynamic expression becomes available and the self/mutual diffusion coefficient coupling is explained. The specific free-volumes of the polymer and solvent are presumed to be additive (without a volume change on mixing) and thermal expansion coefficients are approximated by average values over the temperature intervals of interest, thereby limiting the range of applicability of the model (Zelinski and Duda, 1992).

\section{EQUILIBRIUM SORPTION MODELS}

All the models developed for the prediction of polymer solution properties can be classified into two categories: lattice models and van der Waal models. In both the lattice models and van der Waal models, the behavior of molecules can be described as the sum of two contributions. In the first contribution only the size and shape of the molecules are considered. In this contribution, the intermolecular interactions were assumed to be absent. The interaction-free contribution is generally called the combinatorial term or the athermal term. In the case of van der Waal models, this term is called as the free volume term. The second contribution is assumed to originate from intermolecular interactions. This contribution is referred to as the attractive energy term, the residual term or the potential energy term. In this study the experimental data are compared with three different models. The software from Danner and High (1993) has been used to calculate the equilibrium sorption predictions from all the three models.

Oishi and Prasunitz (1978) model is the modification of the highly successful Universal Functional Group Activity Model (UNIFAC). Oishi and Prasunitz (1978) 
included the contribution of free volume differences between the polymer and solvent molecules in their model. This model is one of the many lattice models available for the prediction of polymer solution properties.

The High-Danner equation of state model is also a lattice model. High and Danner (1990) used a group contribution approach for the determination of molecular parameters in their model. The High-Danner model has the capability of predicting polymer-solvent equilibria from the structure of polymer and solvent molecules.

The model developed by Chen et al. (1990) is a van der Waal model and is a modification of Flory equation of state. The Chen et al. equation of state is not as accurate as the other two models described above. The Chen et al. equation of state has the advantage of not requiring pure component densities. The densities of pure components and mixtures are calculated through a group contribution approach (Danner and High, 1993).

Chapter II summarizes the previous research relevant to the operation of quartz crystal microbalances. Chapter II also summarizes the relevant research work in polymer-penetrant diffusion. 


\section{CHAPTER III}

\section{EXPERIMENTAL APPARATUS}

\section{A. EXISTING SETUP}

The objective of this work was to modify and use the existing piezoelectric quartz crystal microbalance apparatus developed by Deshpande (1993) to measure the diffusion characteristics of a penetrant into a polymer film. A schematic diagram of the QCM is shown in Figure 5. The detailed stepwise experimental procedure is given in the Appendix 1. The polymer film was subjected to a pure penetrant atmosphere with an extremely low level of contaminants. The experiment was conducted in a high vacuum chamber so that minimal contamination was present.

The previously built Quartz Crystal Microbalance (QCM) had the following characteristics. A high vacuum six-way cross was used as the experimental chamber. The chamber was operable from $10^{-11}$ torr to slight positive pressures and up to a maximum temperature of $500{ }^{\circ} \mathrm{C}$. A standard crystal holder was used as a sensor for measuring the resonant frequency of the quartz crystal. The crystal holder has a water circulation facility. A standard quartz crystal thin film deposition controller was used to monitor the frequency of the quartz crystal as the penetrant diffuses into the polymer. The deposition monitor was interfaced to the computer using an RS232 port. A computer program records the data at regular intervals of time. The system was enclosed in a 


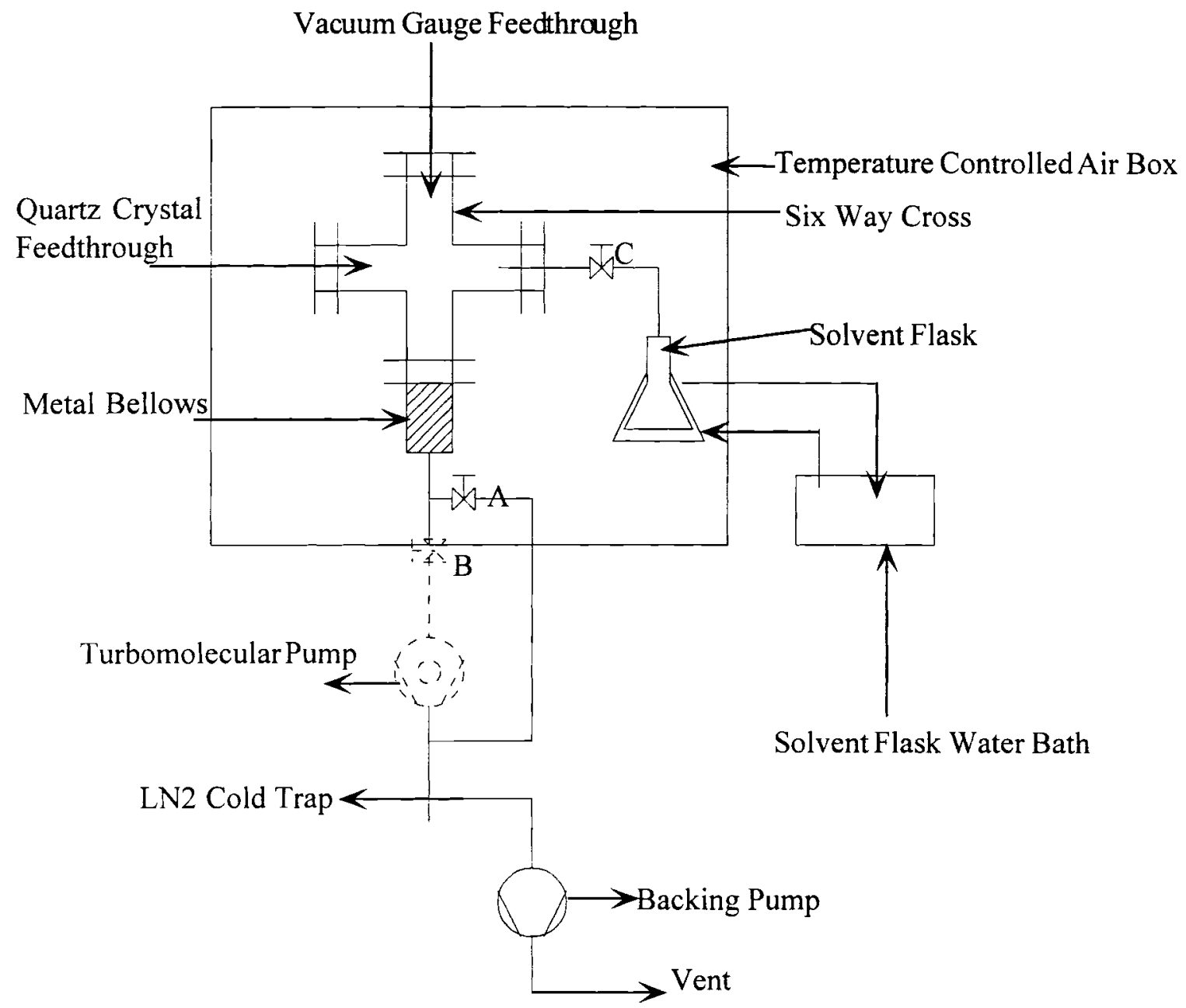

ThermocoupleFeedthrough and Viewport are not shown

Figure 5.

Schematic Diagram of the Apparatus 
thermal box made of plywood lined with insulating material to accurately control the chamber temperature and thereby control the polymer film temperature. A heating element mounted at the outlet of a circulating fan was used to circulate hot air in the chamber. A temperature controller was used to control the temperature inside the thermal box. The constant temperature around the chamber aids in the accurate control of the temperature of the coated polymer film.

To maintain the chamber free of contamination, the system was evacuated using a rotary vane pump. Pressures down to $10^{-3}$ torr were reached using the rotary vane pump. A liquid nitrogen trap was used to prevent the backstreaming of oil vapors of the pump into the chamber and to prevent the contamination of the oil in the rotary vane pump by solvent vapors while evacuating the chamber. A glass flask containing the penetrant was connected to the chamber using $1 / 4$ inch stainless tubing. A toggle valve was used to control the flow of penetrant into the chamber. A needle valve was present in the piping to isolate the flask and vent the system to the atmosphere. The flask containing the penetrant was kept in a water bath heated with a constant temperature immersion circulator to keep the penetrant at the desired temperature.

A Pirani gauge and an hot-cathode ionization gauge were connected to the chamber for the accurate measurement of chamber pressure. The Pirani and ionization gauge were used to measure low and high vacuum, respectively. They were also used to measure the extent of vacuum in the chamber. A resistance temperature device was connected to the chamber to measure the temperature inside the chamber. 


\section{B. EQUIPMENT MODIFICATIONS}

Several modifications were made to the apparatus during this study to prevent operational difficulties, to increase the accuracy of the data collected and to increase the ease of operation of the apparatus.

A new pressure gauge from Druck Instruments (Model DPI 265) was connected to chamber to accurately measure the chamber pressure when the penetrant was introduced into the chamber. This gauge can be used to measure the pressure due to any solvent vapors in the chamber in the range of 0-19.999 Psia. This gauge can also withstand temperatures up to $300{ }^{\circ} \mathrm{C}$. The previously installed Pirani gauge and ionization gauge were not accurate for measuring near-atmospheric pressures of the solvents. In addition, the Pirani and ionization gauges are not operable at high temperatures, so both the Pirani and ionization gauges were moved from inside the thermal box to the line connecting the chamber and the turbomolecular pump, which is outside the insulated thermal box. These gauges were used to measure the extent of vacuum in the chamber.

A Leybold Turbovac 50 Turbomolecular pump was installed for the creation of high vacuum. The turbomolecular pump can reach a vacuum from $10^{-2}$ torr to $10^{-7}$ torr. The rotary vane pump is used as the roughing pump to reach vacuum in the range of $10^{-2}$ torr. The turbomolecular pump was installed outside the chamber and connected to the chamber via an elbow. The vent of the turbomolecular pump was also connected to the liquid nitrogen trap to prevent any contamination of the pump lubricant. A gate value was connected to the turbomolecular pump and a right angle valve was connected to the rotary vane pump through the liquid nitrogen trap. The gate valve was required to 
connect the vacuum chamber to the rotary vane pump or to the turbomolecular pump. This arrangement was necessary so that the vapors would not enter the turbomolecular pump while roughing the chamber using rotary vane pump. A cold water circulation line was connected to the turbomolecular pump to prevent any overheating of the pump.

Several more resistance temperature devices were installed in the thermal box and surface mounted onto the six-way cross to check for any temperature gradients in the thermal box and in the chamber.

The arrangement of the circulation fan and the heating element in the original QCM design caused the motor of the circulating fan to stop due to overheating. This was due to the high operating temperature of the thermal box. Therefore, the motor of the circulating fan was moved outside the thermal box to prevent any overheating of the motor. The motor was connected to the circulating fan inside the thermal box by a new aluminum shaft. The motor and the fan were vertically mounted onto one of the sides of thermal box to allow for better circulation of hot air in the thermal box. The heating element was mounted onto a metallic stand.

The original QCM design had a glass solvent flask. The line connecting the solvent flask was made of stainless tubing. Obtaining a leak-proof connection between the glass flask and the stainless steel tubing was extremely difficult. This affected the results of the QCM considerably, so the glass solvent flask was replaced by a jacketed metallic flask. Swagelok fittings were used to prevent any leaks from the flask. A new quick connect coupling was installed in the tubing connecting the solvent flask and the chamber to make the solvent change in the flask easy. A new magnetic stirring system was installed to 
provide good mixing of the solvent in the flask. The new solvent flask was also kept in a water bath heated by a constant circulation immersion heater to accurately maintain the temperature of the penetrant. The computer program recording the data from the deposition monitor was modified to record the data at smaller time intervals. 


\section{CHAPTER IV}

\section{RESULTS AND DISCUSSION}

Quartz crystal microbalance experiments were performed on the poly(vinyl acetate)-toluene system. The poly(vinyl acetate)-toluene system was selected in order to compare results of the QCM with experimental results of other researchers (Ju, 1981 and Hou, 1986) and the predicted values from the Free Volume theory (Zelinski and Duda, 1992). The poly(vinyl acetate)-toluene system is one of the few well characterized polymer-solvent systems with mutual diffusion data available in the literature. Therefore, poly(vinyl acetate)-toluene is an excellent candidate system to validate the accuracy of the QCM technique and the Free Volume theory.

Experiments were also conducted with two copolymer coatings from Rexham Graphics, Inc. The samples from Rexham Graphics were referred to as the 8883 and 8899 samples. Both the samples were mixtures of various copolymers, optical brighteners, polymeric resins, solvents, some low molecular weight additives and fillers such as $\mathrm{CaCO}_{3}$. Both the samples were used by Rexham Graphics as coatings on high value-added papers (e.g., Inkjet printer paper). The results of this work were used by Rexham Graphics in improving the drying characteristics of the polymer coatings studied.

For the QCM to provide accurate measurements, the frequency of the quartz crystals must be stable over a wide temperature range and should not exhibit temperature hysterisis. Experiments were performed with bare quartz crystals and with poly(vinyl 
acetate) coated quartz crystals for several temperature cycles to determine the extent of temperature hysterisis. The temperature dependence of bare and coated crystals are given in Figure 6. As is clear from Figure 6, the frequencies of bare and coated crystals are stable, reproducible, linear, and do not exhibit temperature hysterisis over the temperature range $40{ }^{\circ} \mathrm{C}$ to $80{ }^{\circ} \mathrm{C}$. Therefore, experiments can be performed with the same crystal at various temperatures within the range $40{ }^{\circ} \mathrm{C}-80{ }^{\circ} \mathrm{C}$, without measuring the bare crystal frequencies at different temperatures.

\section{A. POLY(VINYL ACETATE)-TOLUENE SYSTEM}

Experiments were performed with the poly(vinyl acetate)-toluene system at polymer temperatures of $40^{\circ} \mathrm{C}, 60^{\circ} \mathrm{C}$, and $80{ }^{\circ} \mathrm{C}$. Experiments were performed at these temperatures by maintaining the thermal box around the vacuum chamber at the required temperature with a heater as described in Chapter III. Several runs were performed with poly(vinyl acetate)-toluene system at each of the three temperatures. The results of the diffusivity experiments were also compared with the predictions of Free Volume theory of Zelinski and Duda (1992) and also with the experimental results of other researchers (Ju, 1981 and Hou, 1986) using a quartz spring balance.

Knowledge of equilibrium conditions is necessary to specify the boundary conditions in the modeling of industrial applications (e.g., drying of polymer coatings). Activity or activity coefficient data are required for the solubility predictions in polymersolvent systems and also to study the phase equilibria thermodynamics of polymeric 


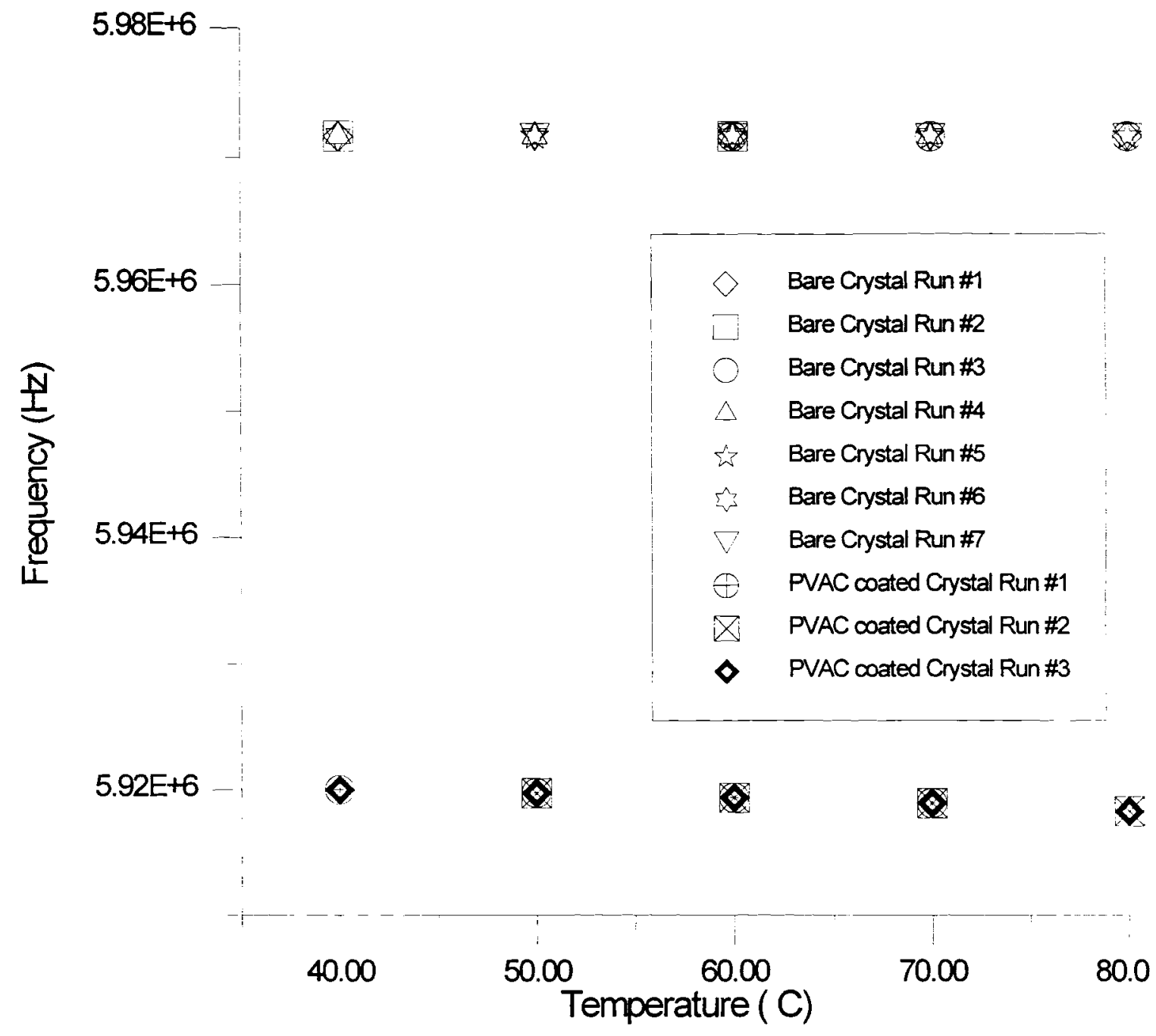

Figure 6.

Temperature Dependence of the Frequency of the Bare and Coated Quartz Crystals 
systems. Therefore, activities and activity coefficients were also measured via the QCM. The results of these experiments are shown in Table II.

The diffusivity and equilibrium sorption results of the QCM experiments, the calculated values of the diffusion coefficients from Free Volume theory, equilibrium sorption predictions from thermodynamic models and the experimental results of other researchers using a quartz spring balance are presented in Figures 7-12. As evident from Figure 7 , the diffusion coefficients at $40{ }^{\circ} \mathrm{C}$ agree (within error limits of $\pm 20 \%$ ) with the quartz spring data of $\mathrm{Ju}(1981)$ and with the predictions of Free Volume theory. Sorption experiments at $40{ }^{\circ} \mathrm{C}$ are very difficult and error prone because sorption takes place very slowly and the QCM has a small response. However, the QCM produces larger responses than other gravimetric techniques at lower temperatures.

Each of the runs was performed with multiple, small step changes in the pressure of the solvent in the chamber. The results of the experiments were analyzed with the assumption of a constant diffusivity. The effect of the constant diffusion coefficient assumption was minimized by making the diffusion driving force as small as possible. This was achieved by introducing small amounts of penetrant into the chamber. The chamber pressure was used to calculate the activity and the activity coefficient of the solvent. After equilibrium was reached for a given sorption run, the next sorption run was conducted by introducing an additional, small amount of penetrant into the chamber and the weight uptake was recorded. The penetrant was introduced into the chamber until the pressure was close to, but not greater than, the vapor pressure of the solvent at the 


\section{Table II}

Equilibrium Sorption and Diffusivity of Toluene and Poly(vinyl acetate) Measured via the Quartz Crystal Microbalance

\begin{tabular}{|c|c|c|c|c|c|}
\hline $\begin{array}{l}\text { Temp } \\
\left({ }^{\circ} \mathrm{C}\right)\end{array}$ & $\begin{array}{l}\text { Run } \\
\text { No. }\end{array}$ & $\begin{array}{l}\text { Equilibrium } \\
\text { Solvent Conc. } \\
\text { (wt \%) }\end{array}$ & Activity & $\begin{array}{l}\text { Average } \\
\text { Solvent Conc. } \\
\text { (wt \%) }\end{array}$ & $\begin{array}{l}\text { Diffusivity } \\
\left(\mathrm{cm}^{2} / \mathrm{sec}\right)\end{array}$ \\
\hline \multirow[t]{3}{*}{40} & 1 & 8.83 & 0.363 & 6.18 & $7.85 \times 10^{-11}$ \\
\hline & $2 a$ & 4.49 & 0.227 & 3.15 & $2.70 \times 10^{-11}$ \\
\hline & $2 b$ & 11.2 & 0.494 & 9.25 & $2.97 \times 10^{-9}$ \\
\hline \multirow[t]{25}{*}{60} & $1 \mathrm{a}$ & 1.30 & 0.096 & 0.91 & $1.64 \times 10^{-10}$ \\
\hline & $1 b$ & 3.21 & 0.204 & 2.64 & $4.68 \times 10^{-10}$ \\
\hline & $1 \mathrm{c}$ & 5.87 & 0.316 & 5.08 & $1.62 \times 10^{-9}$ \\
\hline & $1 d$ & 7.54 & 0.376 & 6.80 & $4.47 \times 10^{-9}$ \\
\hline & le & 10.53 & 0.485 & 9.63 & $3.72 \times 10^{-8}$ \\
\hline & $2 a$ & 1.95 & 0.129 & 1.36 & $2.20 \times 10^{-10}$ \\
\hline & $2 b$ & 4.90 & 0.264 & 4.02 & $9.22 \times 10^{-10}$ \\
\hline & $2 c$ & 8.56 & 0.393 & 7.46 & $5.19 \times 10^{-9}$ \\
\hline & $2 d$ & 11.87 & 0.484 & 10.88 & $3.68 \times 10^{-8}$ \\
\hline & $3 a$ & 1.66 & 0.113 & 1.16 & $2.00 \times 10^{-10}$ \\
\hline & $3 b$ & 3.88 & 0.224 & 3.21 & $5.49 \times 10^{-10}$ \\
\hline & $3 c$ & 6.77 & 0.336 & 6.77 & $2.14 \times 10^{-9}$ \\
\hline & $3 d$ & 10.54 & 0.448 & 9.41 & $1.30 \times 10^{-8}$ \\
\hline & $3 e$ & 15.23 & 0.558 & 13.82 & $7.19 \times 10^{-8}$ \\
\hline & $4 a$ & 2.06 & 0.112 & 1.44 & $4.29 \times 10^{-10}$ \\
\hline & $4 b$ & 4.53 & 0.227 & 3.79 & $1.45 \times 10^{-9}$ \\
\hline & $5 a$ & 1.97 & 0.114 & 1.38 & $3.49 \times 10^{-10}$ \\
\hline & $5 b$ & 4.34 & 0.228 & 3.63 & $8.77 \times 10^{-10}$ \\
\hline & $5 c$ & 7.48 & 0.342 & 6.54 & $3.16 \times 10^{-9}$ \\
\hline & $6 a$ & 4.04 & 0.167 & 2.83 & $1.06 \times 10^{-9}$ \\
\hline & $6 b$ & 9.35 & 0.369 & 7.95 & $3.17 \times 10^{-8}$ \\
\hline & $6 c$ & 7.72 & 0.334 & 5.41 & $1.70 \times 10^{-9}$ \\
\hline & $7 \mathrm{a}$ & 5.43 & 0.054 & 3.80 & $9.94 \times 10^{-10}$ \\
\hline & $7 b$ & 10.93 & 0.109 & 9.28 & $2.42 \times 10^{-8}$ \\
\hline & $7 \mathrm{c}$ & 20.40 & 0.204 & 17.56 & $5.63 \times 10^{-8}$ \\
\hline \multirow[t]{5}{*}{80} & $1 \mathrm{a}$ & 2.02 & 0.097 & 1.42 & $3.15 \times 10^{-8}$ \\
\hline & $1 \mathrm{~b}$ & 4.34 & 0.180 & 3.64 & $3.65 \times 10^{-8}$ \\
\hline & $1 \mathrm{c}$ & 6.50 & 0.286 & 5.86 & $7.48 \times 10^{-8}$ \\
\hline & $1 d$ & 9.65 & 0.388 & 8.71 & $1.63 \times 10^{-7}$ \\
\hline & le & 14.50 & 0.531 & 13.05 & $1.79 \times 10^{-7}$ \\
\hline
\end{tabular}




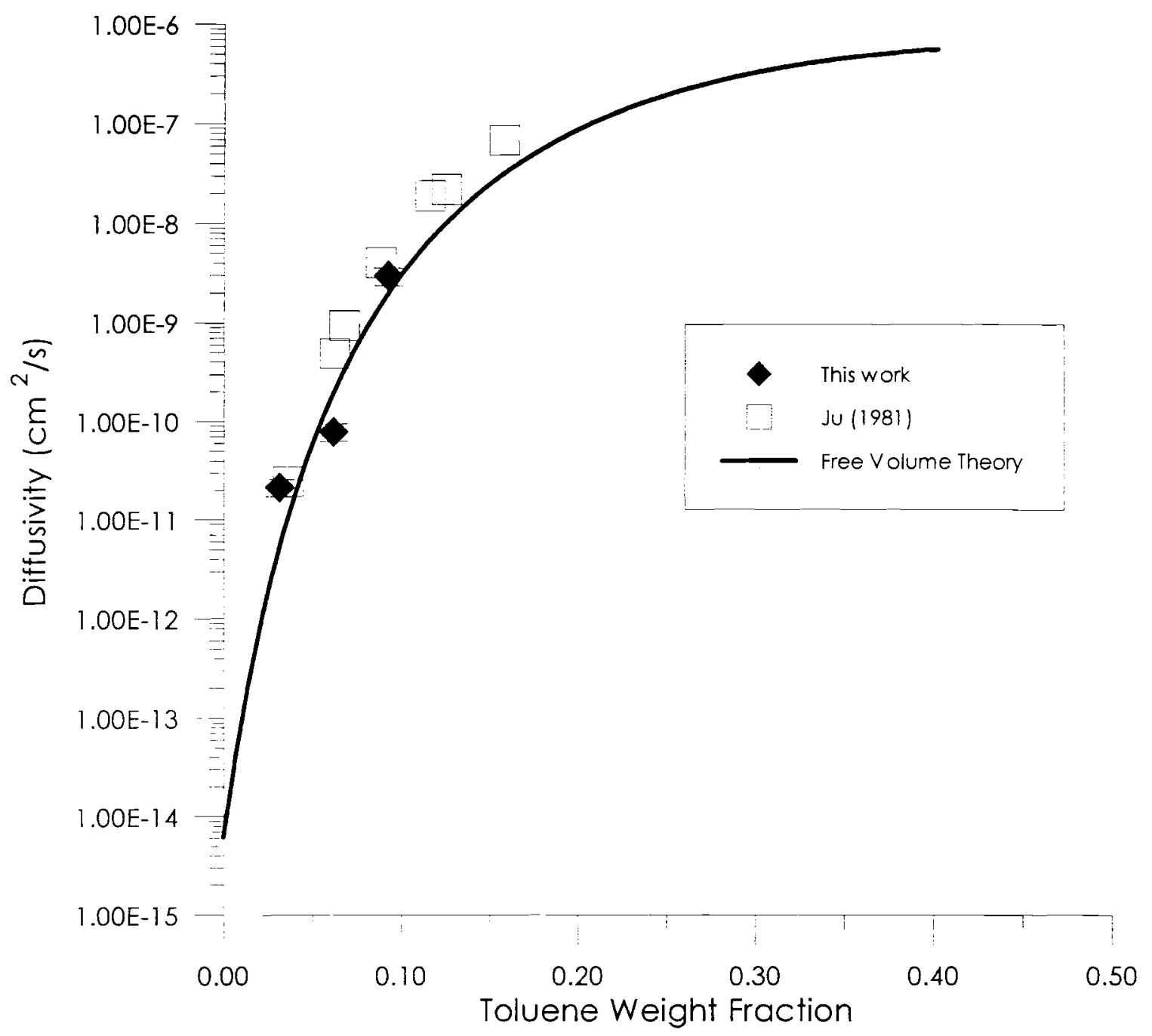

Figure 7.

Mutual Diffusivity of Toluene and Poly(vinyl acetate) at $40{ }^{\circ} \mathrm{C}$ for the Quartz Spring Balance (Ju, 1981), Quartz Crystal Microbalance, and the Free Volume Theory 


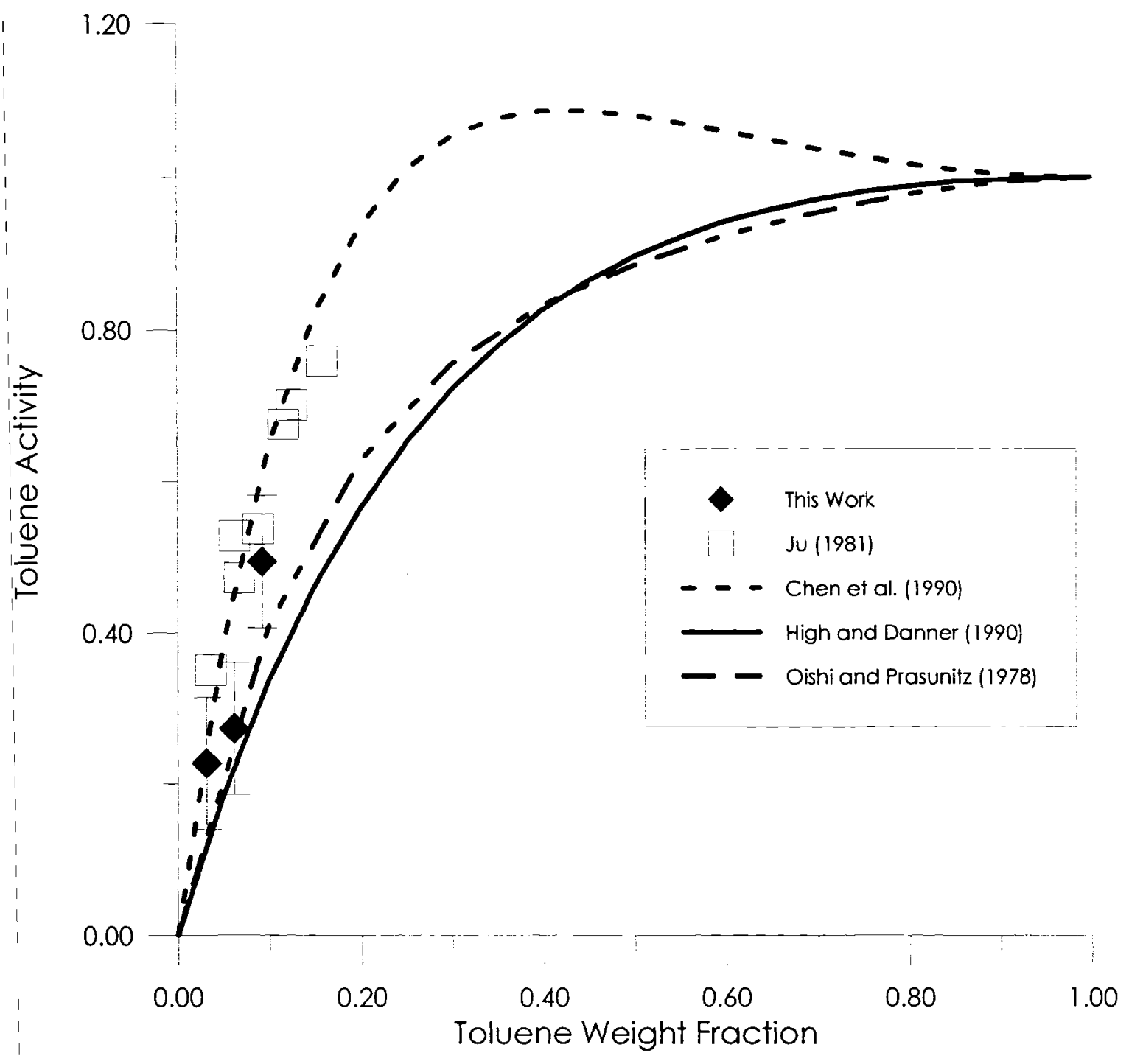

Figure 8.

Equilibrium Sorption Experiments of Toluene and Poly(vinyl acetate) at $40{ }^{\circ} \mathrm{C}$ 


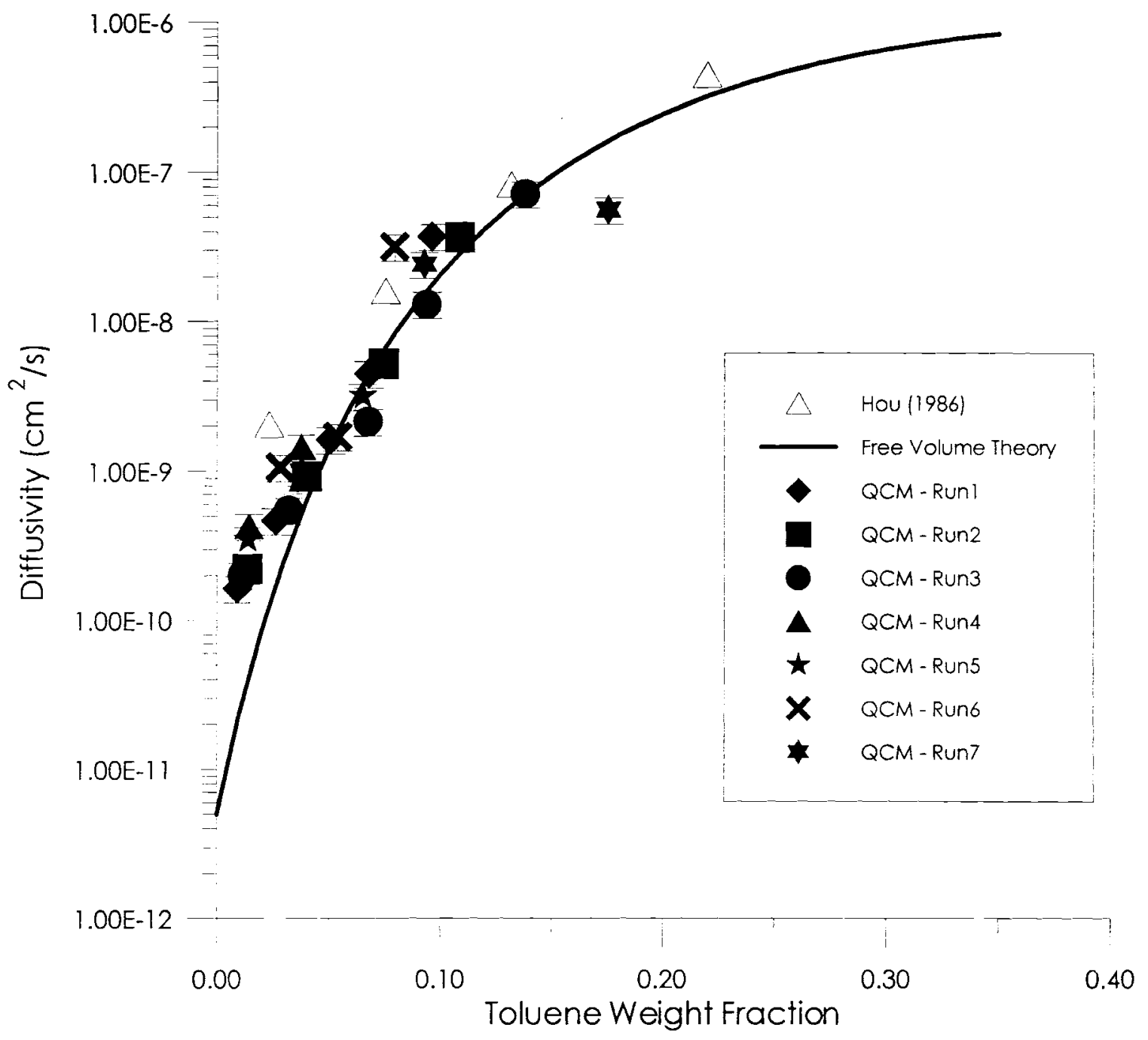

Figure 9.

Mutual Diffusivity of Toluene and Poly(vinyl acetate) at $60{ }^{\circ} \mathrm{C}$ for the Quartz Spring Balance (Hou, 1986), Quartz Crystal Microbalance, and the Free Volume Theory 


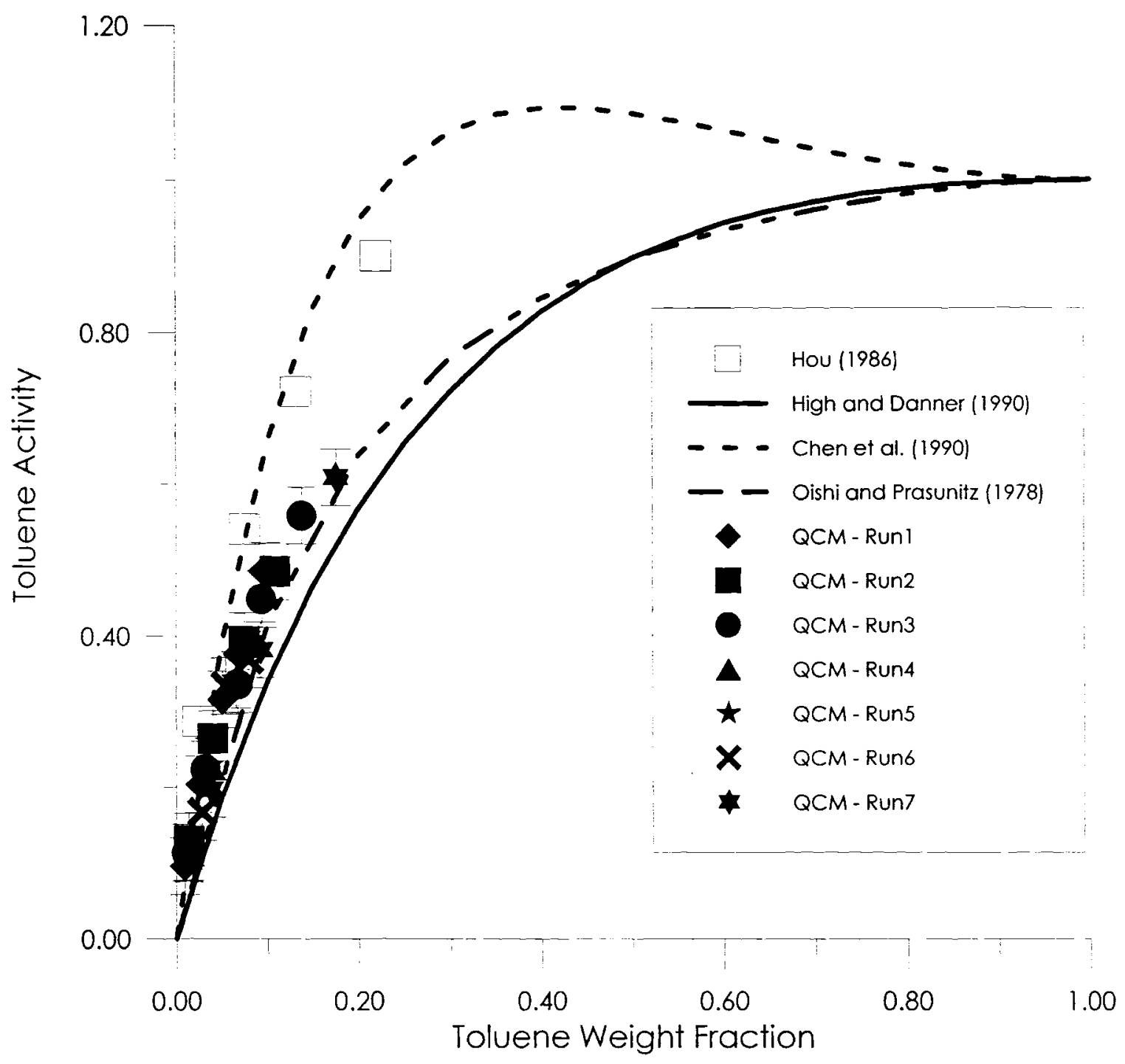

Figure 10.

Equilibrium Sorption Experiments of Toluene and Poly(vinyl acetate) at $60{ }^{\circ} \mathrm{C}$ 


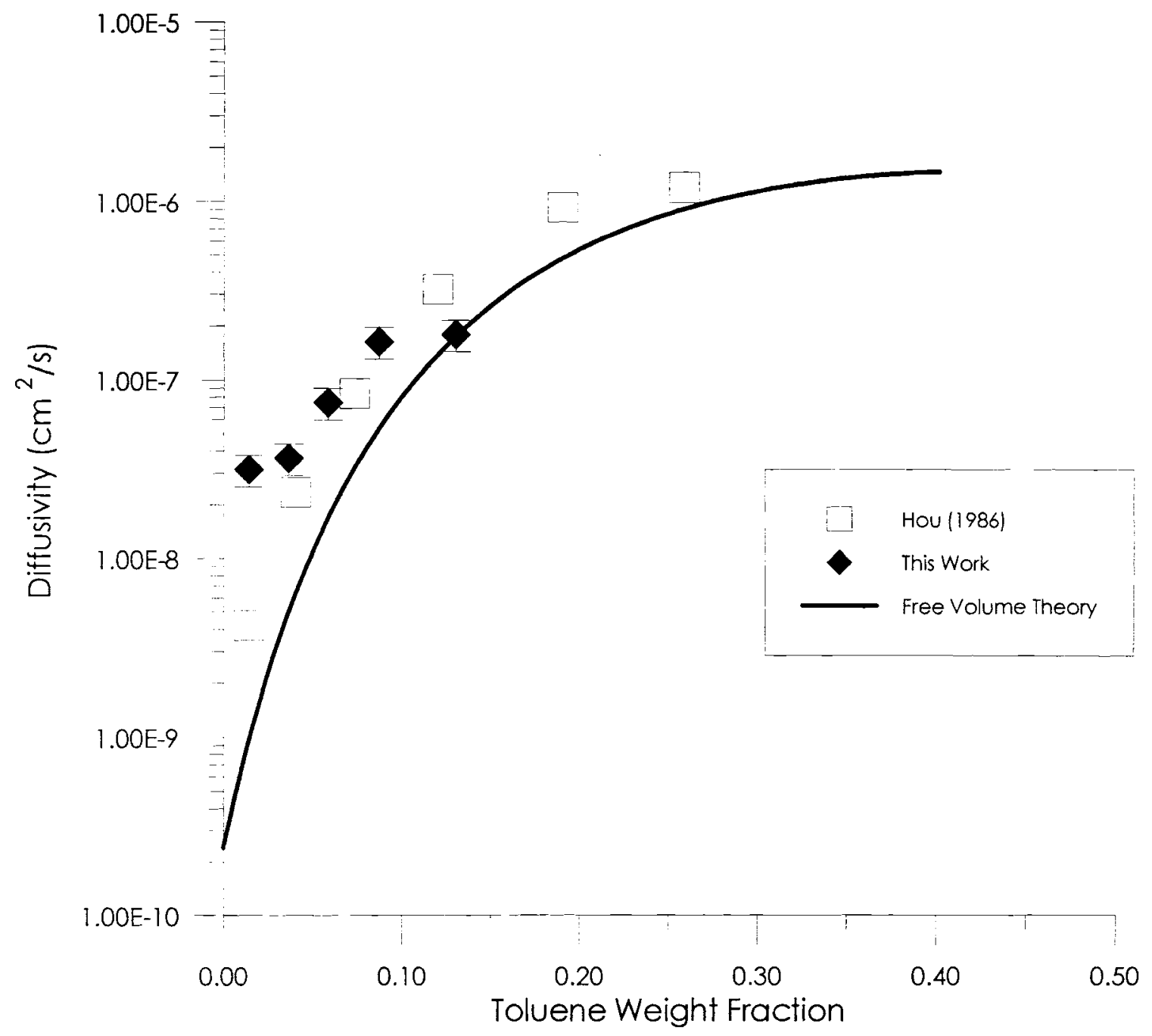

Figure 11.

Mutual Diffusivity of Toluene and Poly(vinyl acetate) at $80{ }^{\circ} \mathrm{C}$ for the Quartz Spring Balance (Hou, 1986), Quartz Crystal Microbalance, and the Free Volume Theory 


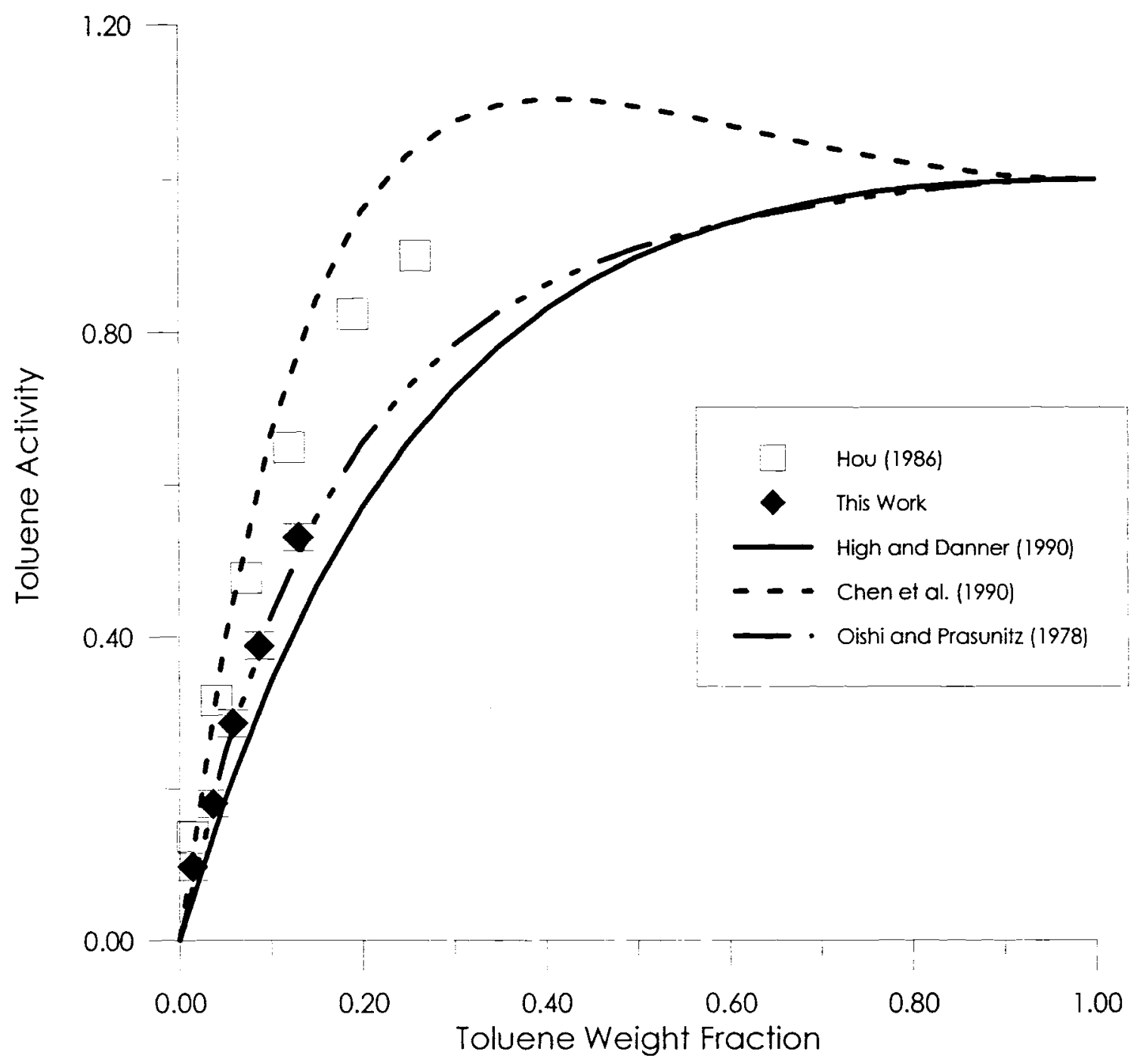

Figure 12.

Equilibrium Sorption Experiments of Toluene and Poly(vinyl acetate) at $80{ }^{\circ} \mathrm{C}$ 
chamber temperature. An ideal step change of pressure was assumed for data analysis each time penetrant was introduced into the chamber.

As can be seen from Figures 9 and 11 the diffusion coefficients from the quartz crystal microbalance at $60{ }^{\circ} \mathrm{C}$ and $80{ }^{\circ} \mathrm{C}$ are in agreement (within error limits of $\pm 20 \%$ ) with the diffusion coefficients obtained by Hou (1986). The diffusions coefficients at 40 ${ }^{\circ} \mathrm{C}, 60{ }^{\circ} \mathrm{C}$ and $80{ }^{\circ} \mathrm{C}$ (Figures 7, 9, and 11) agree (within error limits of $\pm 20 \%$ ) with the values calculated from the Free Volume theory. Additional results are needed at $40{ }^{\circ} \mathrm{C}$ to further quantify the accuracy of results of the QCM at $40{ }^{\circ} \mathrm{C}$.

Experimental results for the equilibrium solubility of PVAC-toluene at $40{ }^{\circ} \mathrm{C}$, $60{ }^{\circ} \mathrm{C}$, and $80^{\circ} \mathrm{C}$ are given in Figures 8,10 , and 12 , along with the predicted values from the models of Oishi and Prasunitz (1978), Chen et al. (1990) and High and Danner (1990). These models were chosen because they are the only models available that have been shown to be capable of predicting polymer-solvent thermodynamic properties. Although only three data points are available, the $40{ }^{\circ} \mathrm{C}$ QCM values are in agreement (within error limits of $\pm 20 \%$ ) with the Oishi and Prasunitz model, and the quartz spring data of Ju (1981). The equilibrium sorption results of the QCM at $60{ }^{\circ} \mathrm{C}$ and $80{ }^{\circ} \mathrm{C}$ clearly do not agree with the quartz spring data (Hou, 1986), but are in good agreement with the Oishi-Prasunitz model. The High-Danner model slightly underpredicts the toluene activities at all three temperatures.

The equilibrium sorption results of the QCM are lower than those of quartz spring data at all the temperatures at which the experiments were performed. This consistent difference is most likely due to the use of different poly(vinyl acetate) resin. The effect 
of different resins can only be confirmed by performing the experiments on QCM and the quartz spring balance with the same poly(vinyl acetate) resin. Poly(vinyl acetate) samples have been sent to another laboratory for quartz spring measurements.

At all the three temperatures $40{ }^{\circ} \mathrm{C}, 60^{\circ} \mathrm{C}$, and $80{ }^{\circ} \mathrm{C}$, the Chen et al. (1990) model significantly overpredicts both the quartz spring data and the QCM data. In addition, the Chen et al. (1990) model predicts that the activity values exceed unity, which indicates that the toluene-poly(vinyl acetate) system phase separates. Qualitative experiments indicate that toluene-poly(vinyl acetate) system is miscible at all concentrations in the temperature range of $40{ }^{\circ} \mathrm{C}$ to $80^{\circ} \mathrm{C}$. Known weights of poly(vinyl acetate) and toluene were mixed in a bottle, and the bottle was heated by inserting it into the thermal box, which was already at the required temperature. This qualitative experiments were done at three temperatures $40{ }^{\circ} \mathrm{C}, 60^{\circ} \mathrm{C}$, and $80{ }^{\circ} \mathrm{C}$. The experimental results indicate that toluene-poly(vinyl acetate) system is miscible at all the concentrations and at the three temperatures of interest. Therefore, the results of these qualitative experiments indicate that the predictions of the Chen et al. model are erroneous.

A number of results lead to the conclusion that the QCM data obtained in this study are the more accurate than those of Hou (1986) and Ju (1981). First, the high reproducibility of the QCM data as demonstrated in Figure 9 indicates that the technique is not affected by changes in the crystal or polymer coating. The different QCM runs in Figure 9 were obtained from different quartz crystals and different polymer coatings resulting in a range of film thicknesses. Secondly, the agreement among the QCM data 
and Oishi-Prasunitz model, rather than the Chen et al. model, weigh in favor of the QCM data over the quartz spring data of Hou (1986) and Ju (1981). The Oishi-Prasunitz model has been shown to be superior to the Chen et al. model in most cases (High and Danner, 1990). In addition, the Chen model indicates that the toluene-poly(vinyl acetate) system phase separates at approximately 50 weight percent, which is contrary to the qualitative experiments discussed above. Since the quartz spring data of Ju (1981) and Hou (1986) follow the trend of the Chen et al. model at low concentrations, their data appear to be in error. Since the QCM data are reproducible and more clearly match the results of more accurate, well-behaved model, the conclusion can be reached that the QCM data are more accurate. The experiments performed with other gravimetric sorption techniques such as quartz spring balance take a long time, typically about two weeks per run (multiple step changes). However, the experiments on a QCM can be performed in a short time interval, typically about a day per run (multiple step changes). Therefore, experiments can be performed faster on a QCM than on any other technique which is based on gravimetric sorption.

\section{B. INDUSTRIAL SAMPLES: CASE STUDY I}

The ultimate goal of the QCM is to provide solubility and diffusivity data for industrially relevant systems. In this study, the diffusion characteristics of toluene in the 8883 and 8899 samples from Rexham Graphics Inc. were studied using the QCM. A known volume of both the 8883 and 8899 samples were dried in a vacuum oven to determine the weight percentage of solids in the samples, which were found to be $66 \%$ 
and $55 \%$, respectively. The samples were coated onto quartz crystals after diluting in tetrahydrofuran. QCM sorption experiments were performed with the coated crystals to study the diffusion and equilibrium sorption characteristics of toluene in both 8883 and 8899 samples. Several experiments were performed at film temperatures of $60{ }^{\circ} \mathrm{C}$ and $80^{\circ} \mathrm{C}$.

Equilibrium weight fractions and diffusivities of toluene in the 8883 and 8899 samples at various concentrations were calculated from the sorption experiments performed. The results of the experiments are shown in Table III and Table IV. The concentrations corresponding to the diffusivities in the tables are the average solvent concentrations. The diffusivity of toluene in 8883 at a film temperature of $60{ }^{\circ} \mathrm{C}$ increases in the lower concentration range and remains almost constant at higher concentrations as can be observed from Figure 13. This behavior of 8883-toluene system is similar to the diffusion characteristics of poly(vinyl acetate)-toluene system. Figure 14 indicates that at a film temperature of $80^{\circ} \mathrm{C}$, the diffusivity of toluene in 8883 sample increases in the lower concentration range and then decreases slightly at higher concentrations. The results in Table III, Figure 13 and Figure 14 indicate that the mutual diffusivity of the toluene- 8883 sample is not a strong function of temperature or concentration. Figures 15 and 16 indicate that the diffusivities of toluene in 8899 samples reach a maximum and then drops with the increase in concentration. Figure 15 indicates that the diffusivity of toluene in 8899 sample at a film temperature of $60{ }^{\circ} \mathrm{C}$ is a strong function of concentration and varies by an order of magnitude. The diffusion characteristics of 8899-toluene system is similar to the diffusion characteristics of 


\section{Table III}

Equilibrium Sorption and Diffusivity of Toluene in 8883 Sample Measured via the Quartz Crystal Microbalance

Equilibrium

Temp Run No. Solvent $\left({ }^{\circ} \mathrm{C}\right)$

Conc.

(wt \%)

$\begin{array}{ll}1 \mathrm{a} & 1.0 \\ 1 \mathrm{~b} & 2.1 \\ 1 \mathrm{c} & 3.7 \\ 1 \mathrm{~d} & 6.4 \\ 2 \mathrm{a} & 1.0 \\ 2 \mathrm{~b} & 2.0 \\ 2 \mathrm{c} & 4.0 \\ 2 \mathrm{~d} & 6.3 \\ 3 \mathrm{a} & 1.0 \\ 3 \mathrm{~b} & 2.3 \\ 3 \mathrm{c} & 3.8 \\ 4 \mathrm{a} & 1.0 \\ 4 \mathrm{~b} & 2.5 \\ 4 \mathrm{c} & 3.7 \\ 4 \mathrm{~d} & 5.7\end{array}$

1.0

0.189

17.8

$0.523 \quad 13.9$

$0.683 \quad 10.5$

0.338

17.8

0.552

13.5

0.686

0.189

0.373

0.528

0.190

0.406

0.524

0.634

80

$\begin{array}{ll}1 \mathrm{a} & 0.7 \\ 1 \mathrm{~b} & 1.4 \\ 1 \mathrm{c} & 2.5 \\ 1 \mathrm{~d} & 3.9 \\ 1 \mathrm{e} & 6.6 \\ \mathrm{2a} & 0.7 \\ \mathrm{2b} & 1.4 \\ \mathrm{2c} & 2.4 \\ 2 \mathrm{~d} & 4.1 \\ 2 \mathrm{e} & 6.1 \\ 3 \mathrm{a} & 0.7 \\ 3 \mathrm{~b} & 1.4 \\ 3 \mathrm{c} & 2.4 \\ 3 \mathrm{~d} & 4.0 \\ \mathrm{3e} & 6.0\end{array}$

0.131

18.5

17.7

0.397

15.8

0.527

13.5

0.687

10.3

0.127

18.2

0.251

17.5

0.376

15.9

0.536

13.1

0.658

10.7

0.130

18.3

0.255

17.5

0.380

15.9

0.533

13.3

0.669

11.1

0.7

1.8

3.2

5.7

0.7

1.7

3.5

5.7

0.7

1.9

3.4

0.7

2.1

3.3

5.1

0.5

1.2

2.2

3.5

5.8

0.5

1.2

2.1

3.5

5.5

$5.87 \times 10^{-9}$

$8.79 \times 10^{-9}$

$8.09 \times 10^{-9}$

$1.01 \times 10^{-8}$

$7.72 \times 10^{-9}$

$9.70 \times 10^{-9}$

$8.68 \times 10^{-9}$

$8.45 \times 10^{-9}$

$5.48 \times 10^{-9}$

$1.17 \times 10^{-8}$

$1.30 \times 10^{-8}$

$7.86 \times 10^{-9}$

$7.72 \times 10^{-9}$

$1.27 \times 10^{-8}$

$8.69 \times 10^{-9}$

$9.42 \times 10^{-9}$

$9.96 \times 10^{-9}$

$1.03 \times 10^{-8}$

$1.03 \times 10^{-8}$

$6.17 \times 10^{-9}$

$8.71 \times 10^{-9}$

$8.40 \times 10^{-9}$

$9.73 \times 10^{-9}$

$1.01 \times 10^{-8}$

$6.18 \times 10^{-9}$

$8.33 \times 10^{-9}$

1.2

$9.65 \times 10^{-9}$

2.1

3.5

$1.12 \times 10^{-8}$

$9.67 \times 10^{-9}$

5.4

$7.37 \times 10^{-9}$ 
Table IV

Equilibrium Sorption and Diffusivity of Toluene in 8899 sample Measured via the Quartz Crystal Microbalance

Equilibrium

Temp Run No. Solvent

$\left({ }^{\circ} \mathrm{C}\right)$

Conc.(wt \%)

60

$\begin{array}{ll}1 \mathrm{a} & 3.0 \\ 1 \mathrm{~b} & 7.4 \\ 1 \mathrm{c} & 16.3\end{array}$

$2 \mathrm{a}$

$2 \mathrm{~b}$

$2 \mathrm{c}$

$3 \mathrm{a}$

$3 b$

$3 c$

$3 \mathrm{~d}$

$3 \mathrm{e}$

$4 a$

$4 b$

$4 \mathrm{c}$

$4 d$

80
3.3

6.7

21.6

1.9

3.8

6.4

10.2

17.3

2.0

4.0

6.7

17.2

0.8

2.0

3.5

5.2

7.7

10.9

15.8

1.4

3.0

5.1

8.0

12.5

17.5

2.0

4.3

7.7

13.0

18.8
Activity Activity

Coefficien

0.189

0.376

0.559

0.190

0.341

0.598

0.115

0.227

0.340

0.457

0.570

0.114

0.229

0.341

0.559

0.093

0.180

0.270

0.357

0.450

0.538

0.630

0.096

0.198

0.312

0.421

0.533

0.622

0.129

0.251

0.376

0.500

0.621
6.3

5.1

3.4

5.8

5.0

2.8

6.1

5.9

5.3

4.5

3.3

5.7

5.7

5.0

3.2

11.0

8.8

7.7

6.8

5.8

4.9

3.9

7.0

6.6

6.0

5.2

4.2

3.5

6.3

5.8

4.9

3.8

3.3
2.1

6.0

13.6

2.3

5.7

17.1

1.3

3.2

5.6

9.0

15.1

1.4

3.5

5.9

14.0

0.6

1.7

3.1

4.7

7.0

9.9

14.3

0.9

2.5

4.5

7.2

11.2

16.0

1.4

3.6

6.6

11.4

17.0
$1.04 \times 10^{-8}$

$2.27 \times 10^{-8}$

$3.07 \times 10^{-8}$

$1.89 \times 10^{-8}$

$2.97 \times 10^{-8}$

$2.18 \times 10^{-8}$

$5.98 \times 10^{-9}$

$2.10 \times 10^{-8}$

$3.87 \times 10^{-8}$

$4.13 \times 10^{-8}$

$3.57 \times 10^{-8}$

$7.58 \times 10^{-9}$

$2.46 \times 10^{-8}$

$2.93 \times 10^{-8}$

$4.97 \times 10^{-8}$

$3.27 \times 10^{-8}$

$2.68 \times 10^{-8}$

$4.44 \times 10^{-8}$

$5.10 \times 10^{-8}$

$5.41 \times 10^{-8}$

$4.31 \times 10^{-8}$

$2.04 \times 10^{-8}$

$2.52 \times 10^{-8}$

$3.09 \times 10^{-8}$

$5.14 \times 10^{-8}$

$5.08 \times 10^{-8}$

$4.83 \times 10^{-8}$

$2.32 \times 10^{-8}$

$2.44 \times 10^{-8}$

$2.94 \times 10^{-8}$

$4.87 \times 10^{-8}$

$3.77 \times 10^{-8}$

$2.14 \times 10^{-8}$ 


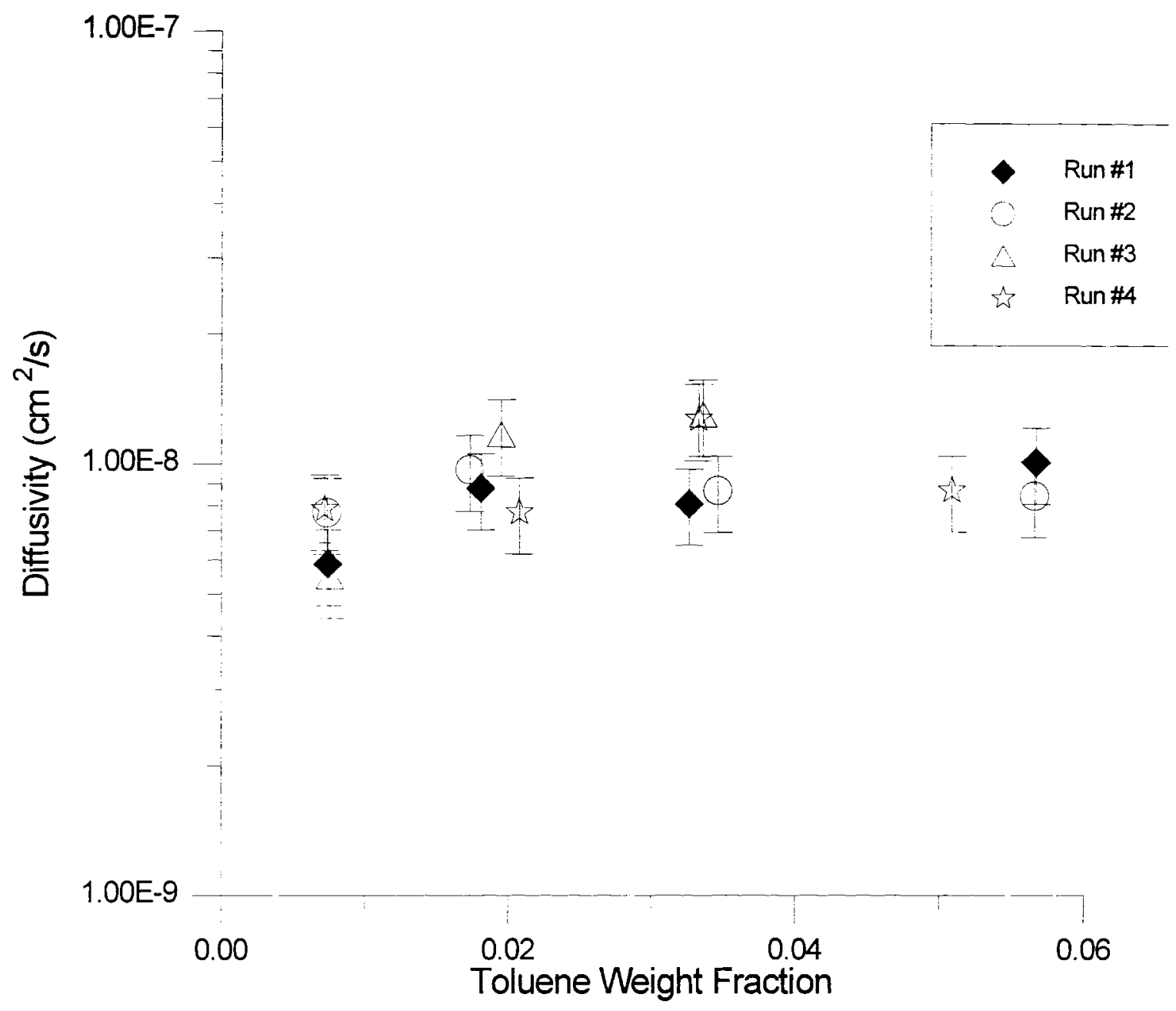

Figure 13.

Mutual Diffusivity of Toluene and 8883 Sample at $60{ }^{\circ} \mathrm{C}$ for the Quartz Crystal Microbalance 


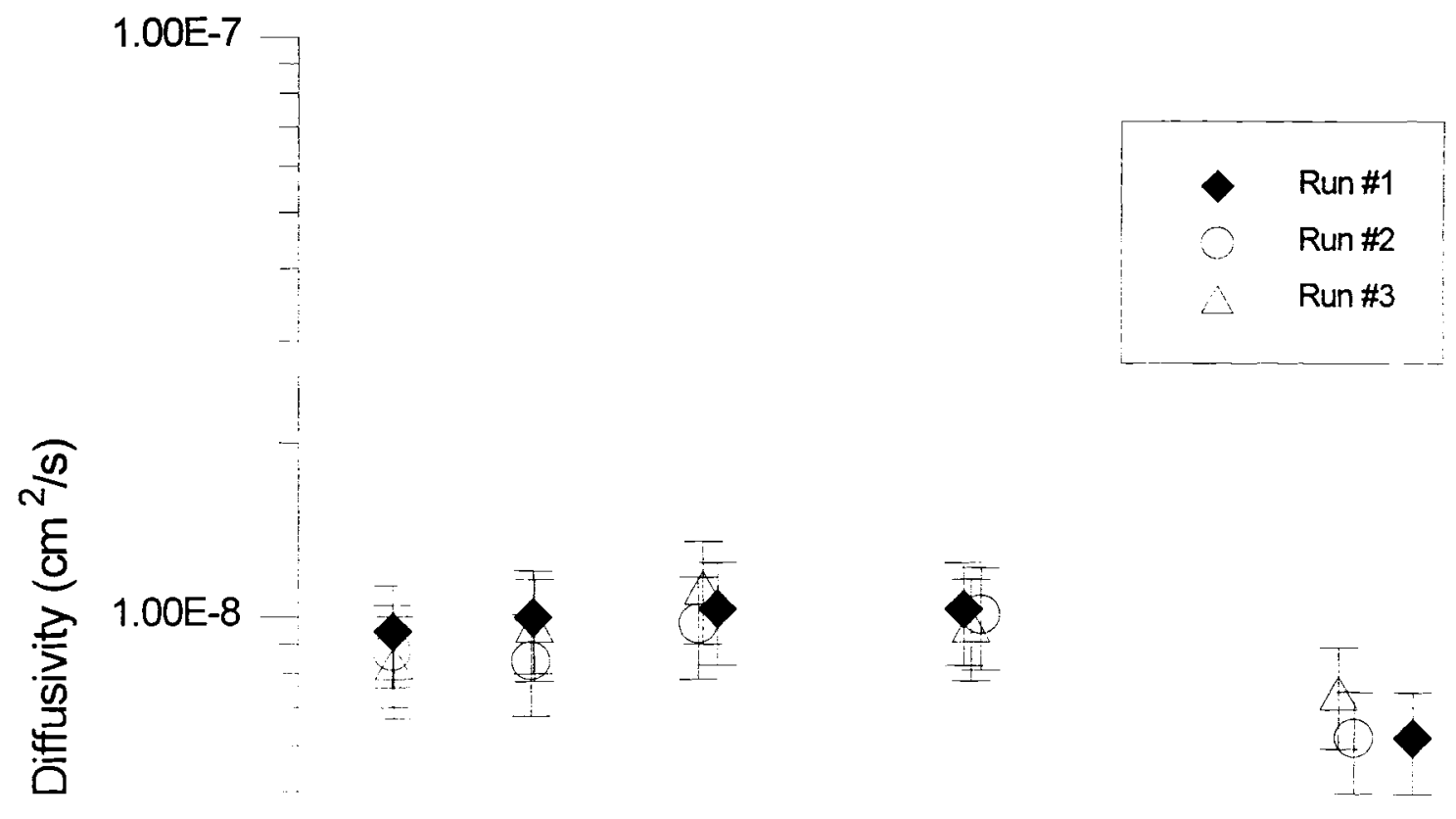

$1.00 \mathrm{E}-9$

0.00

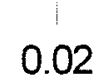

Toluene Weight Fraction

Figure 14.

Mutual Diffusivity of Toluene and 8883 Sample at $80^{\circ} \mathrm{C}$ for the Quartz Crystal

Microbalance 


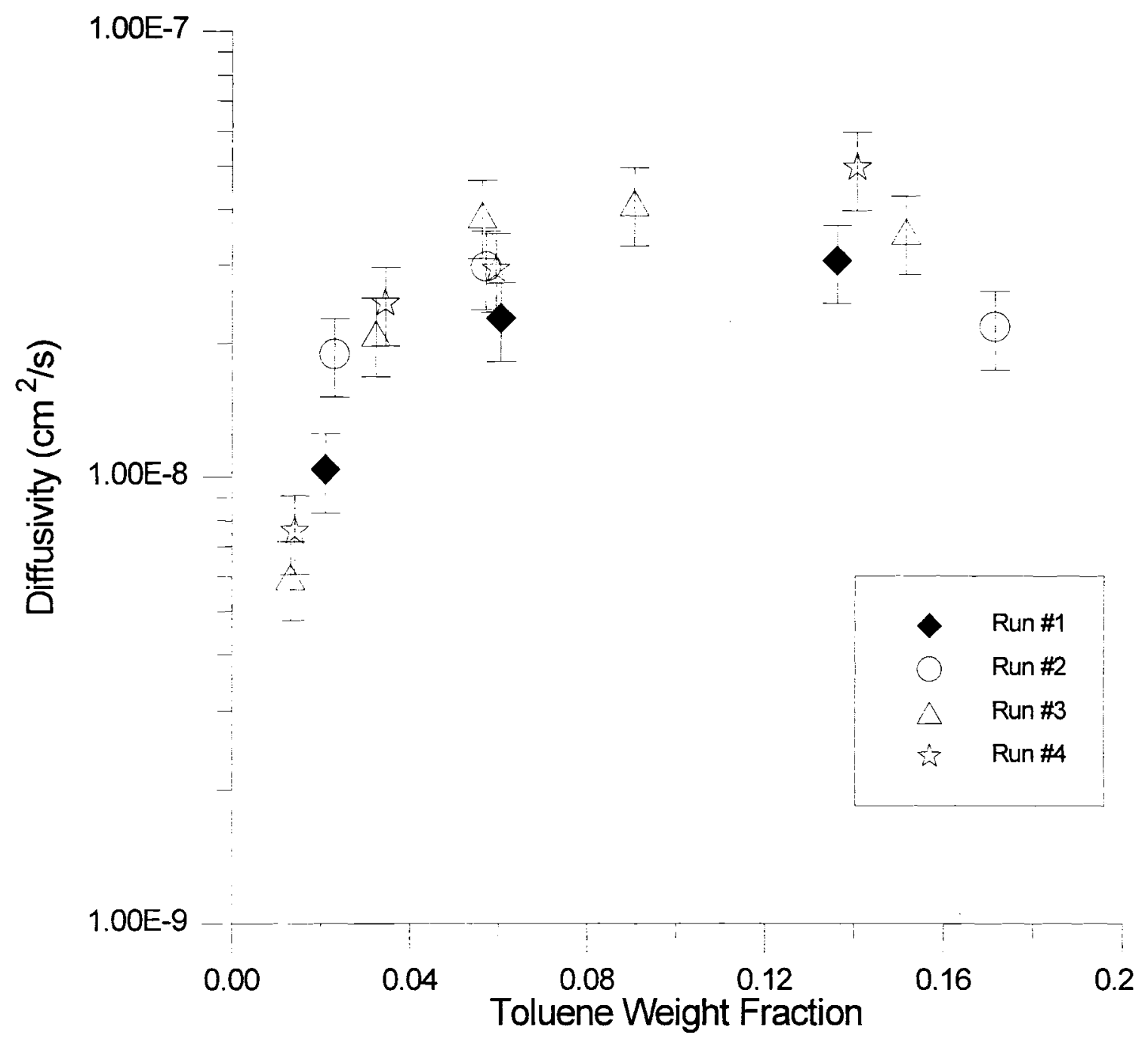

Figure 15.

Mutual Diffusivity of Toluene and 8899 Sample at $60^{\circ} \mathrm{C}$ for the Quartz Crystal Microbalance 


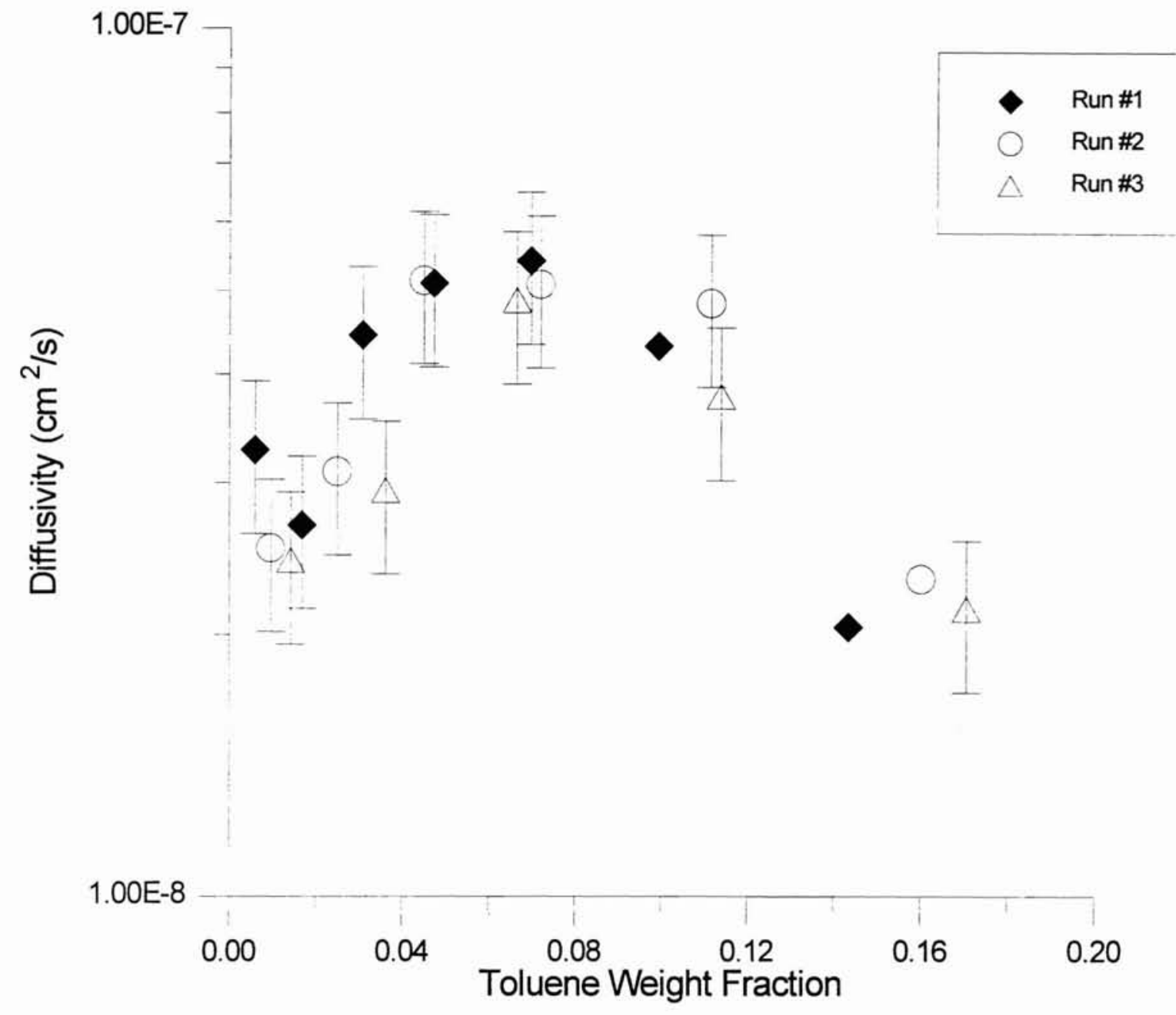

Figure 16.

Mutual Diffusivity of Toluene and 8899 Sample at $80{ }^{\circ} \mathrm{C}$ for the Quartz Crystal

Microbalance 
toluene-polystyrene system (Zelinski and Duda, 1992) in that diffusivity first increases then decreases with concentration.

The exact nature of diffusion of toluene into the 8883 and the 8899 samples is not clearly understood. Both the 8883 and the 8899 samples are filled polymers mixed with several optical brighteners and low molecular weight additives making the diffusion phenomena very complex. Little attention has been devoted in studying the diffusion characteristics of solvents in filled polymers. More systematic studies need to be done to clearly understand the complex diffusion characteristics of solvents in filled polymeric systems.

Comparison of Tables III and IV indicate that the equilibrium weight fraction of toluene in 8899 sample is much greater than the weight fraction of toluene in 8883 sample. Figures 17, 18, 19, and 20, indicate the activity coefficient of toluene is much greater in 8883 sample than in 8899 sample. As can be seen from Figures 17, 18, and 19, the equilibrium sorption results of the 8883 and 8899 samples were reproducible. But, from Figure 20 it can be seen that, the equilibrium sorption results at $80{ }^{\circ} \mathrm{C}$ were not as reproducible. The sorption results of run $\# 1$ were higher than those of run $\# 2$ and run $\# 3$. More experiments should be performed with the 8899 sample to check the validity of run \#1 results.

\section{INDUSTRIAL SAMPLES: CASE STUDY II}

Experiments were also performed with an industrial copolymer sample from Dow Chemical Company to check for the equilibrium sorption characteristics of the 


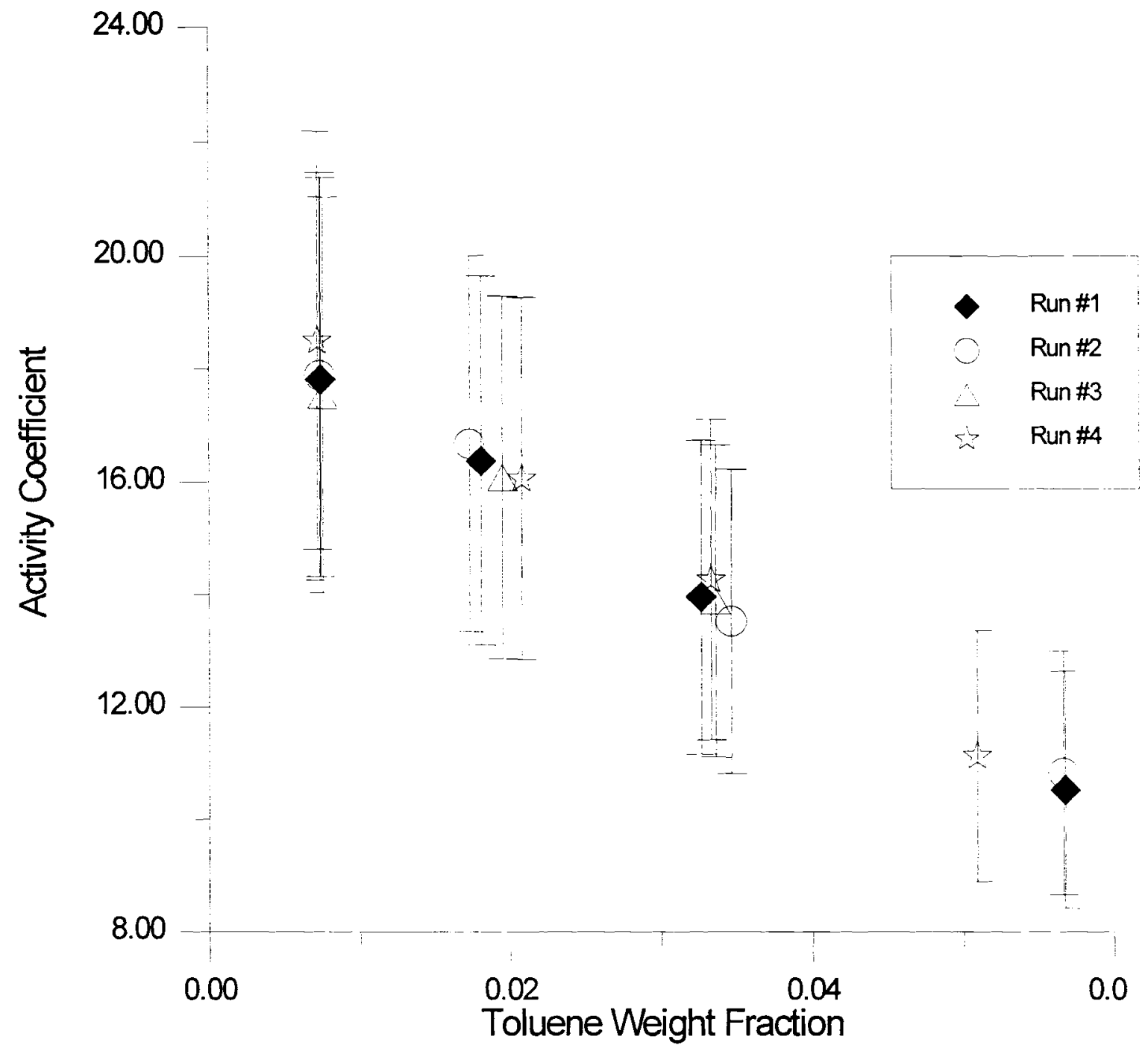

Figure 17.

Equilibrium Sorption Experiments of Toluene and 8883 Sample at $60{ }^{\circ} \mathrm{C}$ 


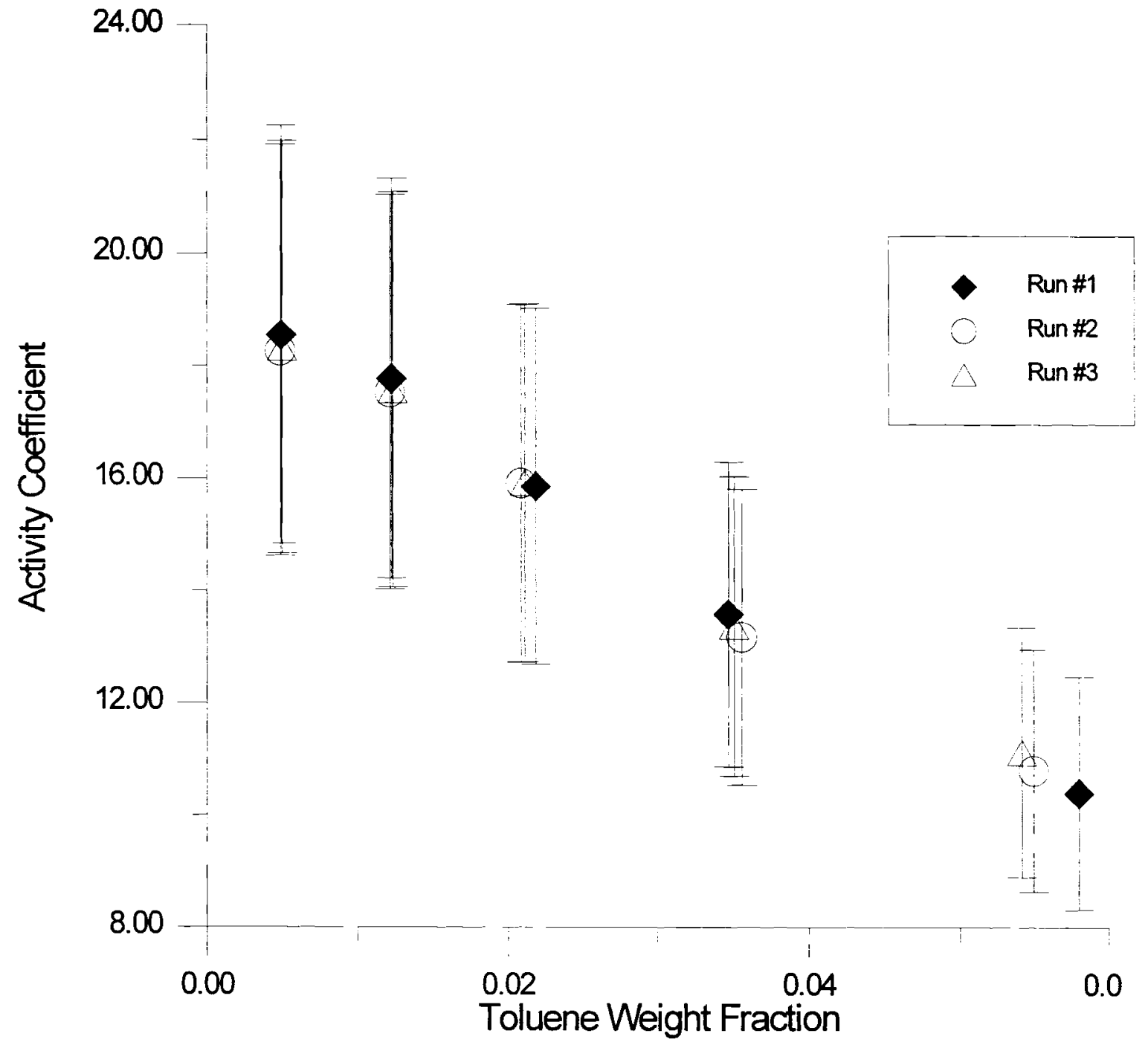

Figure 18.

Equilibrium Sorption Experiments of Toluene and 8883 Sample at $80^{\circ} \mathrm{C}$ 


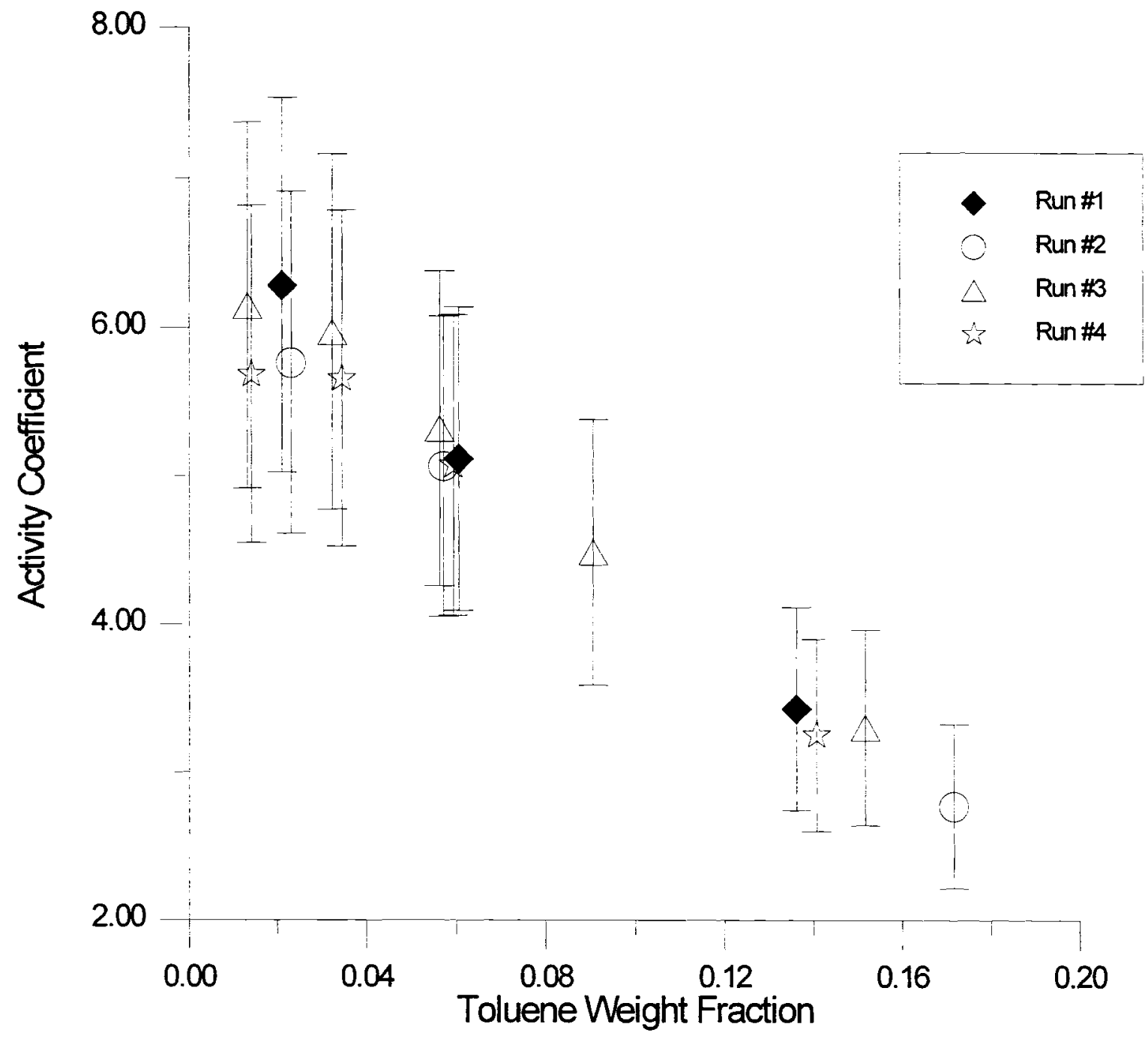

Figure 19.

Equilibrium Sorption Experiments of Toluene and 8899 Sample at $60{ }^{\circ} \mathrm{C}$ 


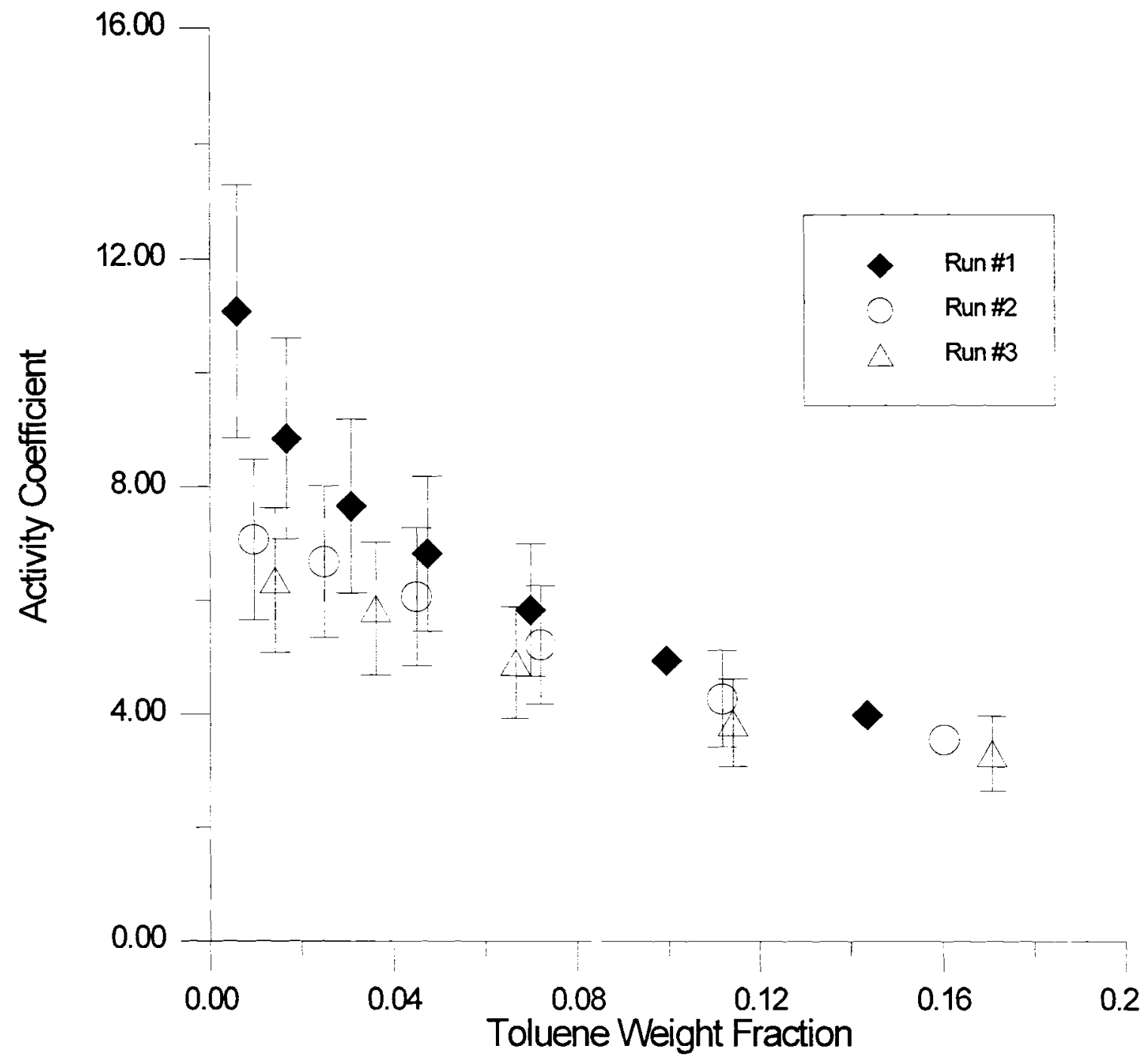

Figure 20.

Equilibrium Sorption Experiments of Toluene and 8899 Sample at $80{ }^{\circ} \mathrm{C}$ 
copolymer. The sample is a proprietary copolymer of ethylene and propylene. Abnormal behavior was observed when the crystals were coated with the copolymer. Normally the frequency of the crystal decreases after coating the crystal with the polymer and while performing the sorption experiments (Mikkilineni et al., 1995). However, as can be seen from Figure 21, the frequency of the coated crystal was highly unstable. The data were taken while desorbing the polymer in order to find the frequency of the crystal and the polymer film. The qualitative behavior of the crystal in Figure 21 was in contrast with the normal crystal behavior. The normal crystal behavior while desorbing the polymer can be obtained from Mikkilineni et al.(1995).

Frequency data were also recorded to check for the frequency characteristics of the coated crystal after the solvent is introduced into the chamber. The data are shown in Figure 22. As can be seen in the Figure 22, the crystal behavior was in contrast to the expected behavior. The frequency of the crystal increased upon introduction of the solvent into the chamber. But normally the frequency of the crystal decreases as the solvent diffuses into the polymer. A constant value of $5971000 \mathrm{~Hz}$ was subtracted from all the frequency data (Y-axis) in Figures 21 and 22 for ease of plotting.

The cause for the abnormal behavior of the crystal is not clearly understood. Some of the probable causes for the abnormal behavior are as follows:

1. The coated polymer sample is highly viscous and there is a possible loss of energy due to viscous dissipation in the sample. As a result of this highly viscous loading on the crystal, the crystal oscillations could have been damped. 


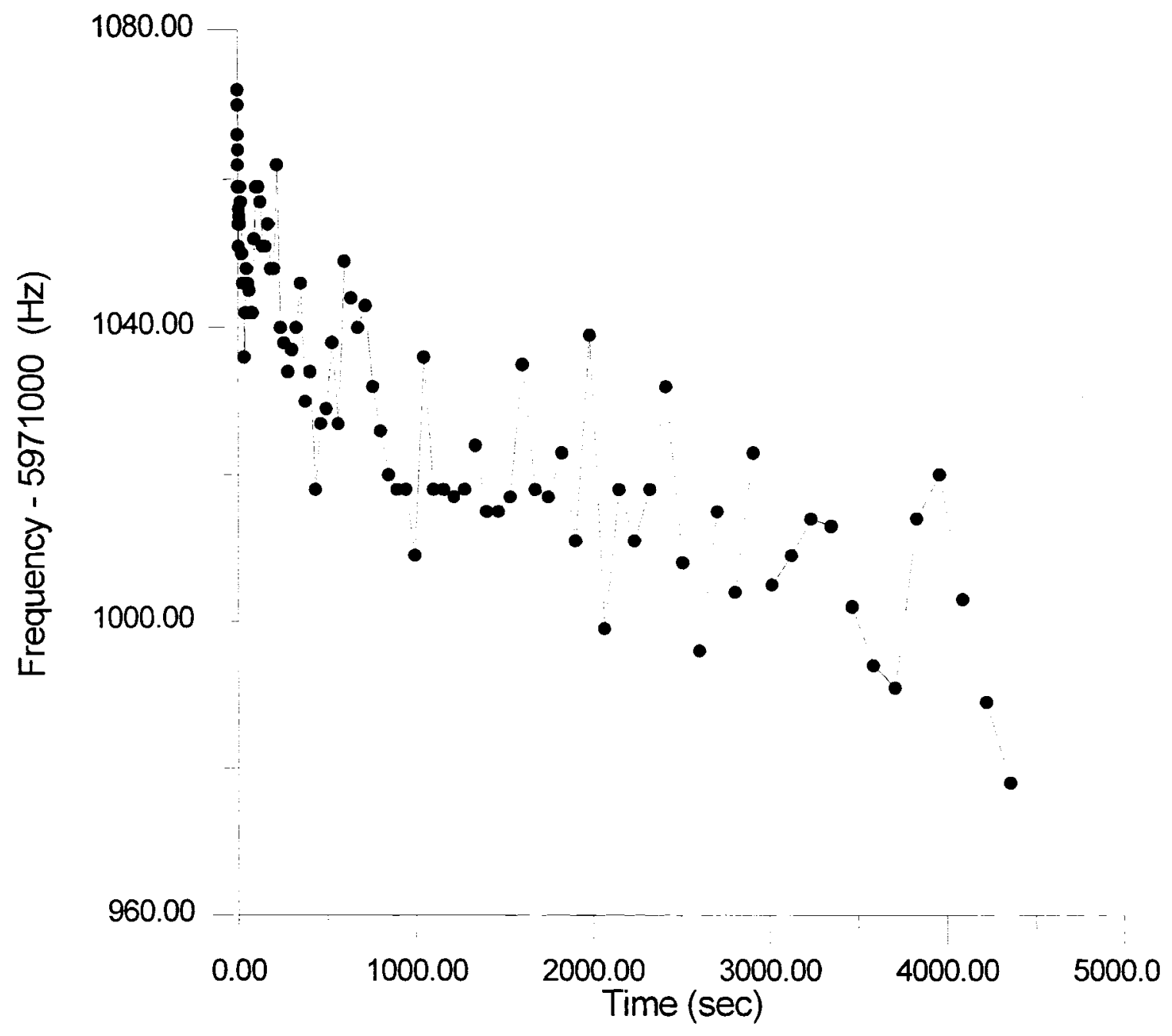

Figure 21 .

Frequency vs Time During Desorption of the Polymer Sample in order to Determine the Frequency of the Polymer and the Crystal 


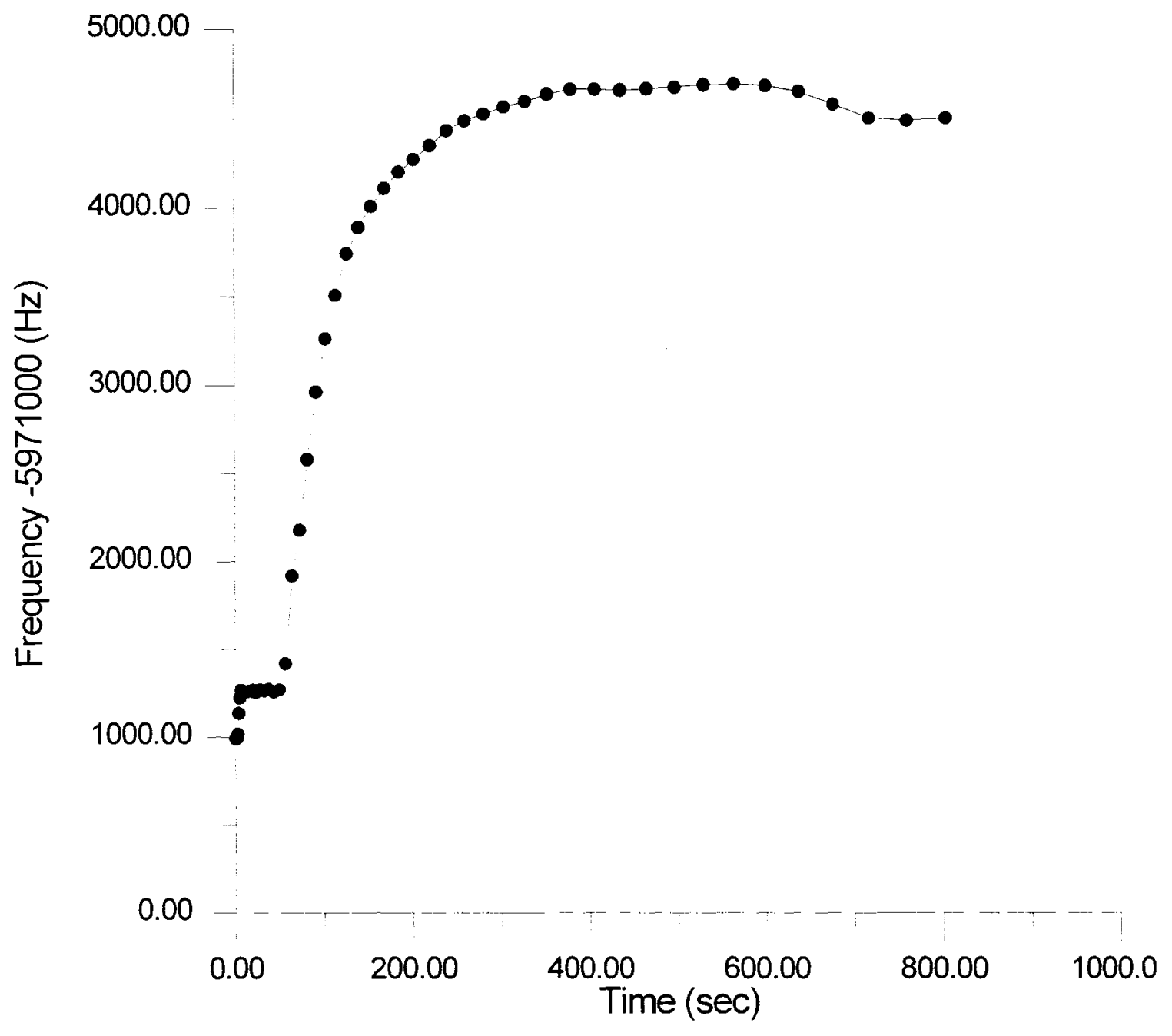

Figure 22.

Frequency vs Time after the Introduction of Solvent into the Chamber 
2. The crystal might be oscillating beyond the detection range of the oscillator circuit used in the $\mathrm{XTC} / 2$ monitor. One solution to this problem is to build an oscillator circuit which has a very strong feedback signal to guarantee the oscillation of even heavily damped crystals.

3. The crystal might have been experiencing stress effects due to the polymer coating (Melroy, 1995). More detailed description of the stress effects can be obtained from Chapter II.

The viscoelastic properties of the polymer film can damp the oscillations of the quartz crystals. This could happen if the shear wave velocity in the polymer film is very low or if the film has a low elastic shear modulus. If the film thickness is an odd multiple of a quarter wavelength of shear waves, the film resonates and the losses in the compound (quartz/polymer) resonator get very large, making it impossible for the crystal to oscillate. If the film thickness is between one quarter and three quarter wavelengths, it is possible for the resonant frequency of the compound resonator to be larger than the bare crystal frequency. As one approaches film thicknesses of one quarter wave length, the frequency of the oscillator begins to reduce rapidly and the oscillations cease. As the thickness of films is increased, the oscillator resumes oscillations at a higher frequency. The operation of crystals under viscoelastic loading is not yet fully understood and more study needs to be done in this area (Kanazawa, 1995). 
An informative experiment suggested by Kanazawa (1995) is to measure the electrical characteristics of the compound (quartz/polymer) resonator as a function of thickness. These experiments should be performed with extremely thin films. The frequency at which the resistance due to the polymeric film is a minimum and the value of the resistance can be evaluated from these experiments. Kanazawa (1995) suggested that the resistance due to the polymer film would probably be constant at low thicknesses. He also suggested that the resistance would probably increase rapidly (or resonant frequency would reduce rapidly) as the thickness of the film approaches one quarter wavelength of the shear waves. The film thicknesses for which the oscillator technique is no longer valid can be evaluated from these experiments (Kanazawa, 1995).

If a viscoelastic polymer is coated on the crystal, appreciable acoustic phase shift occurs in the film and the upper regions of the film lag behind the crystal/film interface. Significant shear deformation occurs in the film causing storage and dissipation of elastic energy in the polymer film. Typically, if a film is bonded to the resonator surface sufficiently well, the base of the film moves synchronously with the resonator surface. The motion in the remainder of the film depends upon the operating frequency as well as the film's mass density, shear modulus and thickness. For frequencies of several megahertz and films of a few microns thickness, the acoustic phase shift occurring across the film, $\phi$, is much less than $\pi / 2$ for glassy films. The phase shift can be of the order of $\pi / 2$ for rubbery films. If the film coating is rigid, the acoustic phase shift is very small and the film moves synchronously with the resonator surface. If the film is rigid, no significant power dissipation occurs in the film and the resonator remains undamped. 
For $0<\phi<\pi / 2$, displacement at the upper surface of the film exceeds the displacement at the resonator surface. As $\phi$ approaches $\pi / 2$ the film starts to oscillate (film resonance) and the compound resonator [film + crystal] exhibits the characteristics of coupled resonator systems. The displacement in the film and resonator exhibits inphase and out-of-phase modes with a concurrent splitting of resonance frequencies. For $\phi$ $=\pi / 2^{-}$(slightly less than $90^{\circ}$ ) the film displacement is large and in phase with the displacement of the resonator surface. In this case the frequency of the coated crystal is lower than the frequency of the uncoated crystal. For $\phi=\pi / 2^{+}$(slightly greater than $90^{\circ}$ ) the film surface is $180^{\circ}$ out of phase with the resonator surface and in this the frequency of the coated crystal is higher than that of the bare crystal, and the system is highly damped in the vicinity of resonance. The resonant frequencies and damping depends upon the shear elastic properties of the film, film thickness, and the density of the material (Martin and Frye, 1991).

When it is difficult to observe the dynamic behavior of the film, the influence of the film properties on the resonator behavior can be determined by monitoring the electrical properties of the compound resonators (Martin and Frye, 1991). Additional description of quartz crystals loaded with viscoelastic substances can be obtained from Chapter III of this thesis. 


\section{CHAPTER V}

\section{CONCLUSIONS}

1. The QCM was proven to be a powerful experimental tool to measure the thermophysical properties of polymer solutions. Experiments can be performed with the QCM to measure equilibrium sorption and diffusivity data in a short time. The time taken to conduct the experiments on QCM is much lesser than the time taken with gravimetric sorption techniques. The QCM can be easily interfaced with a computer, making the data collection fast, easy and accurate.

2. The QCM was also proven to be a suitable tool for industrial applications. Experiments were performed with industrial polymer coatings. While experiments failed with the highly viscous polymers (Case Study II), favorable results were obtained for other industrial polymer coatings (Case Study I).

3. The diffusion coefficients obtained from the QCM were in good agreement with results obtained from other experimental techniques, and were also in good agreement with the predictions of the free volume models. The equilibrium sorption results obtained from QCM were in good agreement with the OishiPrasunitz model, and were in qualitative agreement with the results from other experimental techniques. 
4. Some of the disadvantages of using the QCM with highly viscous polymers have been encountered. QCM experiments could not be performed with the viscous industrial polymers. Crystals ceased to oscillate when coated with the polymer. The exact reason for the crystal failure is not clearly understood.

5. More detailed study needs to be done to understand the behavior of QCM under viscous loading. Very little work has been done in studying the oscillation characteristics of viscoelastically loaded crystals.

6. The agreement of QCM data (within error limits of $\pm 20 \%$ ) with the predictions of Free Volume theory indicate that the diffusion of penetrants in polymers is dependent upon the amount of free volume present in the polymer. 


\section{CHAPTER VI}

\section{RECOMMENDATIONS}

The following recommendations can be made based on the experiments performed on the QCM with various polymers.

1. A better method to determine the thickness of the coated film should be developed. In this study, a known weight of polymer is dissolved in a known volume of solvent. The crystal is then coated by dropping a known volume of the solution onto the crystal with a micro pipette. The thickness of the film is calculated from the volume of the solution used to coat the crystal and the amount of dilution. The thickness of the coated films was not uniformly deposited on the surface of the crystals. There are errors associated with volume measurements of the micropipette. These errors can propagate into the thickness calculations and also affect our diffusion coefficients. A new method such as profilometry should be used to calculate the thickness of the polymer films accurately.

2. A better method to coat the crystals with highly viscous polymers should be developed. When coating the crystals with highly viscous polymers, difficulty was encountered in the use of micro pipette to drop a small volume of solution on the crystal surface. This difficulty can be overcome by dissolving the 
polymers in solvents with high boiling points, so the solutions can be heated to reduce the viscosity before coating.

3. The electronics of the $\mathrm{XTC} / 2$ should be modified to detect the frequencies of the crystals coated with highly viscous samples. When the quartz crystals were coated with industrial copolymers from the Dow Chemical Company an abnormal crystal behavior was encountered. This could be due to the damping of the crystals because of high viscous loading on the crystals. This problem could be solved by building a new feedback oscillator with variable feedback providing a sinusoidal signal with constant amplitude to guarantee the oscillation of even heavily damped quartz crystals.

4. More experiments should be performed with highly viscous polymers to understand the behavior of the crystals under viscous loading. As was mentioned earlier, strange crystal behavior was observed when the crystal is coated with certain samples. A detailed study on the behavior of quartz crystals when coated with viscoelastic materials has not done to date. In order to understand the crystal behavior on viscoelastic loading, a systematic study with various polymers with varying properties should be done.

5. Experiments should be performed on the QCM with other polymer-solvent systems. Some of the systems that could be studied are poly(vinyl acetate)-chloroform, poly(vinyl acetate)-ethylbenzene, polystyrene-toluene, polystyreneethylbenzene, polystyrene-tetrahydrofuran, polyisoprene-cyclohexane. These are the systems with widely different diffusion characteristics and have data 
available in the literature. Some of these systems should be studied to see if the QCM can reproduce the data available in the literature for systems with widely different diffusion characteristics. Experiments should be performed with a system at different temperatures to study the effect of the glass transition temperature.

6. Very little work has been done, studying the diffusion characteristics in filled polymeric systems. So, experiments should also be performed with filled polymeric systems. Filled polymeric systems are widely used in industrial applications. A new model should be developed for the diffusion of penetrants in filled polymeric systems.

7. A better method should be developed for the determination of the slope of the reduced sorption curve. It was shown in Appendix B that the diffusion coefficient calculation is most sensitive to selection of points for slope calculation. In this study, the points between which the slope was calculated were selected by visual inspection. An ideal step change in pressure was assumed in this analysis. But an ideal step change in pressure was not achieved with the present apparatus. A few seconds elapsed before the pressure reached the desired value. If the apparatus is modified to get a better step change in pressure, the slope can be calculated by fitting the initial portion of the reduced sorption curve to a straight line. 


\section{BIBLIOGRAPHY}

Benes, E., "Improved Quartz Crystal Microbalance Technique," J. Appl. Phys., 56, 608 (1984).

Bilger, R., and J. Heinze "Application of Electrochemical Quartz Crystal Microbalance to Determine the Stoichiometry of Ion Movement during Doping-Undoping Processes in Polypyrrole," Synthetic Metals, 41-43, 2893 (1991).

Bonner, David C., and Y. Chang, "A New Method for Determination of Equilibrium Sorption of Gases by Polymers at Elevated Temperatures and Pressures," Polymer Letters Edition, 13, 259 (1975).

Bottom, Virgil E., " Introduction to Quartz Crystal Unit Design," Van Nostrand Reinhold Company, (1982).

Bruckenstein, S., and M. Shay, "Experimental Aspects of Use of the Quartz Crystal Microbalance in Solution," Electrochim. Acta, 30, 1295 (1985).

Carey, P. W., and B. R. Kowalski, "Chemical Piezoelectric Sensor and Sensor Array Characterization," Anal. Chem., 58, 1986, 3077 (1986).

Chen, F., Fredenslund, Aa., Rasmussen, P. "Group Contribution Flory Equation of State for Vapor-Liquid Equilibria," Ind. Eng. Chem. Res., 29, 875 (1990).

Crank, J., "The Mathematics of Diffusion," Oxford University Press, (1957).

Crank, J., G. S. Park, "Diffusion in Polymers," Academic Press, (1968).

Danner, R. P., and M. S. High, "Handbook of Polymer Solution Thermodynamics," Design Institute of Physical Property Data, 1993.

Deakin, M. R., and D. A. Buttry, "Electrochemical Applications of the Quartz Crystal Microbalance," Anal. Chem., 61, 1147A (1989).

Deakin, M. R., and H. Byrd "Prussian Blue Coated Quartz Crystal Microbalance as a Detector for Electroinactive Cations in Aqueous Solution," Anal. Chem., 61, 1989, 290 (1989).

Deshpande, S., "Development of a Piezoelectric Quartz Crystal Microbalance for the measurement of Penetrant Sorption into Polymers," MS Thesis; Oklahoma State University: Stillwater, 1993. 
Duda, J. L., "Diffusion in Polymer melts," Section III in Devolatilization of Polymers, J. A. Biesenberger, editor, Hanser Punblishers, Munich, 1983.

EerNisse, E. P., "Simultaneous Thin-Film Stress and Mass-Change Measurements Using Quartz Resonators," J. Appl. Phys., 43, 1330 (1972).

Fischer, W. F., and W. H. King Jr., "A New Method for Measuring the Oxidation Stability of Elastomers," Anal. Chem., 39, 1265 (1967).

Hadj-Romdhane, I., "Polymer-Solvent Diffusion and Equilibrium Parameters by Inverse Gas-Liquid Chromatography," Ph.D. Thesis, The Pennsylvania State University (1994).

Hafner, E., "Crystal Resonators," IEEE Transactions on Sonic and Ultrasonics, su-21, 220 (1974).

Hammer, D., E. Benes, and H. K. Pulker, "A Digital Quartz Deposition monitor using a Microprocessor," Thin Solid Films, 32, 47 (1976).

Heising, R. A., "Quartz Crystals for Electrical Circuits," Van Nostrand Company, 1947.

High, M. S., R. P. Danner, "Application of Group Contribution Lattice-Fluid EOS to Polymer Solutions," AIChe Journal, 36, 1625 (1990).

Hlavay, J., and G. G. Guilbault, "Applications of the Piezoelectric Crystal Detector in Analytical Chemistry," Anal. Chem., 49, 1890 (1977).

Hou, A. -C. "Molecular Diffusion in Concentrated Polymer Solutions," Ph.D. Thesis, The Pennsylvania State University (1986).

Ju, S. T., "Oscillatory and Transient Sorption Studies of Diffusion in Polyvinyl Acetate," Ph.D. Thesis, The Pennsylvania State University (1981).

Kanazawa, K. K., "The Physical Description of Films Coating Quartz Resonators," Thin Solid Films, 187, L7 (1990).

Kanazawa, K. K., and O. R. Melroy, "The Quartz Resonator: Electrochemical Applications," IBMJ. Res. Develop., 37, 157 (1993).

Kanazawa, K. K., Almaden Research Center (IBM), Personal Communication (1995).

Karasek, F. W., and K. R. Gibbins, "A Gas Chromatograph Based on the Piezoelectric Detector," Journal of Chromatographic Science, 9, 535 (1971). 
Karasek, F. W., and J. M. Tiernay, "Analytical performance of the Piezoelectric Crystal Detector," Journal of Chromatography, 89, 31 (1974).

Karasek, F. W., P. Guy, H. H. Hill Jr., and J. M. Tiernay, "Chromatographic Design and Temperature-Related Characteristics of the Piezoelectric Detector, "Journal of Chromatography, 124, 179 (1976).

King Jr., W. H., "Piezoelectric Sorption Detector," Anal. Chem., 36, 1735 (1964).

King Jr., W. H., "Coated Piezoelectric Analyzers," US patent 3,164,004, Jan 5, 1965.

King Jr., W. H., C. T. Camilli, and C. T. Findeis, "Thin Film Thermocouples for Differential Thermal Analysis," Anal. Chem., 40, 1330 (1968).

King Jr., W. H., and L. W. Corbett, "Relative Oxygen Absorption and Volatility Properties of Submicron of Asphalt using the Quartz Crystal Microbalance," Anal. Chem., 41, 580 (1969).

King Jr., W. H., "Using Quartz Crystals as Sorption Detectors...part 1," Research/Development, 28 (1969a).

King Jr., W. H., "Using Quartz Crystals as Sorption Detectors...part 2," Research/Development, 28 (1969b).

Kinsler, L. E., and A. R. Frey, "Fundamentals of Acoustics," John Wiley \& Sons, 1962.

Klavetter, E. A., S. J. Martin, and K. O. Wessendorf, "Monitoring Jet Fuel Thermal Stability Using a Quartz Crystal Microbalance," Energy and Fuels, 7, 582 (1993).

Konash, P. L., and G. J. Bastiaans, "Piezoelectric Crystals as Detectors in Liquid Chromatography," Anal. Chem., 52, 1929(1980).

Kurosawa, S., E. Nemoto, M. Muratsugu, M. Yoshimoto, Y. Mori, and N. kamo, "Detection of Actomyosin Depolymerization with a Piezoelectric Quartz Crystal," Analytica Chimica Acta, 289, 307 (1990).

Laatikainen, M., and M. Lindstrom, "Measurement of Sorption in Polymer Membranes with a Quartz Crystal Microbalance," Journal of Membrane Science, 29, 127 (1986).

Lee, W., H. S. White, and M. D. Ward, "Depletion Layer Effects on the Response of the Electrochemical Quartz Crystal Microbalance," Anal. Chem., 65, 3232 (1993).

Lin, Z., C. M. Yip, I. S. Joseph, and M. D. Ward, "Operation of an Ultrasensitive 30MHz Quartz Crystal Microbalance in Liquids," Anal. Chem., 65, 1546 (1993). 
Lu, Chih-shun, "Mass Determination with Piezoelectric Quartz Crystal Resonators, " $J$. Vac. Sci. Technol., 12, 578 (1975).

Lu, C., and O. Lewis, "Investigation of Film-thickness Determination by Oscillating Quartz Resonators with Large Mass Load," J. Appl. Phys., 43, 4385 (1972).

Lu, C., Czanderna, A. W., "Applications of Piezoelectric Quartz Crystal Microbalance," Elsevier Science Publishers, 1984.

Martin, B. A., and H. E. Hager, "Velocity Profile on Quartz Crystals Oscillating in Liquids," J. Appl. Phys., 65, 2630 (1989).

Martin, S. J., and G. C. Frye, "Polymer Film Characterization Using Quartz Resoantors," Ultrasonics Symposium, 393 (1991).

McKeown, D., "New Method for Measuring Sputtering Near Threshold," Rev. of Sci. Instruments, 32, 133 (1961).

Mecea, V., and R. V. Bucur, "The Use of RF Voltage in Quartz Crystal Microbalance Measurements: Application to Nonmetallic Films," J. Phys. E., 7, 348 (1974).

Mecea, V., and R. V. Bucur, "The Mechanism of the Interaction of Thin Films with Resonating Quartz Crystal Substrates: The Energy Transfer Model," Thin Solid Films, 60, 73 (1979).

Mecea, V., R. V. Bucur, and E. Indrea, "On the Possibility of Thin Film Structure Study with a Quartz Crystal Microbalance," Thin Solid Films, 171, 367 (1989).

Mecea, V., "Reply to Physical Description of Films Coating Quartz Resonators, by K. K. Kanazawa," Thin Solid Films, 187, L11 (1990).

Melroy, O., Almaden Research Center (IBM), Personal Communication (1995).

Mikkilineni, S. P. V. N., Martin S. High, and D. A. Tree, "Thermophysical Properties of Penetrants in Polymers via a Piezoelectric Quartz Crystal Microbalance," Journal of Chemical and Engineering Data, 40, 750 (1995).

Miller, J. G., and D. I. Bolef, "Acoustic Wave Analysis of the Operation of Quartz Crystal Film-Thickness Monitors," J. Appl. Phys., 39, 5815 (1968).

Moylan, C. R., M. E. Best, and M. Ree, "Solubility of Water in Polyimides: Quartz Crystal Microbalance Measurements", Journal of Polymer Science: Part B: Polymer Physics, 29, 87 (1991). 
Muratsugu, M., F. Ohta, O. Miya, T. Hosokawa, S. Kurosawa, N. Kamo and H. Ikeda, "Quartz Crystal Microbalance for the Detection of Microgram Quantities of Human Serum Albumin: Relationship Between the Frequency Change and Mass of Protein Adsorbed," Anal. Chem., 65, 2933 (1993).

Noël, Marc A. M., and Patrice A. Topart, "High-Frequency Impedance Analysis of Quartz Crystal Microbalances. 1. General Considerations," Anal. Chem., 66, 484 (1994).

Nomura, T., and M. Okuhara, "Frequency Shifts of Piezoelectric Quartz Crystals Immersed in Organic Liquids," Anal. Chim. Acta, 142, 281 (1982).

Oishi, T., J. M. Prausnitz, "Estimation of Solvent Activities in Polymer Solutions Using a Group Contribution Method," Ind. Eng. Chem. Process Des. Dev., 17, 333 (1978).

Panjan, P., A. Zabkar, and B. Navinsek, "Determination of the Interface Width of Thin Multilayered Structures Using Quartz Oscillator Microbalance Depth Profiling," Thin Solid Films, 265 (1989).

Pulker, H. K., E. Benes, D. Hammer, and E. Sollner, "Progress in Monitoring Thin Film Thickness with Quartz Crystal Resonators," Thin Solid Films, 27 (1975).

Quist. A. P., T. Huth-Fehre, and B. U. R. Sundqvist, "Total Yield Measurements in Matrix-assisted Laser Desorption Using a Quartz Crystal Microbalance," Rapid Communications in Mass Spectrometry, 8, 149 (1994).

Redepenning, J., T. K. Schlesinger, E. J. Mechalke, D. A. Puleo, and D. R. Bizios "Osteoblast Attachment Monitored with a Quartz Crystal Microbalance", Anal. Chem., 65, 3378(1993).

Reed, C. E., K. K. Kanazawa, and J. H. Kaufman, "Physical Description of a Viscoelastically Loaded AT-cut Quartz Resonator," J. Appl. Phys., 68, 1993 (1990).

Sauerbrey, G. Z., Phys. Verhandl., 8, 113 (1957).

Sauerbrey, G. Z., Z. Phys., 115, 206 (1959).

Scheide, E. P., G. G. Guilbalt, "Piezoelectric Detectors for Organophosophorus Compounds and Pesticides," Anal. Chem., 44, 1764 (1972).

Schulz, W. W., and W. H. King Jr., "A Universal Mass Detector for Liquid Chromatography," Journal of Chromatographic Science, 11, 343 (1973).

Stockbridge, C. D., "Vacuum Microbalance Techniques," Plenum Press, Vol. 5 (1966). 
Tanaka, S., Y. Iwasaki, K. Ishihara, and N. Nakabayashi "Assessment of Adsorption of Liposomes on a Phospholipid Polymer Surface Using a Quartz Crystal Microbalance," Macromol. Rapid Commun., 15, 1994, 319 (1994).

Utada, T., N. Sugiura, R. Nakamura, and H. Niiyama, "Diffusion Coefficient in Heteropoly Acid Catalyst Measured by a Piezoelectric Quartz Crystal Microbalance," Journal of Chemical Engineering of Japan, 4, 417 (1991).

Vrentas, J. S., C. M. Jarzebski and J. L. Duda, "A Deborah Number for Diffusion in Polymer-Solvent Systems," AIChE Journal, 21, 894 (1975).

Vrentas, J. S., and J. L. Duda, "Diffusion in Polymer-Solvent Systems III, Construction of Deborah Number Diagrams," J. Polym. Sci. Polym. Phys. Ed., 15, 441 (1977).

Vrentas, J. S., J. L. Duda, Y. C. Ni, "Analysis of Step Change Sorption Experiments," J. Polym. Sci. Polym. Phys. Ed., 15, 2039 (1977).

Vrentas, J. S., and J. L. Duda, "Molecular Diffusion in Polymer Solutions," AIChE Journal, 25, 1 (1979).

Vrentas, J. S., and J. L. Duda, "Diffusion," Encyclopedia of Polymer Science and Engineering, Vol. 5 (1986).

Wanzhi, W., H. Changwen, Z. Wenhong, and Y. Shouzhou "A Selective Pharmaceutical Analysis Technique with Sensitive Piezoelectric Crystal Quartz Sensors," Analytical Letters, 26, 2371 (1993).

Ward, M. D., and D. A. Buttry, "In Situ Interfacial Mass detection with Piezoelectric Transducers," Science, 249, 1000 (1990).

Wimmer, L., H. S., J. Hemetsberger, and E. Benes, "New method of Measuring Vibration Amplitudes of Quartz Crystals," Rev. Sci. Instrum., 55, 605 (1984).

$\mathrm{Xu}, \mathrm{H}$., and J. B Schenoff, "Kinetics, Isotherms, and Competition in Polymer Adsorption using a Quartz Crystal Microbalance," Langmuir, 10, 241 (1994).

Yao, S. Z., and T. A. Zhou, "Dependence of the Oscillation Frequency of a Piezoelectric Crystal on the Physical Parameters of Liquids," Anal. Chim. Acta, 212, 61 (1988).

Yin, Y., and R. E. Collins, "Effect of Morphology of Thin Films on the Damping of Quartz Crystal Oscillators," Thin Solid Films, 257, 139 (1995). 
Zabarnick, S., and R. R. Grinstead, "Studies of Jet Fuel Additives Using the Quartz Crystal Microbalance and Pressure Monitoring at $140^{\circ} \mathrm{C}$," Ind. Eng. Chem. Res., 33, 2771 (1994).

Zelinski, J. M., and J. L. Duda, "Predicting Polymer/Solvent Diffusion Diffusion Coefficients Using Free Volume Theory," AIChe Journal, 38, 405 (1992). 


\section{APPENDIX A}

\section{OPERATING INSTRUCTIONS FOR THE QUARTZ CRYSTAL MICROBALANCE}

\section{Preliminary Checks:}

1. Fill the nitrogen trap with liquid nitrogen to prevent contamination of the penetrant atmosphere in the chamber which can occur due to backstreaming of oil vapors from the rotary vane pump. The liquid nitrogen will also protect the pump from solvent vapors.

2. Check the level and condition of the oil in the vacuum pump. A good indication of poor or low oil is when a vacuum of $10^{-1}$ to $10^{-2}$ torr cannot be achieved in the chamber.

3. Check to make sure that all the three valves A, B, C (Figure 5 ) are closed.

\section{Evacuation Procedure:}

The first step to run the experiment is the evacuation of the chamber which is the accomplished by the carrying out the following procedure. These same steps are to be followed whenever the chamber is to be evacuated.

1. Turn on the rotary vane pump.

2. Open the valve connecting the rotary vane pump to the chamber (Valve A).

3. When the pressure decreases below $10^{-1}$ torr, close valve $\mathrm{A}$.

4. Open valve $B$, the high vacuum gate valve. 
5. Switch on the turbomolecular pump. The turbotronic display will show that the pump is in acceleration mode. After approximately 5 minutes, when the turbomolecular pump has accelerated to its maximum speed it will switch to - 'normal' mode of operation.

6. Once the turbomolecular pump has been switched on, the vacuum will be reduced to below $10^{-3}$ torr which is the minimum that the Pirani gauge can read and hence it will display 'OFF'.

7. Switch on the hot gauge, IG3, and press the 'DEGAS' switch.

8. Allow the evacuation process to proceed for approximately $3-4 \mathrm{hrs}$. This will complete the evacuation process.

9. Since the experiment has to be conducted at constant temperature, the thermal box has to be heated to the required temperature. This is done by switching on the blower and the heating element in the thermal box. The temperature of the box is set to the required temperature. The temperature is then allowed to stabilize for about 12 hrs.

\section{Diffusivity Measurements:}

Three steps are involved in obtaining the diffusion coefficient using the quartz crystal microbalance:

1. Measurement of the frequency of the bare crystal

2. Measurement of the frequency change of the polymer coated crystal.

3. Measurement of the frequency change during the experiment as the solvent is sorbed. 


\section{Measuring the Frequency of the Bare crystal:}

1. The bare crystal should be placed in the crystal holder and mounted in the chamber.

2. The chamber is then evacuated for about $2-3 \mathrm{hrs}$.

3. The frequency of the bare crystal should be recorded with the data acquisition computer until it reaches a steady value.

\section{Measuring the Frequency of the Polymer Coated Crystal:}

1. Carefully remove the crystal holder from the chamber.

2. Coat the crystal with a polymer film of the required dimensions.

3. Place the crystal into the holder and the holder into the chamber again.

4. The chamber should then be evacuated for 3-4 hrs.

5. The frequency of the crystal is recorded once it reaches a steady value. To check if the frequency has reached a steady value, the frequency is recorded for about 15 minutes. The frequencies are recorded in a file by running the program 'XTC5.BAS' on the computer that is connected to the deposition monitor. On running the program the computer asks for a file name to record the data. The computer then asks for the command corresponding to the type of the data required. The command for obtaining the crystal frequency as the output 'S13' is entered. The computer records the data as a function of time. 
Measuring the Change in Frequency of the Crystal as the Penetrant Diffuses into the Polymer Film:

Once the frequencies of the bare and polymer coated crystal are noted, the following steps are carried out to record the change in frequency as a function of time, 1. Switch off the IG3 hot ionization gauge. This is because the penetrant will enter the hot cathode gauge and cause a malfunction.

2. Activate the deposition monitor by pressing the 'START' button.

3. Run the computer program 'XTC5.BAS' and enter the file name as required.

4. Input the command 'S13'.

5. Close the gate valve B.

6. Open and close the penetrant valve to obtain the desired penetrant pressure. Steps 4,5 , 6 have to be carried out as quickly as possible.

7. Switch off the turbomolecular pump.

8. Switch off the rotary vane pump. Allow the experiment to run until the mass of the solvent sorbed reaches a value that remains constant for about 30 minutes.

9. The frequency of the crystal is recorded as a function of time.

10. Repeat steps $6,7,8,9$ until the chamber pressure is close to the vapor pressure of the solvent at the chamber temperature.

\section{To End the Experiment:}

1. Stop recording data on the computer.

2. Switch on the roughing pump. 
3. Evacuate the chamber with liquid nitrogen in the $\mathrm{LN}_{2}$ trap.

\section{Coating Procedure:}

1. Measure the weight of an empty bottle.

2. Measure the weight of bottle with polymer crystals (around 10-12 crystals).

3. Measure weight of the bottle after filling the bottle with a solvent.

4. Wait until the polymer crystals to totally dissolve in the solvent.

5. Take a known volume of the solution (around 5-7 $\mu \mathrm{L}$ ) with the micropipette.

6. Coat the quartz crystal with the solution in the micropipette by dropping the solution onto the crystal surface.

7. Wait until the solvent evaporates from the crystal surface. If needed, insert the crystal in the thermal box. 


\section{APPENDIX B}

\section{ERROR AND SENSITIVITY ANALYSIS}

The aim of this analysis is to calculate the error that has propagated into the final calculated quantity of mutual diffusivity due to uncertainties in the measured quantities. The final quantity calculated is expressed as a function of the measured variables. If we consider $y$ as the calculated quantity, then the uncertainty in this quantity due to the measured variables $\mathrm{x}_{1}, \mathrm{x}_{2}, \ldots . \mathrm{x}_{\mathrm{n}}$ is given by,

$$
\sigma_{y}=\sqrt{\sum_{x=1}^{n}\left[\left(\partial y / \partial_{x i}\right)^{2} \sigma_{x i}^{2}\right]} .
$$

In this analysis, the uncertainty propagated in the diffusion coefficient due to the uncertainties in the measured variables is calculated. The diffusivity is calculated from the measured frequency of the crystal and thickness of the film.

$$
D=\frac{\pi}{4} l^{2} R^{2},
$$

where

$$
\begin{aligned}
& \mathrm{D}=\text { diffusion coefficient }, \\
& \mathrm{l}=\text { thickness of film, } \\
& \mathrm{R}=\left(\frac{\mathrm{d} / \mathrm{dt}(\mathrm{m}(\mathrm{t}) / \mathrm{m}(\infty))}{\sqrt{\mathrm{t}}}\right),
\end{aligned}
$$




$$
\text { or slope of the curve } \frac{m(t)}{m(\infty)} \text { vs } \sqrt{t} \text {. }
$$

So, the error in diffusion coefficient is:

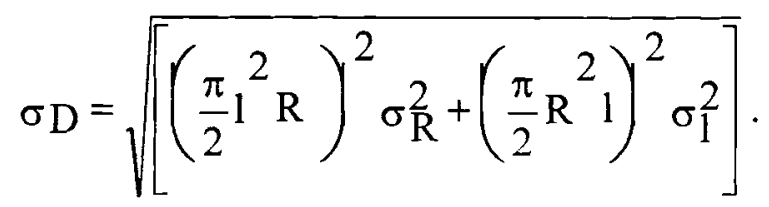

The uncertainties in 1 and $\mathrm{R}$ have to be estimated. The uncertainty in the thickness of the film has been assumed to be $10 \%$. The value of $\mathrm{R}$ is calculated from

$$
\mathrm{R}=\frac{\mathrm{Y}_{2}-\mathrm{Y}_{1}}{\mathrm{X}_{2}-\mathrm{X}_{1}},
$$

where

$$
\begin{aligned}
& Y_{2}=\text { Value of } \mathrm{m}(\mathrm{t}) / \mathrm{m}(\infty) \text { at point } 1, \\
& Y_{1}=\text { Value of } \mathrm{m}(\mathrm{t}) / \mathrm{m}(\infty) \text { at point } 2, \\
& \mathrm{X}_{2}=\text { Value of } \sqrt{\mathrm{t}} \text { at point } 1 \text {, and } \\
& X_{1}=\text { Value of } \sqrt{\mathrm{t}} \text { at point } 2 .
\end{aligned}
$$

Since the values of time are taken from the computer clock, the error in $t$ is assumed to be zero. The quantity $\mathrm{R}$ is simply the slope of the reduced sorption curve. The error associated with $\mathrm{R}$ is:

But

$$
\sigma_{\mathrm{R}}=\sqrt{\left(\frac{\partial \mathrm{R}}{\partial \mathrm{Y}_{2}}\right)^{2}{\sigma_{\mathrm{Y}_{2}}}^{2}+\left(\frac{\partial \mathrm{R}}{\partial \mathrm{Y}_{1}}\right)^{2}{\sigma_{\mathrm{Y}_{1}}}^{2}} .
$$

$\mathrm{f}_{1}=$ Frequency of the coated crystal,

$f_{1}(t)=$ Frequency of the crystal run at any time $t$, and 


$$
f_{1}(\infty)=\text { Frequency of the crystal at the equilibrium }
$$

So the error in $\mathrm{Y}$ is:

$$
\sigma_{\mathrm{Y}_{2}}=\sigma_{\mathrm{Y}_{1}}=\sqrt{\left(\frac{\partial \mathrm{Y}}{\partial \mathrm{f}_{1}(\mathrm{t})}\right)^{2} \sigma_{\mathrm{f}_{1}(\mathrm{t})}^{2}+\left(\frac{\partial \mathrm{Y}}{\partial \mathrm{f}_{1}(\infty)}\right)^{2} \sigma_{\mathrm{f}_{1}(\infty)}^{2}+\left(\frac{\partial \mathrm{Y}}{\partial \mathrm{f}_{1}}\right)^{2} \sigma_{\mathrm{f}_{1}}^{2}}
$$

Here the measured quantities are the frequencies $f_{1(t)}, f_{1(\infty)}, f_{1}$. The errors associated with these variables are assumed as:

$$
\begin{aligned}
& \sigma_{f_{1}(t)}=0.1 \mathrm{~Hz}, \\
& \sigma_{f_{1}(\infty)}=10 \mathrm{~Hz}, \text { and } \\
& \sigma_{f_{1}}=10 \mathrm{~Hz} .
\end{aligned}
$$

The uncertainty associated with $f_{1}(t)$ has been given by the manufacturer. The uncertainties associated with $f_{1}$ and $f_{1}(\infty)$ are based on experimental runs, and is a very conservative estimate of the error.

Evaluation of equation (E-8) gives

$$
\sigma_{\mathrm{Y}}=\sqrt{a^{2} \sigma_{f_{1}(t)}^{2}+b^{2} \sigma_{f_{1}(\infty)}^{2}+c^{2} \sigma_{f_{1}}^{2}}
$$

where

$$
\begin{aligned}
& a=\left(\frac{1}{\left(f_{1}(\infty)-f_{1}\right.}\right) \\
& b=\left(-\frac{\left(f_{1}(t)-f_{1}\right)}{\left(f_{1}(\infty)-f_{1}\right)^{2}}\right) \\
& c=\left(-\frac{\left(f_{1}(\infty)-f_{1}(t)\right)}{\left(f_{1}(\infty)-f_{1}\right)^{2}}\right)
\end{aligned}
$$

$\sigma_{\mathrm{Y}}$ is then evaluated from equation (E-9). The error in the slope is calculated by evaluating $\sigma_{\mathrm{Y}}$ at both the points between which the slope is taken and then using 
$\sigma$ is then evaluated from equation (E-9). The error in the slope is calculated by evaluating $\sigma$ at both the points between which the slope is taken and then using equation (E-6). The error in the diffusion coefficient is obtained from equation (E-4). In the error calculations for diffusion coefficient, the error in film thickness calculation is assumed to be $10 \%$.

\section{SAMPLE CALCULATION:}

A sample calculation is made to demonstrate the error calculations. Similarly errors can be calculated for all the data in the results and discussion chapter.

The following data are used for the sample calculation.

Diffusion Coefficient $=9.94 \times 10^{-10} \mathrm{~cm}^{2} / \mathrm{s}$ (Table II)

Film Thickness $=5.45 \times 10^{-4} \mathrm{~cm}$

Initial slope of the sorption curve $=0.065261$

$f_{1}=5956942 \mathrm{~Hz}$

$f_{1}(\infty)=5955504 \mathrm{~Hz}$

$f_{1}(t)$ at point 1 of slope calculation $=5956432 \mathrm{~Hz}$

$\mathrm{f}_{1}(\mathrm{t})$ at point 2 of slope calculation $=5956197 \mathrm{~Hz}$

Error in $\mathrm{Y}$ calculation at point $1=2.67 \times 10^{-7}$ (from E-9)

Error in $\mathrm{Y}$ calculation at point $2=2.47 \times 10^{-7}$ (from E-9)

Error in Slope $=0.000717$ (from E-6)

Error in Diffusion coefficient $=2 \times 10^{-10}$ (from E-4)

The relative error in the calculation of diffusion coefficient is $20 \%$. 


\section{ERROR IN ACTIVITY COEFFICIENT:}

Activity coefficient is determined by the formula:

$$
\Omega=\frac{\mathrm{a}}{\omega}
$$

where

$$
\begin{aligned}
& a=\text { The activity of the solvent } \\
& \omega=\text { The equilibrium weight fraction of the solvent } \\
& a=\frac{P}{P^{*}} \\
& P=\text { Chamber pressure } \\
& P^{*}=\text { Vapor pressure of the solvent at chamber temperature. } \\
& \omega=\frac{f_{1}(\infty)-f_{1}}{f_{1}(\infty)-f_{0}} \\
& f_{0}=\text { Frequency of the bare crystal }
\end{aligned}
$$

The error in activity coefficient is determined by

$$
\sigma_{\Omega}=\sqrt{\left(\frac{\partial \Omega}{\partial \mathrm{a}}\right)^{2} \sigma_{\mathrm{a}}^{2}+\left(\frac{\partial \Omega}{\partial \omega}\right)^{2} \sigma_{\omega}^{2}}
$$

From equations E-14 and E-1

$$
\sigma_{\mathrm{a}}=\frac{\sigma_{\mathrm{p}}}{\mathrm{P}^{*}}
$$

Here the error in calculation of vapor pressure is assumed to be zero.

From equations E-15 and E-1

$$
\sigma_{\omega}=\sqrt{a^{2} \sigma_{f_{1}}^{2}+b^{2} \sigma_{f_{1}(\infty)}^{2}+c^{2} \sigma_{f_{0}}^{2}}
$$

where 


$$
\begin{aligned}
& a=\left(-\frac{1}{f_{1}(\infty)-f_{0}}\right) \\
& b=\left(\frac{\left(f_{1}-f_{0}\right)}{\left(f_{1}(\infty)-f_{0}\right)^{2}}\right) \\
& c=\left(\frac{f_{1}(\infty)-f_{1}}{\left(f_{1}(\infty)-f_{0}\right)^{2}}\right)
\end{aligned}
$$

From E-13, E-16 and E-1

$$
\sigma_{\Omega}=\sqrt{\left(\frac{\sigma_{\mathrm{p}}}{\omega \mathrm{P}^{*}}\right)^{2}+\left(\frac{\mathrm{a} \sigma_{\omega}}{\omega^{2}}\right)^{2}}
$$

The error in activity coefficient can be calculated from equations E-18 and E-22.

\section{SAMPLE CALCULATION:}

The error in activity coefficient is calculated for the same run for which diffusion coefficient error calculations were done above. The following additional data are used in the error calculations:

Equilibrium weight fraction $=0.054284$

Bare Crystal Frequency $=5981995 \mathrm{~Hz}$

Activity of the solvent $=0.197573$

Activity Coefficient $=3.639626$

Error in equilibrium weight fraction $=0.00052$ (from E-18)

Error in pressure reading $=0.1$ Psia (assumption)

Error in bare crystal frequency $=10 \mathrm{~Hz}$ (assumption)

Vapor pressure of toluene at $60{ }^{\circ} \mathrm{C}=2.6876$ Psia 
Error in activity coefficient $=0.6854$

The relative error in activity coefficient calculation is $18.8 \%$.

\section{SENSITIVITY ANALYSIS:}

Sensitivity analysis has been performed to determine the most sensitive variable in the calculation of diffusion coefficient. The analysis was performed by varying a measured variable by a certain percentage of its original value and keeping all other quantities constant and then observing the resultant change in the diffusion coefficient. This analysis was performed with all the measured quantities used in the calculation of a diffusion coefficient. The sensitivity analysis was performed for a single sample run. The results are as shown below. The different measured quantities used in the calculation of a diffusion coefficient are the thickness of the film, and the frequencies $f_{1}(t), f_{2}(t), f_{1}(0)$ and $f_{1}(\infty)$,

where

$f_{1}(t)=$ Frequency corresponding to the first point in the slope calculation,

$f_{2}(t)=$ Frequency corresponding to the second point in the slope calculation,

$f_{1}(0)=$ Frequency at the start of the sorption run,

$f_{1}(\infty)=$ Frequency at the end of the sorption run. 
Table V

Sensitivity Analysis for Frequency Changes

\begin{tabular}{|c|c|c|c|c|}
\hline $\begin{array}{l}\text { Fractional } \\
\text { Change in } \\
\text { Frequencies } \\
\left(f_{1}(t), f_{2}(t)\right. \\
\left.f_{1}(0), f_{1}(\infty)\right)\end{array}$ & $\begin{array}{l}\text { Fractional } \\
\text { Change in } \\
\text { Diffusion } \\
\text { Coefficient } \\
\text { corresponding } \\
\text { to the change in } \\
\mathrm{f}_{1}(\mathrm{t})\end{array}$ & $\begin{array}{l}\text { Fractional } \\
\text { Change in } \\
\text { Diffusion } \\
\text { Coefficient } \\
\text { corresponding } \\
\text { to the change } \\
\text { in } \mathrm{f}_{2}(\mathrm{t})\end{array}$ & $\begin{array}{l}\text { Fractional } \\
\text { Change in } \\
\text { Diffusion } \\
\text { Coefficient } \\
\text { corresponding } \\
\text { to the change in } \\
\mathrm{f}_{1}(0)\end{array}$ & $\begin{array}{l}\text { Fractional } \\
\text { Change in } \\
\text { Diffusion } \\
\text { Coefficient } \\
\text { corresponding to } \\
\text { the change in } \\
\mathrm{f}_{1}(\infty)\end{array}$ \\
\hline-0.0000001 & $-1.54 \mathrm{E}-2$ & $-2.85 \times 10^{-3}$ & $-7.2 \mathrm{E}-3$ & $-8.85 \times 10^{-3}$ \\
\hline-0.000001 & $-6.00 \times 10^{-2}$ & $4.30 \times 10^{-2}$ & 0.10 & $-1.62 \times 10^{-2}$ \\
\hline-0.00001 & -0.45 & 0.56 & 0.31 & $-8.5 \times 10^{-2}$ \\
\hline-0.0001 & 1.34 & 11.39 & 2.83 & -0.5 \\
\hline 0 & 0 & 0 & 0 & 0 \\
\hline 0.0001 & $-5.40 \times 10^{-3}$ & $-1.29 \times 10^{-2}$ & 0.43 & $-7.20 \times 10^{-3}$ \\
\hline 0.00001 & $4.04 \times 10^{-2}$ & $-5.75 \times 10^{-2}$ & 0.54 & $2.46 \times 10^{-4}$ \\
\hline 0.000001 & 0.55 & -0.45 & -0.17 & 0.08 \\
\hline 0.0000001 & 11.38 & 1.34 & -0.60 & 1.89 \\
\hline
\end{tabular}


In Table $\mathrm{V}$, the $2^{\text {nd }}, 3^{\text {rd }}, 4^{\text {th }}$, and $5^{\text {th }}$ columns are the calculated fractional changes in diffusion coefficients as a result of changes made to the frequencies. One of the frequencies is changed by the fractions shown in the column 1, keeping all other quantities constant and the corresponding changes in the diffusion coefficient values are calculated. These calculations are performed with all the four frequencies $\left(f_{1}(t), f_{2}(t)\right.$, $\left.f_{1}(0), f_{1}(\infty)\right)$ as shown in columns $2,3,4$, and 5 . The fractional changes made to the frequencies are shown in the column 1. From the above table, it can be seen that the diffusivity is most sensitive to the frequencies $f_{1}(t)$ and $f_{2}(t)$. Since $f_{1}(t)$ and $f_{2}(t)$ are used in the calculation of the slope of reduced sorption curve, calculation of slope is very sensitive to changes in these quantities. In this study, the points between which the slope is calculated are selected by visual inspection of the sorption curve. A better method for the calculation of slope should be developed. Slope calculations could be done by fitting the initial part of the reduced sorption curve to a straight line. This was not done in this study because, a perfect step change in pressure was not achieved. It took several seconds for the pressure to build up in the chamber. This resulted in a curved reduced sorption curve in the initial few seconds of the run. A curve fit was not done to avoid the errors due to an improper step change. If the equipment is changed to provide a more ideal step change, the slope can be calculated using straight line fits to the initial portion of the reduced sorption curve. A plot of fractional change in frequencies versus fractional change in diffusion coefficient is shown in Figure 23. 


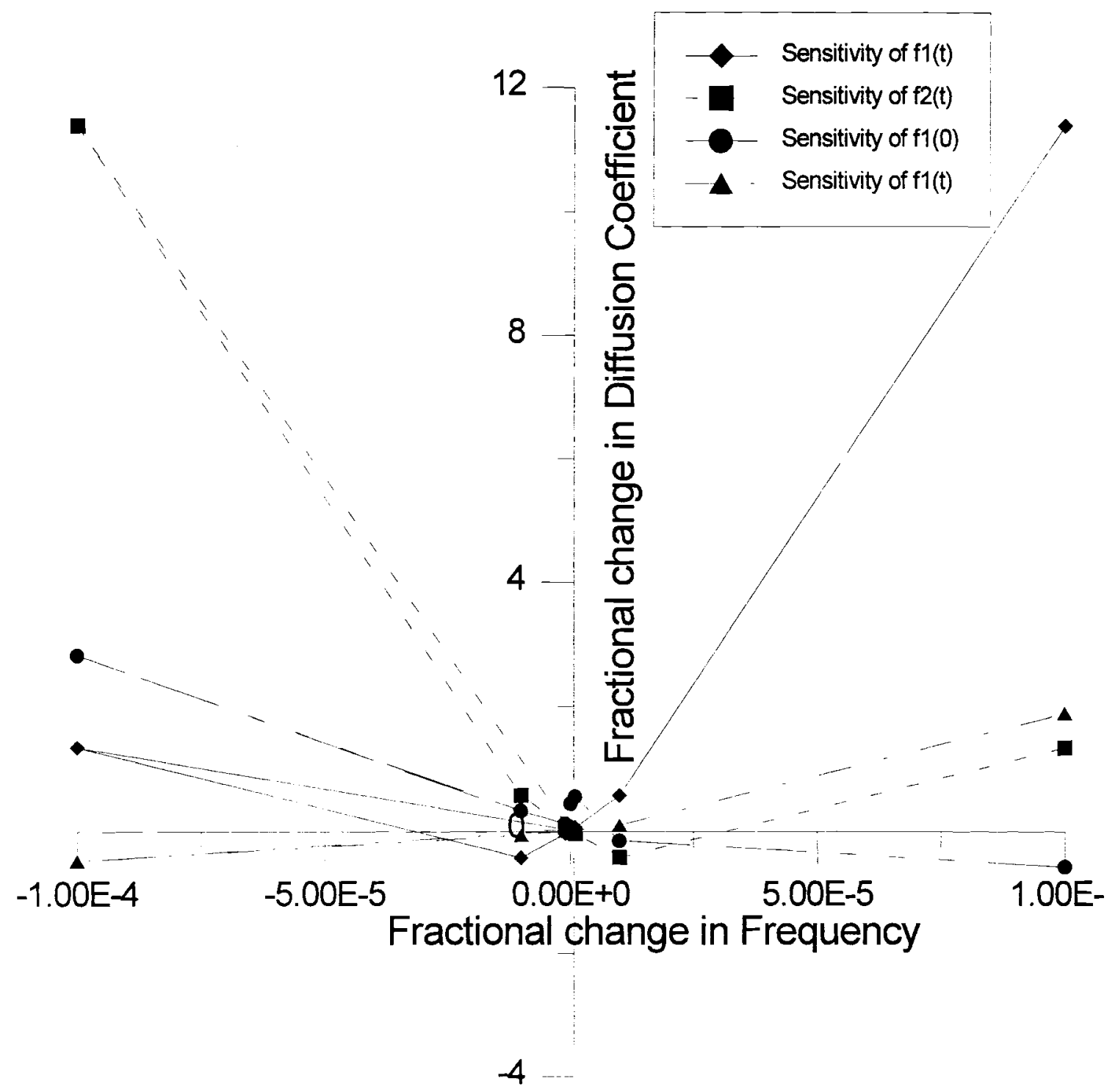

Figure 23.

Sensitivity of Frequencies in the Calculation of Diffusion Coefficient 
Sensitivity analysis was also done with respect to changes in the thickness of the film and the slope of the reduced sorption curve. The results are tabulated in Table VI.

The changes in the thickness of the film and the slope of the reduced sorption curve yielded similar changes to the diffusion coefficient since the formula used for the calculation of diffusion coefficient contains a term involving the product of thickness, $\mathrm{L}$, and slope R. 


\section{Table VI}

Sensitivity Analysis for Changes in Thickness and Slope of the Sorption Curve

\begin{tabular}{lll}
\hline Fractional Change in & Fractional Change in & Fractional Change in \\
Thickness of the Film / & Diffusion Coefficient & Diffusion Coefficient \\
Slope of the Reduced & corresponding to the Change & corresponding to the \\
Sorption Curve & in the Thickness of the Film & Change in the Slope of the \\
& Reduced Sorption Curve
\end{tabular}

\begin{tabular}{lll}
\hline-0.05 & $-9.56 \times 10^{-2}$ & $-9.56 \times 10^{-2}$ \\
-0.1 & -0.19 & -0.19 \\
-0.15 & -0.27 & -0.27 \\
-0.2 & -0.36 & -0.36 \\
-0.25 & -0.44 & -0.44 \\
0 & 0 & 0 \\
0.05 & 0.10 & 0.10 \\
0.1 & 0.21 & 0.21 \\
0.15 & 0.32 & 0.32 \\
0.2 & 0.44 & 0.44 \\
0.25 & 0.56 & 0.56 \\
\hline
\end{tabular}




\title{
VITA
}

\author{
Siva Prasad Mikkilineni \\ Candidate for the Degree of \\ Master of Science
}

Thesis: THERMOPHYSICAL PROPERTIES OF PENETRANTS IN POLYMERS VIA A PIEZOELECTRIC QUARTZ CRYSTAL MICROBALANCE

Major Field: Chemical Engineering

Biographical:

Personal Data: Born in Guntur, India, August 16, 1970, son of Dharmavathi and Surendra Babu Mikkilineni

Education: Graduated from Nirmala High School, Machilipatnam, AP, India, in April 1987; received Bachelor of Engineering Degree in Chemical Engineering and Master of Science Degree in Physics from Birla Institute of Technology and Science in June 1992; completed requirements for the Master of Science degree at Oklahoma State University in December, 1995.

Professional Experience: Employed as a research assistant, School of Chemical Engineering, Oklahoma State University, September 1993 to August 1995. 